\title{
Stereospecific Isomerization of Allylic Halides via Ion Pairs with Induced NonCovalent Chirality
}

Samuel Martinez-Erro, \$ Victor García-Vázquez, \$ Amparo Sanz-Marco, Belén Martín-Matute* belen.martin.matute@su.se

\section{Supporting Information - NMR Spectra}

\section{$\underline{\text { Table of contents }}$}

S2 ${ }^{1} \mathrm{H}$ NMR, ${ }^{13} \mathrm{C}$ NMR and ${ }^{19} \mathrm{~F}$ NMR of allylic halides $\mathbf{2 a - 2 o}, \mathbf{3 a - 3} \mathbf{c}$ and $\mathbf{4 a - 4 c}$

S26 ${ }^{1} \mathrm{H}$ NMR, ${ }^{13} \mathrm{C}$ NMR and ${ }^{19} \mathrm{~F}$ NMR of vinyl halides 5a-5n, 6a-6c and 7a-7c

S45 HPLC Chromatograms of allylic chlorides 2a, 2b, 2c, 2d, 2e, 2m and $2 \mathrm{~g}$

S50 HPLC Chromatograms of vinyl chlorides $5 \mathbf{a}, \mathbf{5 b}, \mathbf{5 c}, \mathbf{5 d}, \mathbf{5 e}, \mathbf{5 m}$ and $\mathbf{5 g}$ 
(E)-(1-chloro-4,4,4-trifluorobut-2-ene-1,3-diyl)dibenzene (2a)

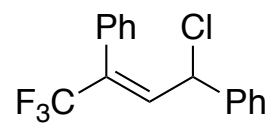
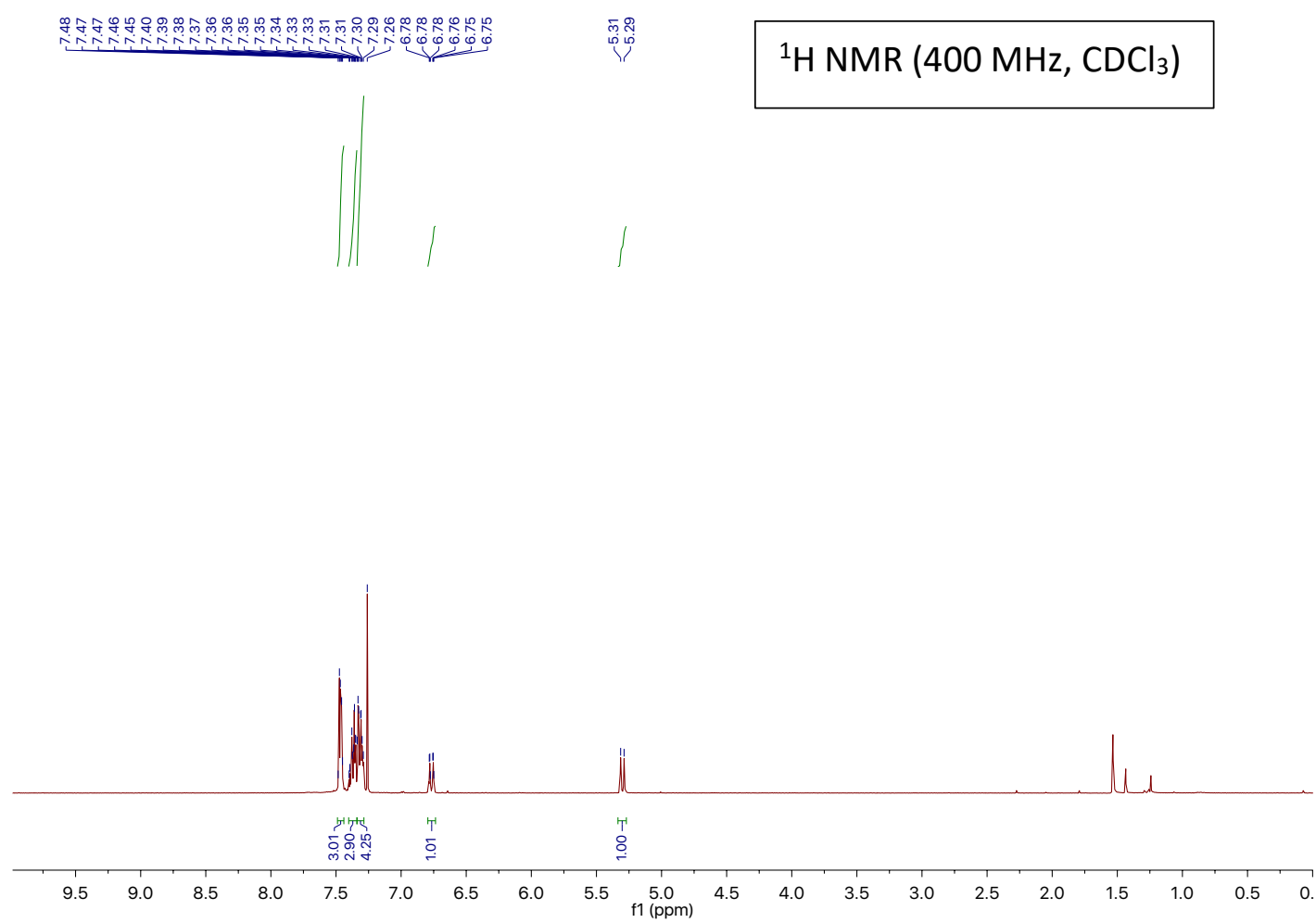

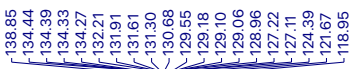
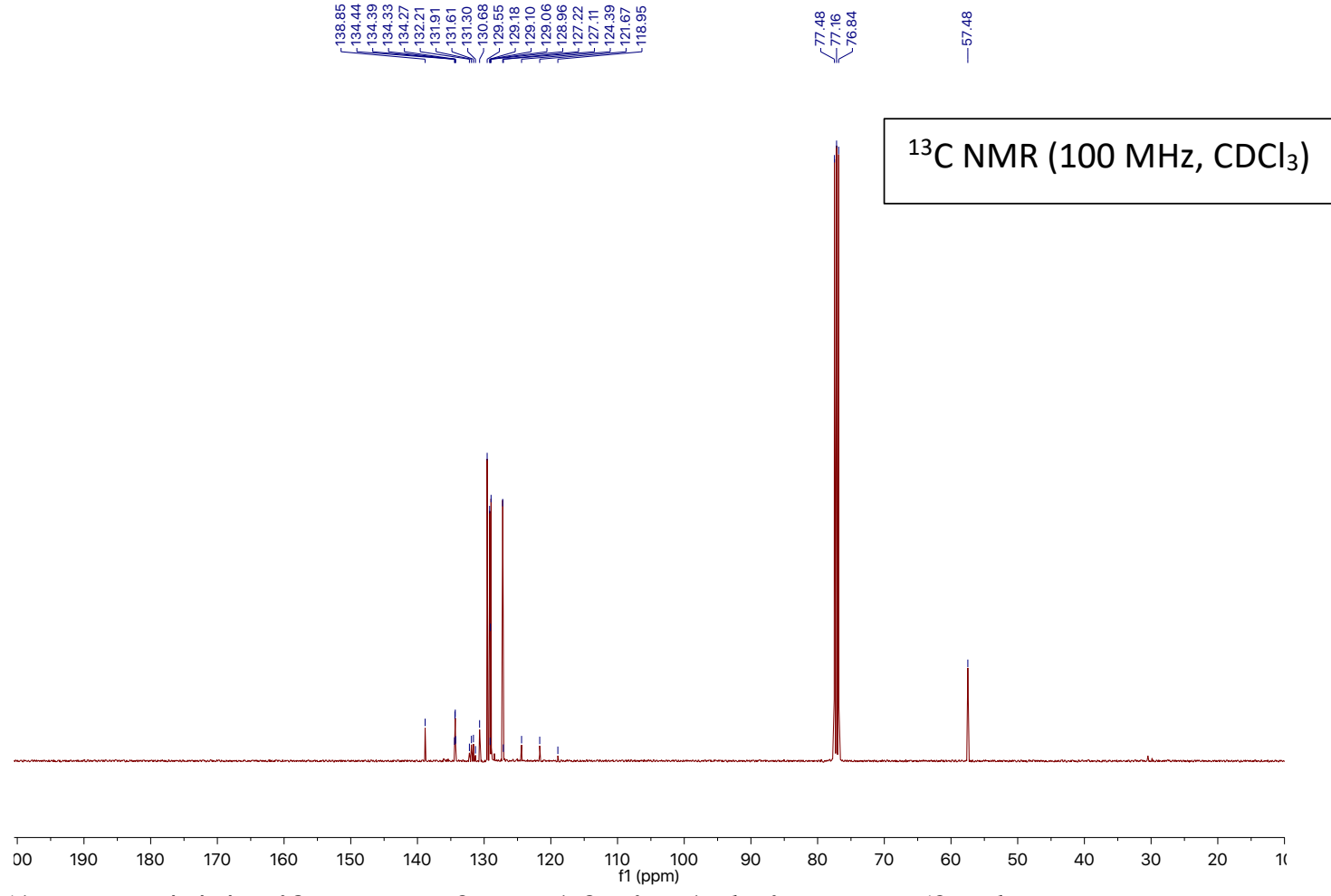

(E)-(1-chloro-4,4,4-trifluorobut-2-ene-1,3-diyl-1- $d$ )dibenzene (2a-d) 

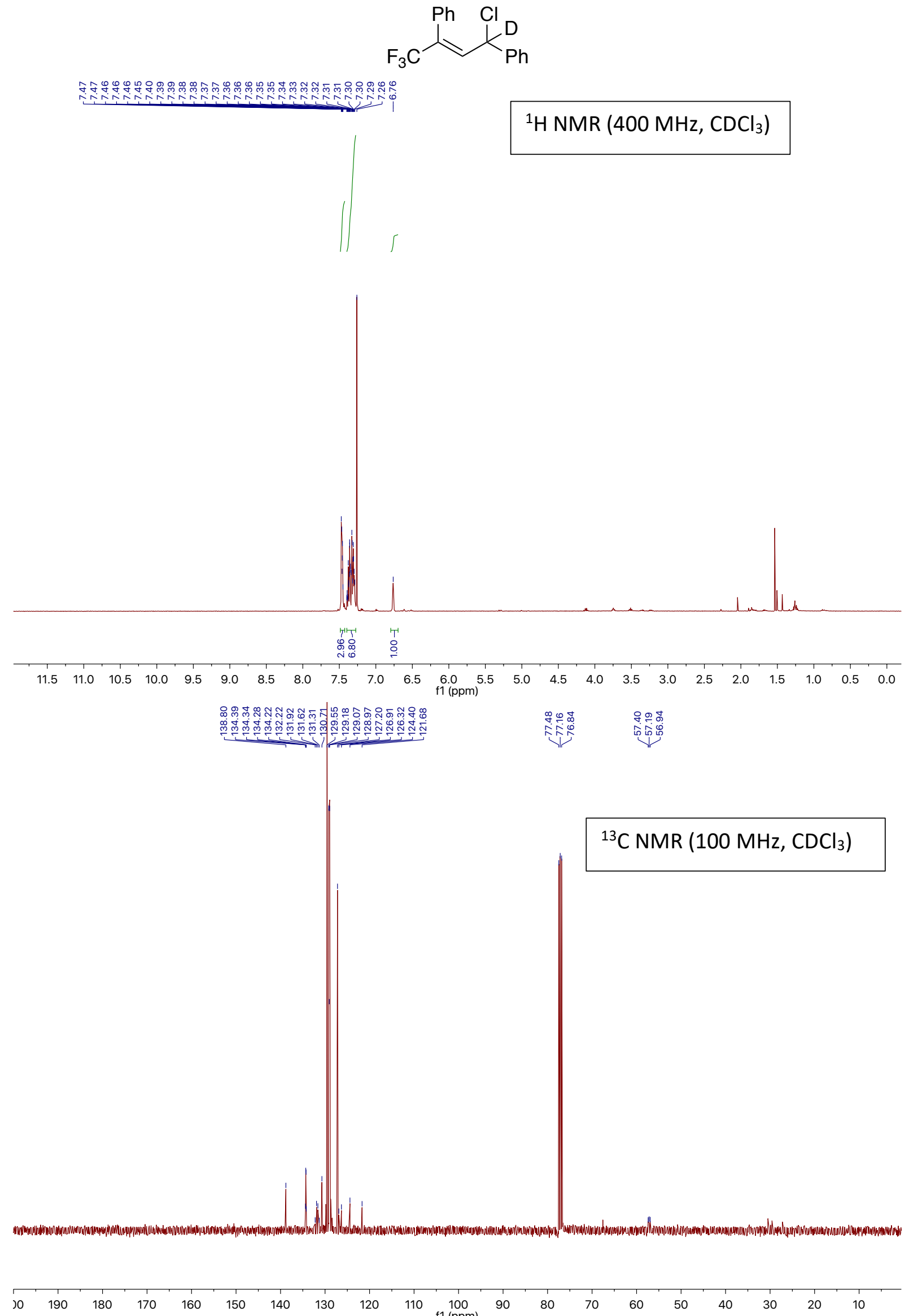
(E)-1-Bromo-4-(1-chloro-4,4,4-trifluoro-3-phenylbut-2-en-1-yl)benzene (2b)
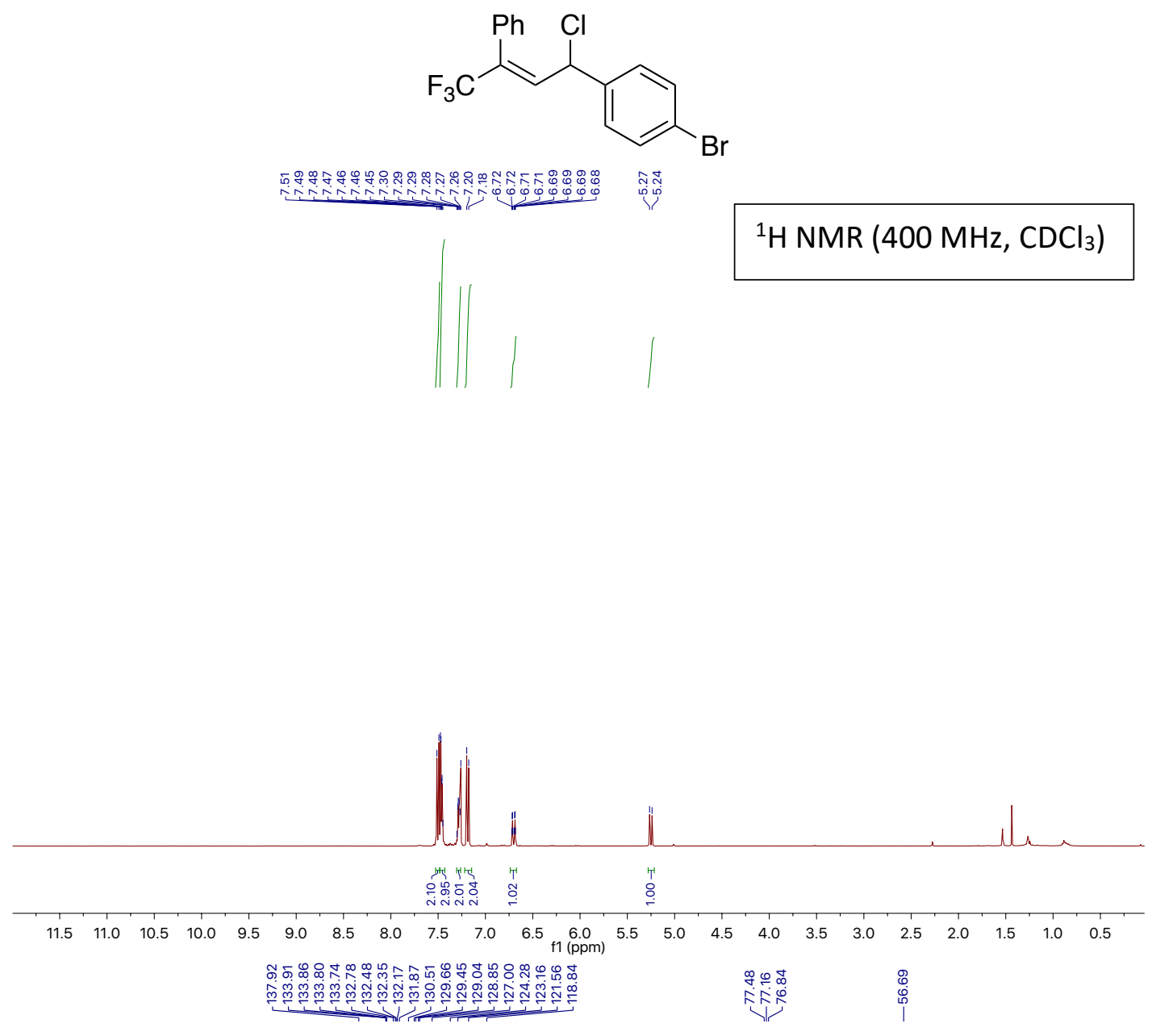

${ }^{13} \mathrm{C}$ NMR $\left(100 \mathrm{MHz}, \mathrm{CDCl}_{3}\right)$

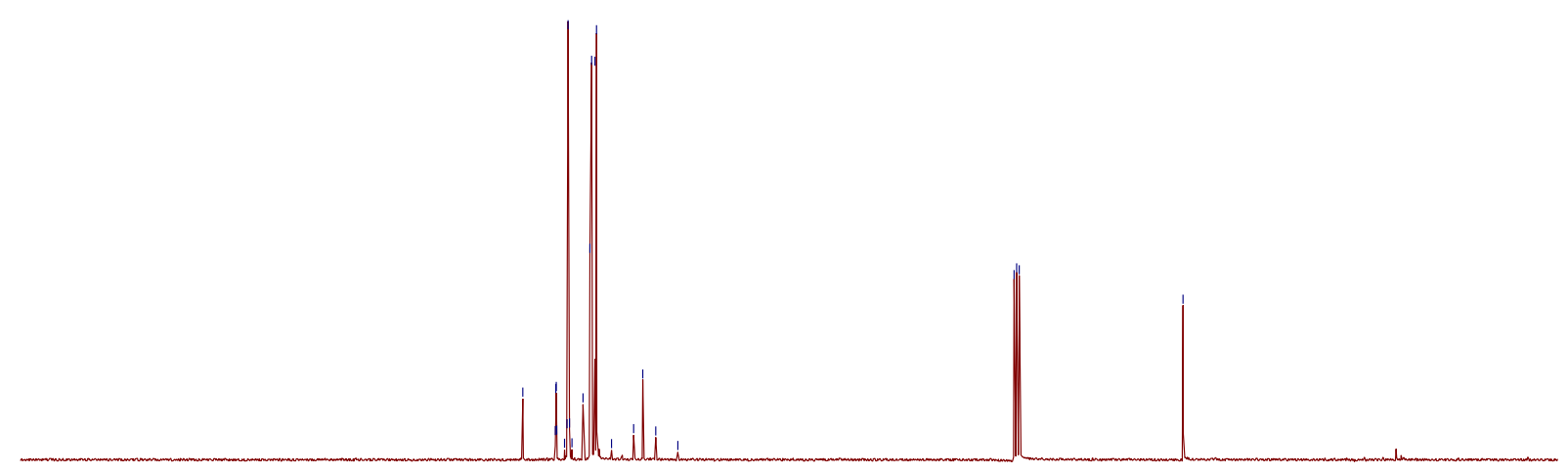


(E)-1-(1-chloro-4,4,4-trifluoro-3-phenylbut-2-en-1-yl)-4-(trifluoromethyl)benzene (2c)

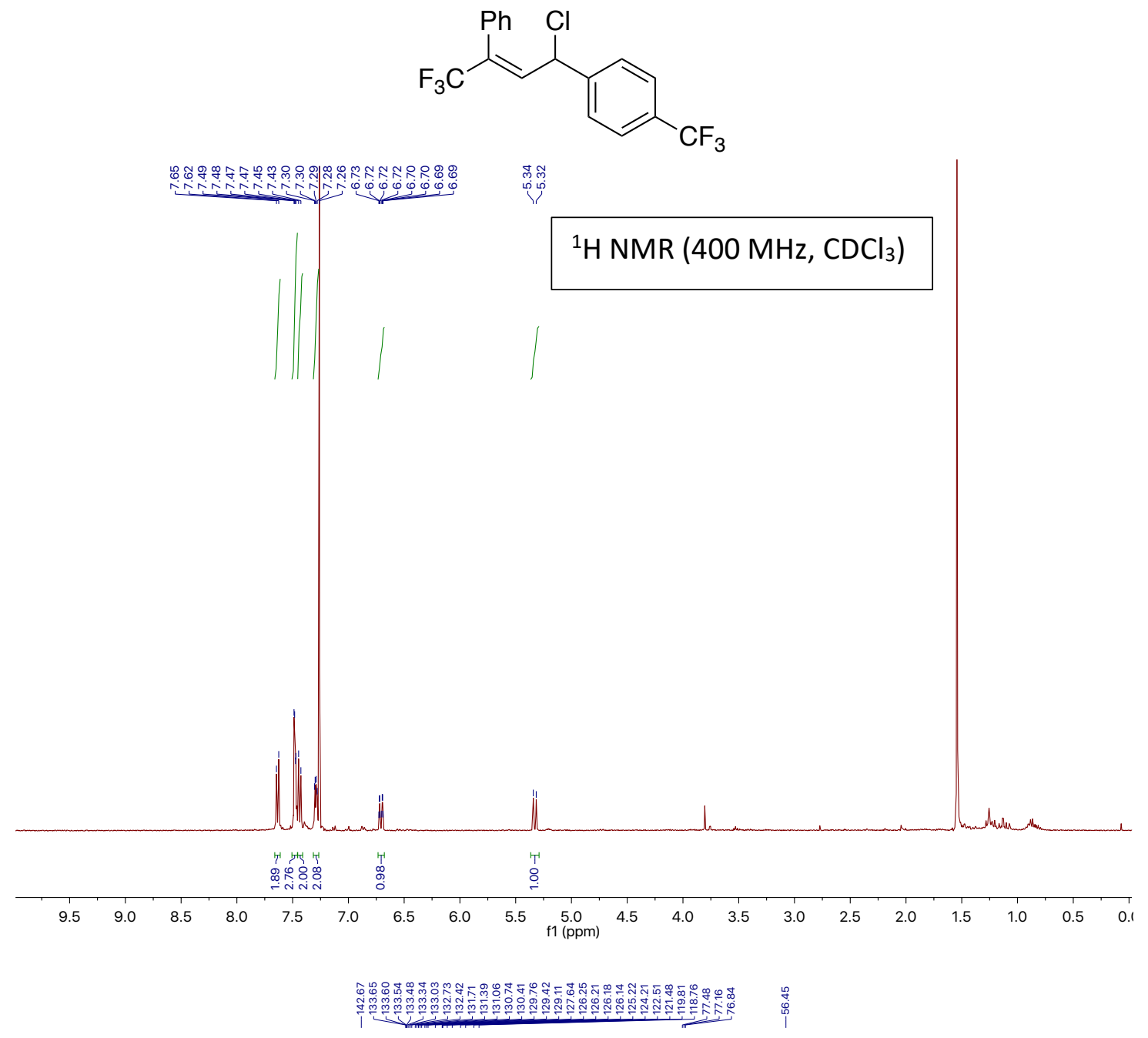

${ }^{13} \mathrm{C} \mathrm{NMR}\left(100 \mathrm{MHz}, \mathrm{CDCl}_{3}\right)$

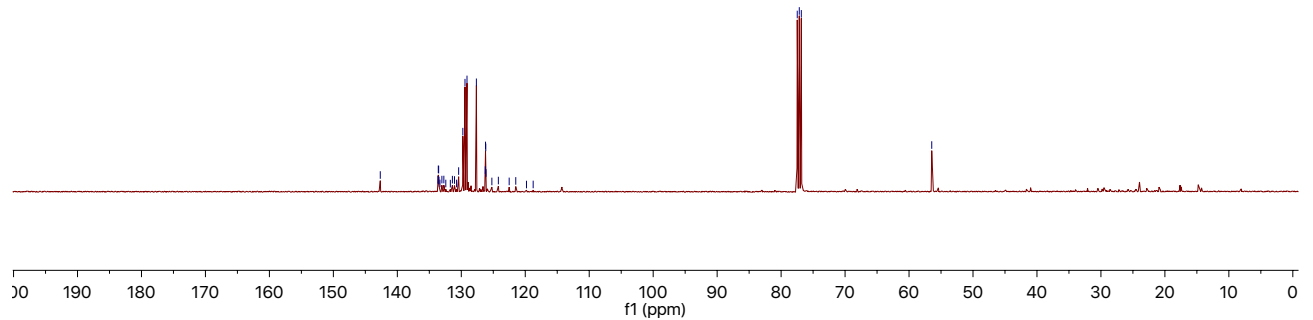


(E)-4-(1-chloro-4,4,4-trifluoro-3-phenylbut-2-en-1-yl)benzonitrile (2d)<smiles>N#Cc1ccc(C(Cl)/C=C(\c2ccccc2)C(F)(F)F)cc1</smiles>

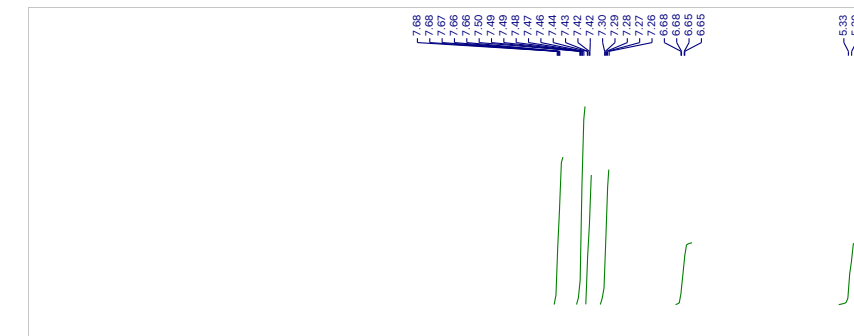

$$
{ }^{1} \mathrm{H} \mathrm{NMR}\left(400 \mathrm{MHz}, \mathrm{CDCl}_{3}\right)
$$
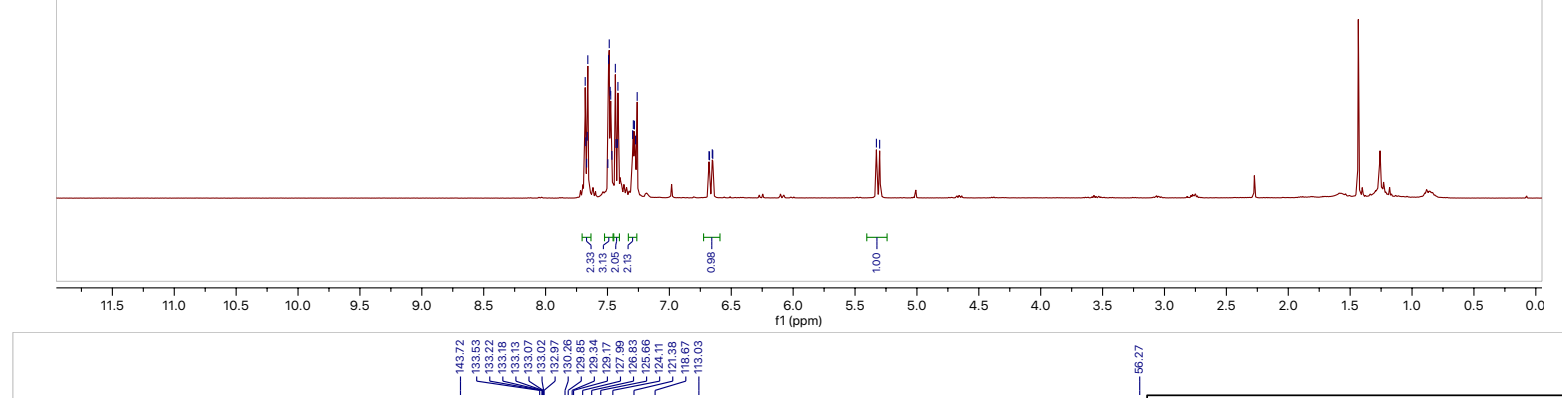

$$
{ }^{13} \mathrm{C} \mathrm{NMR}\left(100 \mathrm{MHz}, \mathrm{CDCl}_{3}\right)
$$

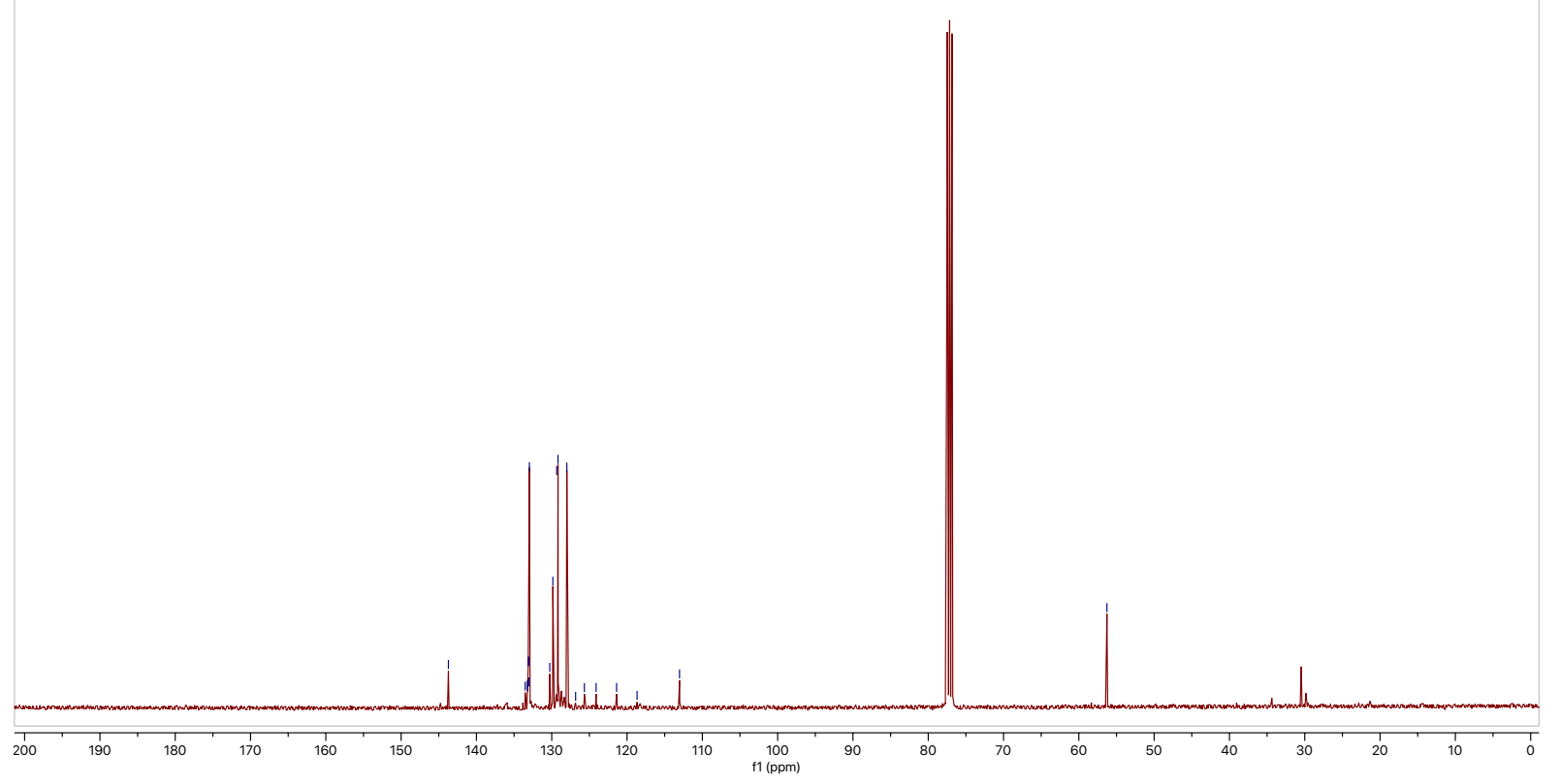

S6 
(E)-1-(1-chloro-4,4,4-trifluoro-3-phenylbut-2-en-1-yl)-4-(methylsulfonyl)benzene (2e)

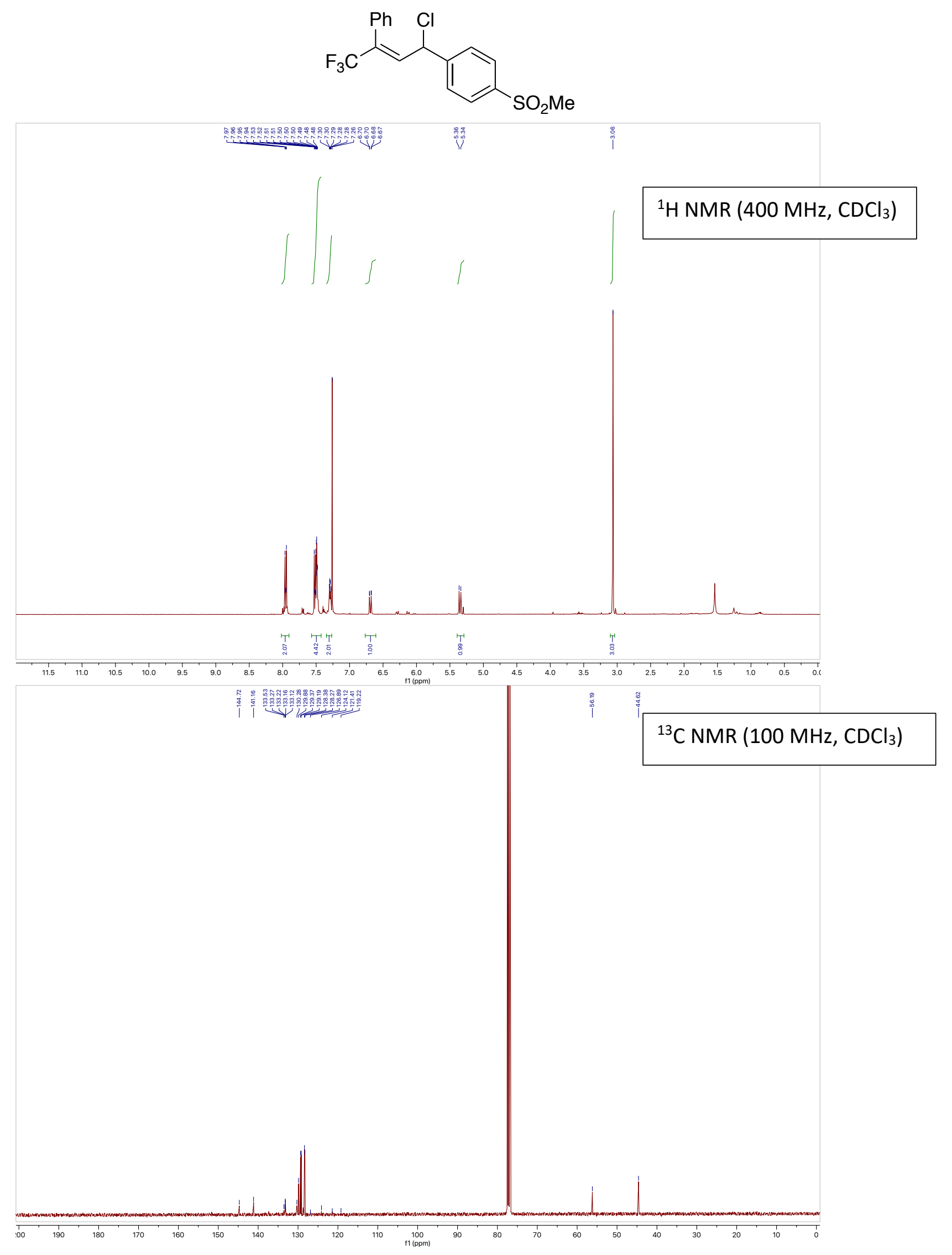


(E)-1-(1-chloro-4,4,4-trifluoro-3-phenylbut-2-en-1-yl)-4-methylbenzene (2f)<smiles>Cc1ccc(C(Cl)/C=C(\c2ccccc2)C(F)(F)F)cc1</smiles>

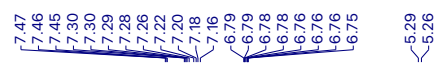

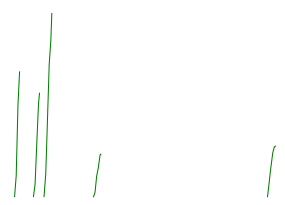

${ }^{1} \mathrm{H}$ NMR $\left(400 \mathrm{MHz}, \mathrm{CDCl}_{3}\right)$

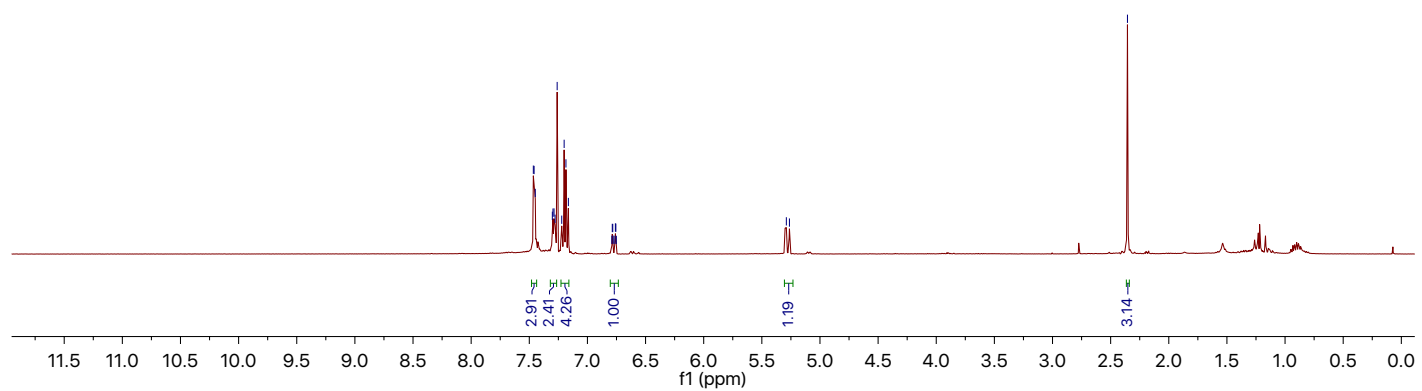

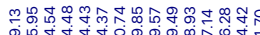

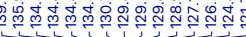

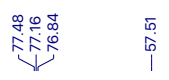

$\stackrel{\substack{i \\ i}}{i}$

${ }^{13} \mathrm{C}$ NMR $\left(100 \mathrm{MHz}, \mathrm{CDCl}_{3}\right)$

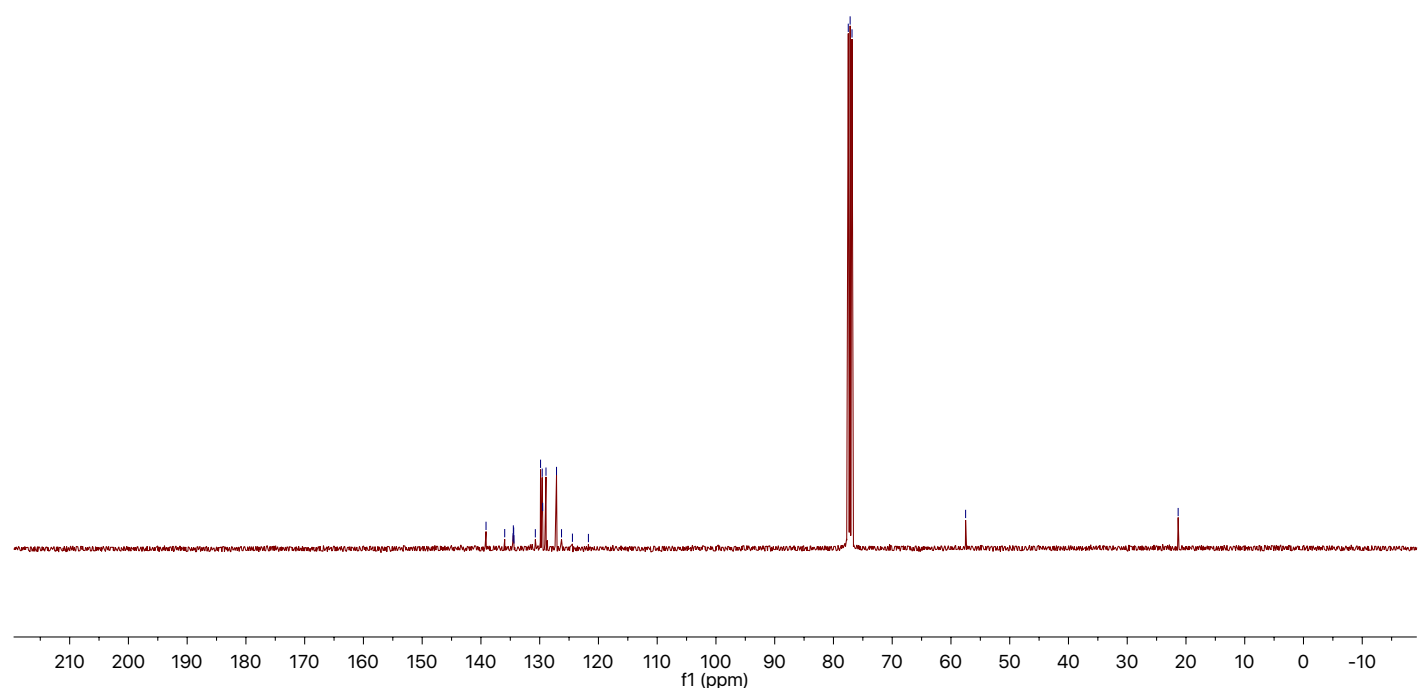


(E)-2-(1-chloro-4,4,4-trifluoro-3-phenylbut-2-en-1-yl)naphthalene (2g)<smiles>FC(F)(F)C(=CC(Cl)c1ccc2ccccc2c1)c1ccccc1</smiles>

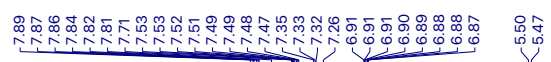

${ }^{1} \mathrm{H} \mathrm{NMR}\left(400 \mathrm{MHz}, \mathrm{CDCl}_{3}\right)$

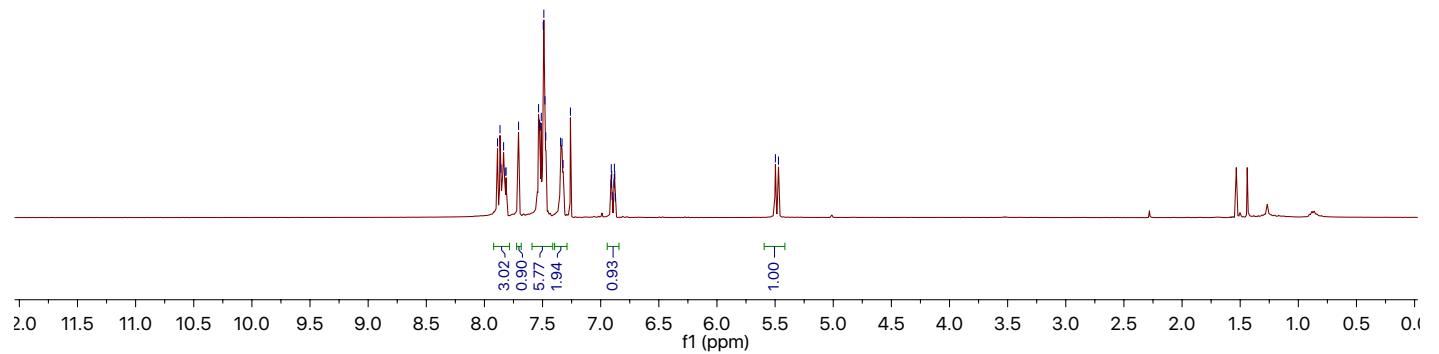

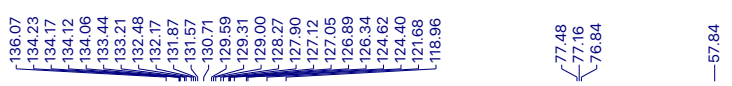

${ }^{13} \mathrm{C}$ NMR $\left(100 \mathrm{MHz}, \mathrm{CDCl}_{3}\right)$

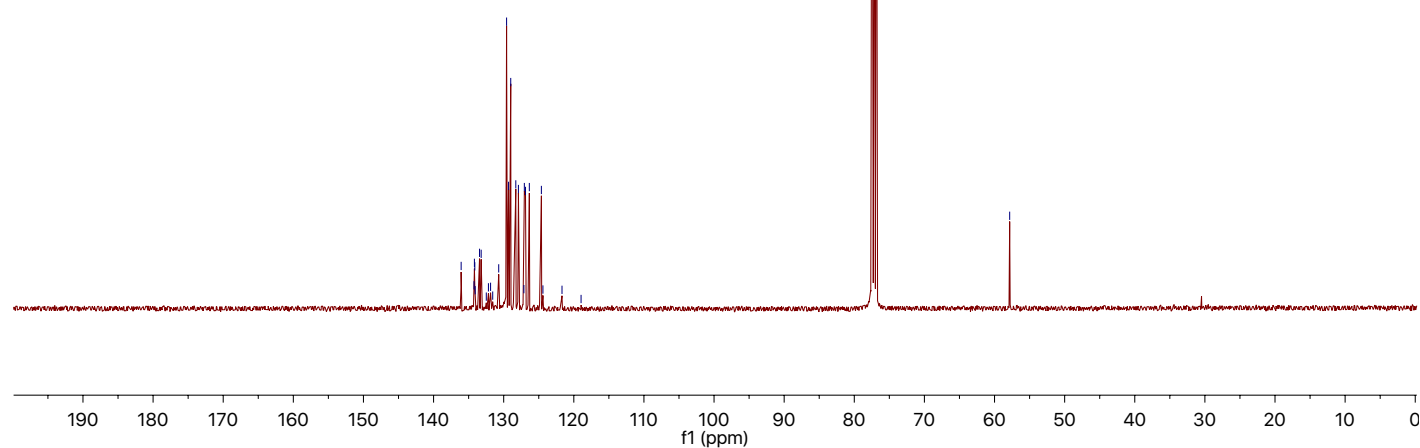


(E)-(4-chloro-1,1,1-trifluoropent-2-en-2-yl)benzene (2h)<smiles>CC(Cl)/C=C(\c1ccccc1)C(F)(F)Cl</smiles>

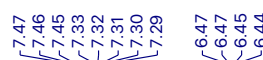

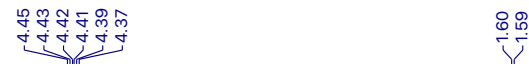

${ }^{1} \mathrm{H}$ NMR (400 MHz, $\mathrm{CDCl}_{3}$ )

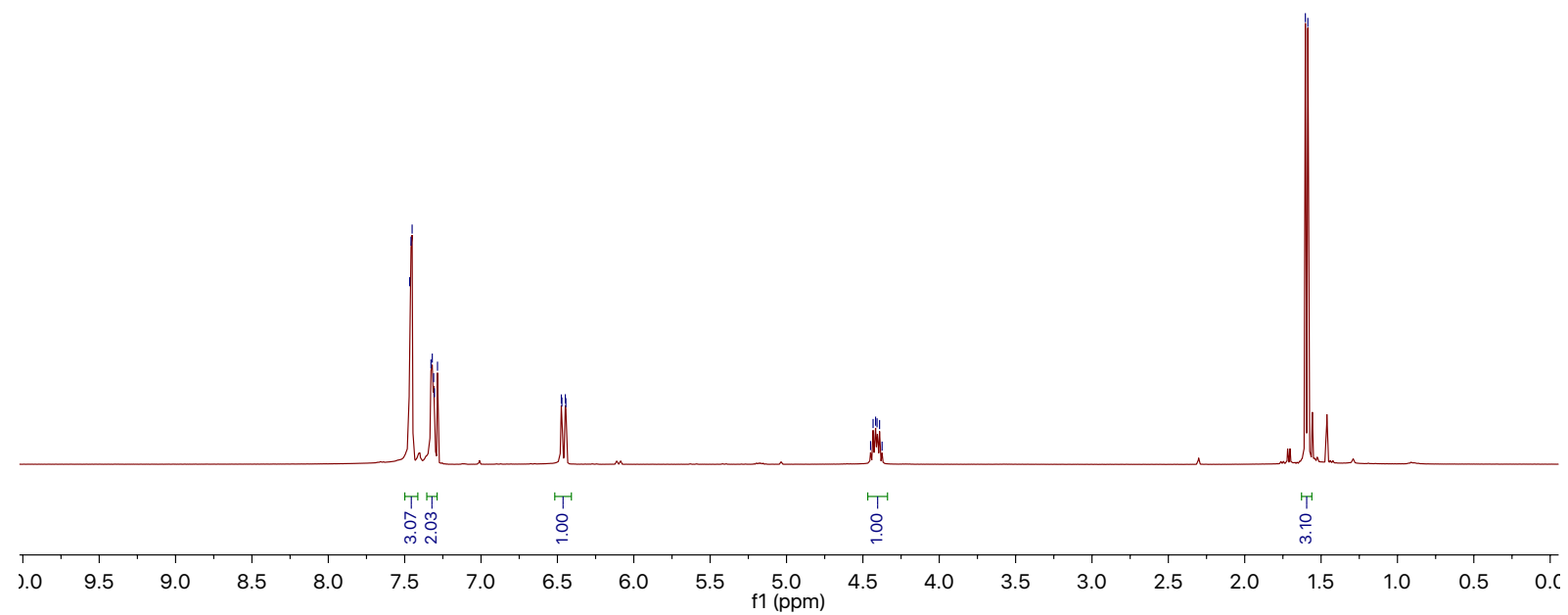


${ }^{13} \mathrm{C}$ NMR $\left(100 \mathrm{MHz}, \mathrm{CDCl}_{3}\right)$
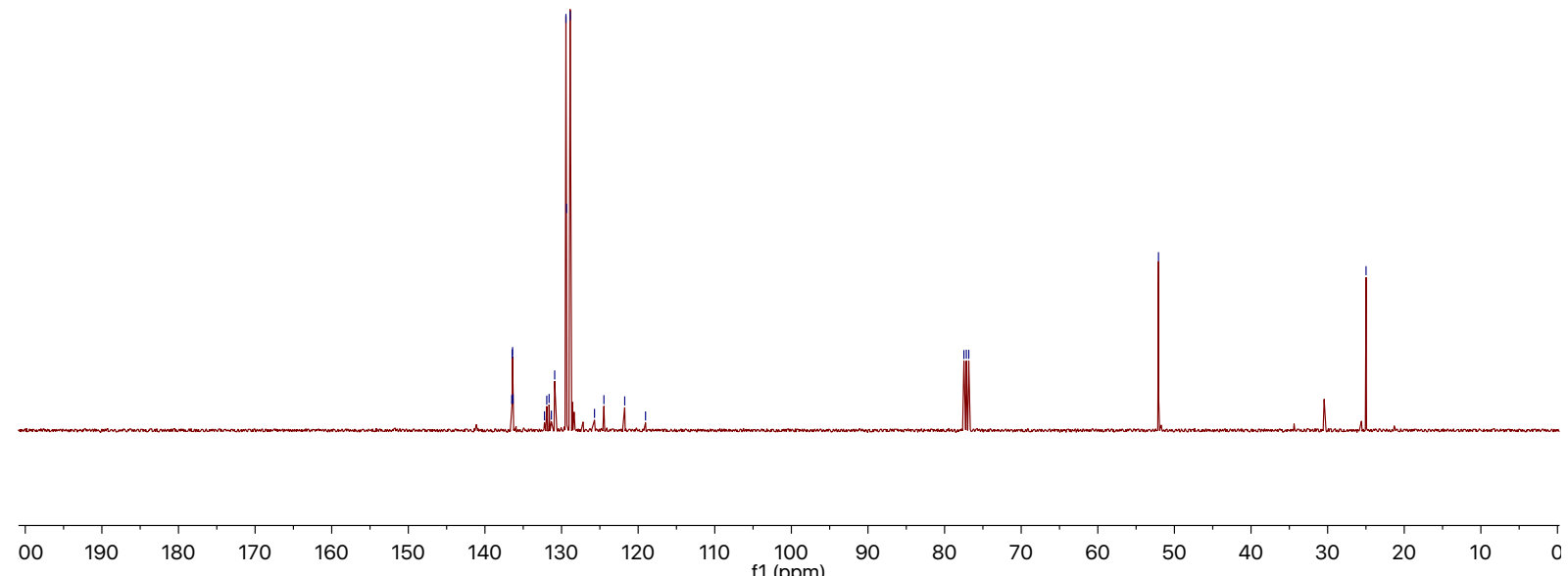

(E)-(4-chloro-1,1,1-trifluorobut-2-en-2-yl)benzene (2i)<smiles>FC(F)(F)/C(=C/CCl)c1ccccc1</smiles>

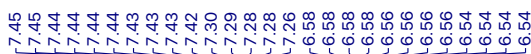

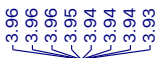

${ }^{1} \mathrm{H}$ NMR $\left(400 \mathrm{MHz}, \mathrm{CDCl}_{3}\right)$

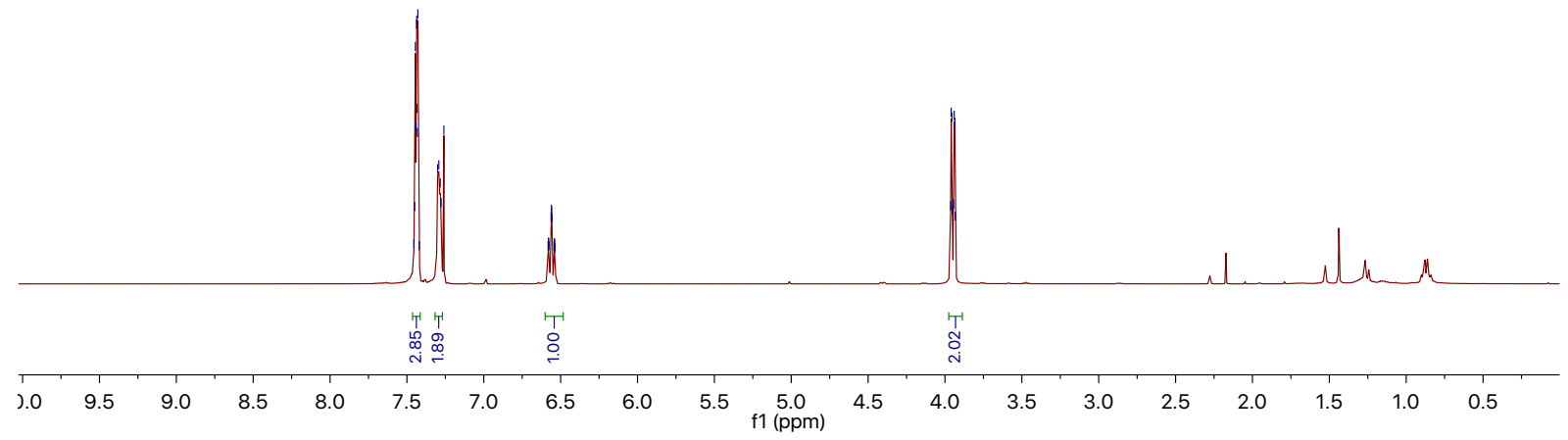




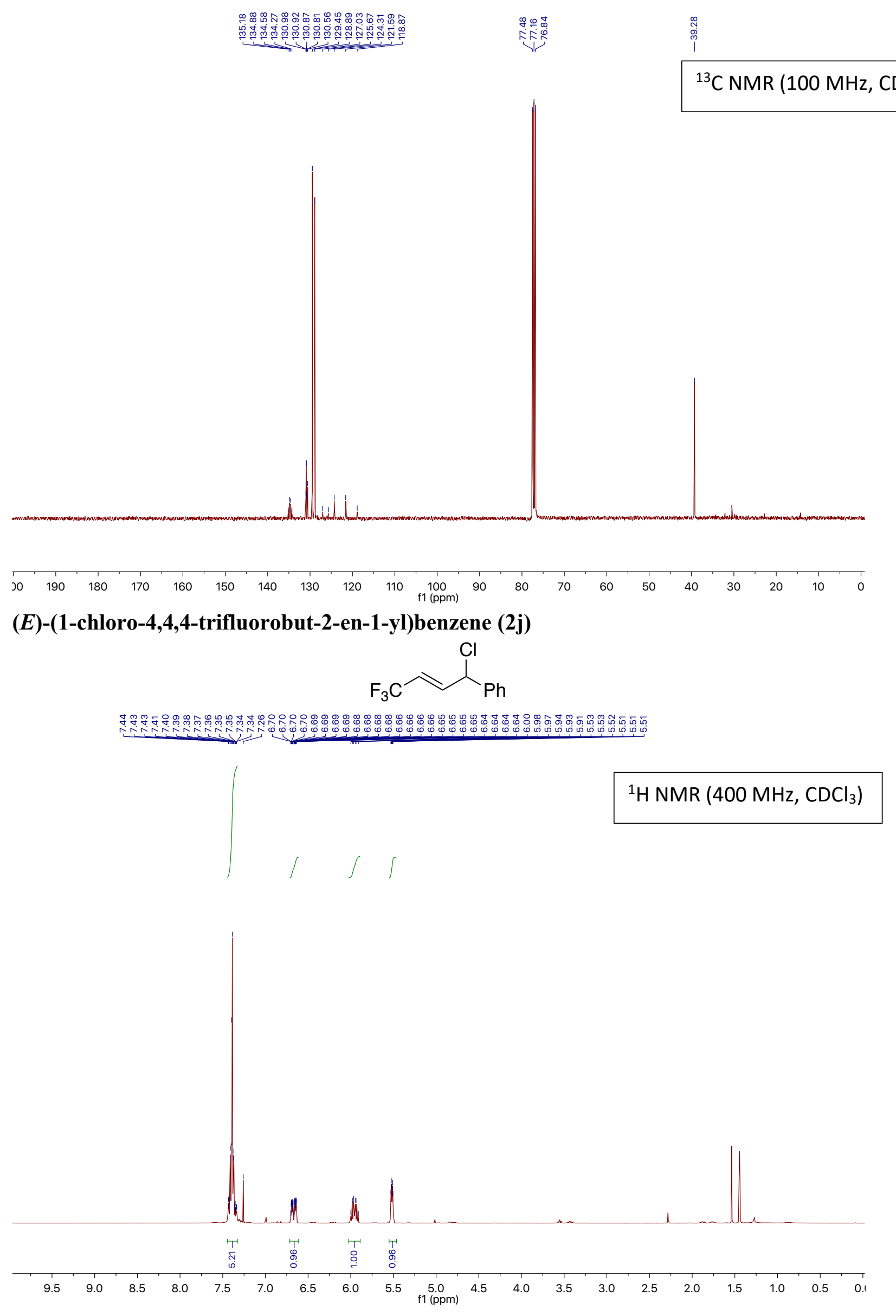


$\left.{ }^{13} \mathrm{C} \mathrm{NMR} \mathrm{(100} \mathrm{MHz,} \mathrm{CDCl} 3\right)$

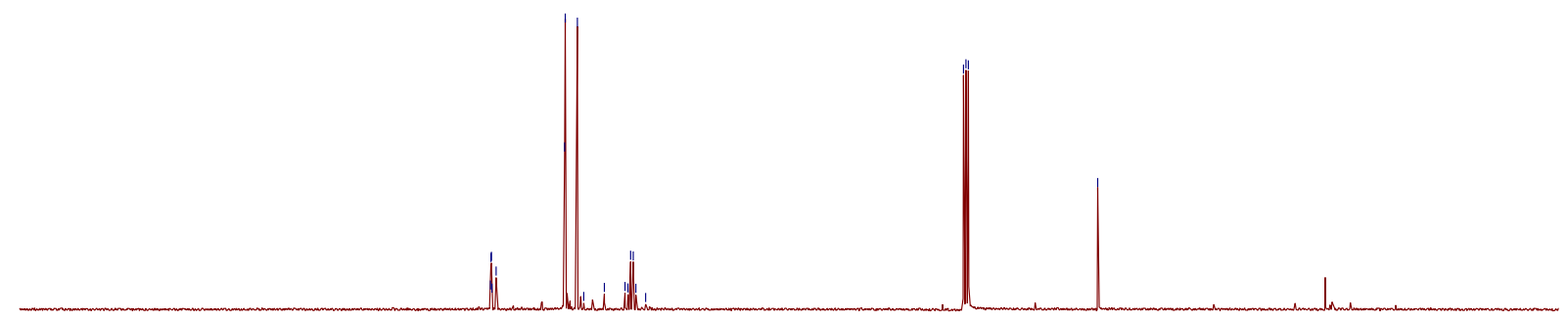

(E)-(1-chloro-4,4,4-trifluoro-3-methylbut-2-en-1-yl)benzene (2k)<smiles>CC(=CC(Cl)c1ccccc1)C(F)(F)F</smiles> 


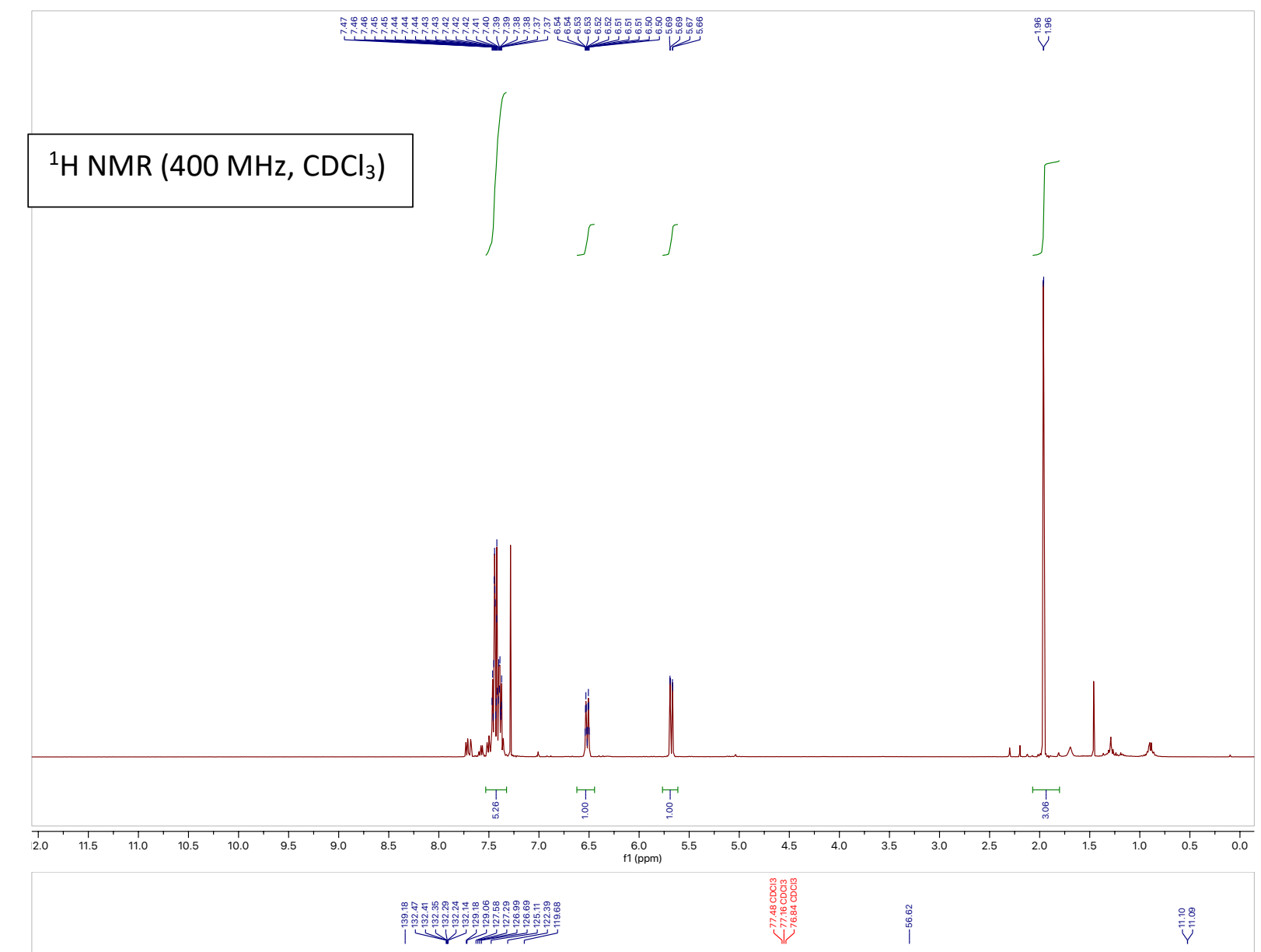

${ }^{13} \mathrm{C} \mathrm{NMR}\left(100 \mathrm{MHz}, \mathrm{CDCl}_{3}\right)$

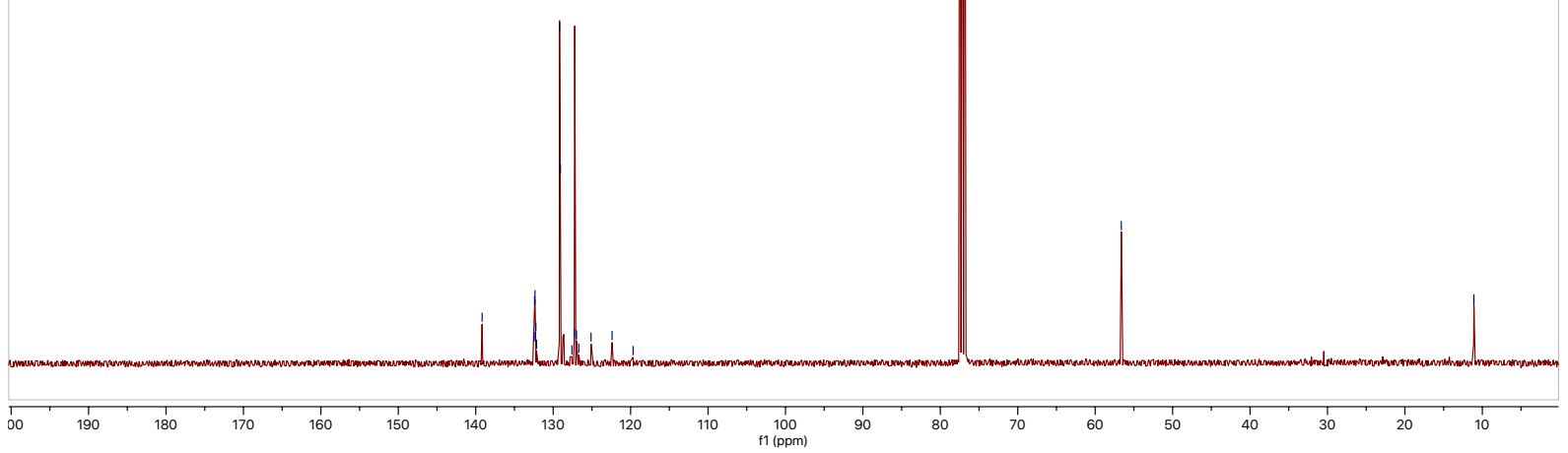

(E)-1-chloro-4-(4-chloro-1,1,1-trifluoro-4-phenylbut-2-en-2-yl)benzene (2l) 


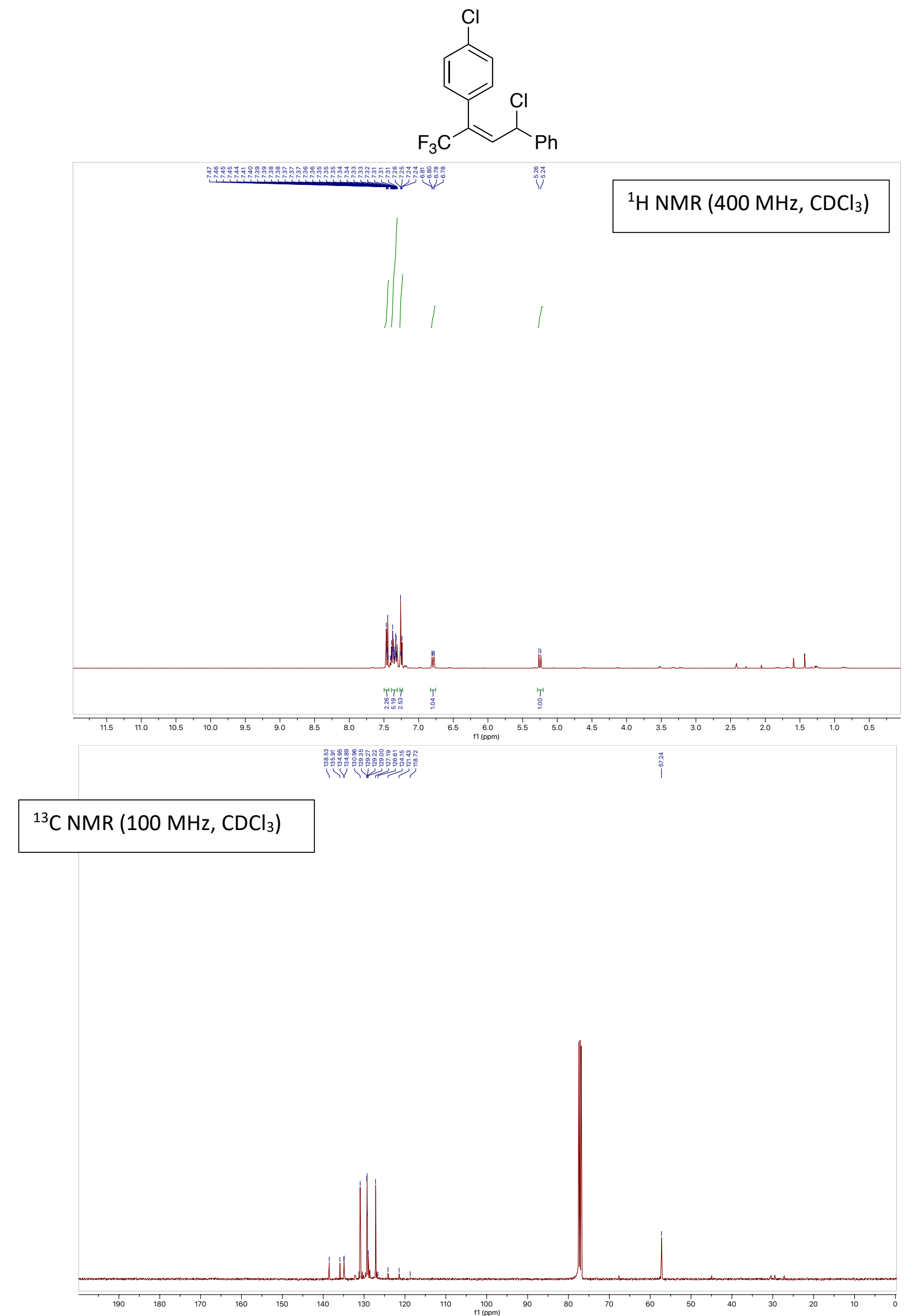

(E)-1-(4-Chloro-1,1,1-trifluoro-4-phenylbut-2-en-2-yl)-4-(trifluoromethyl)benzene (2m) 


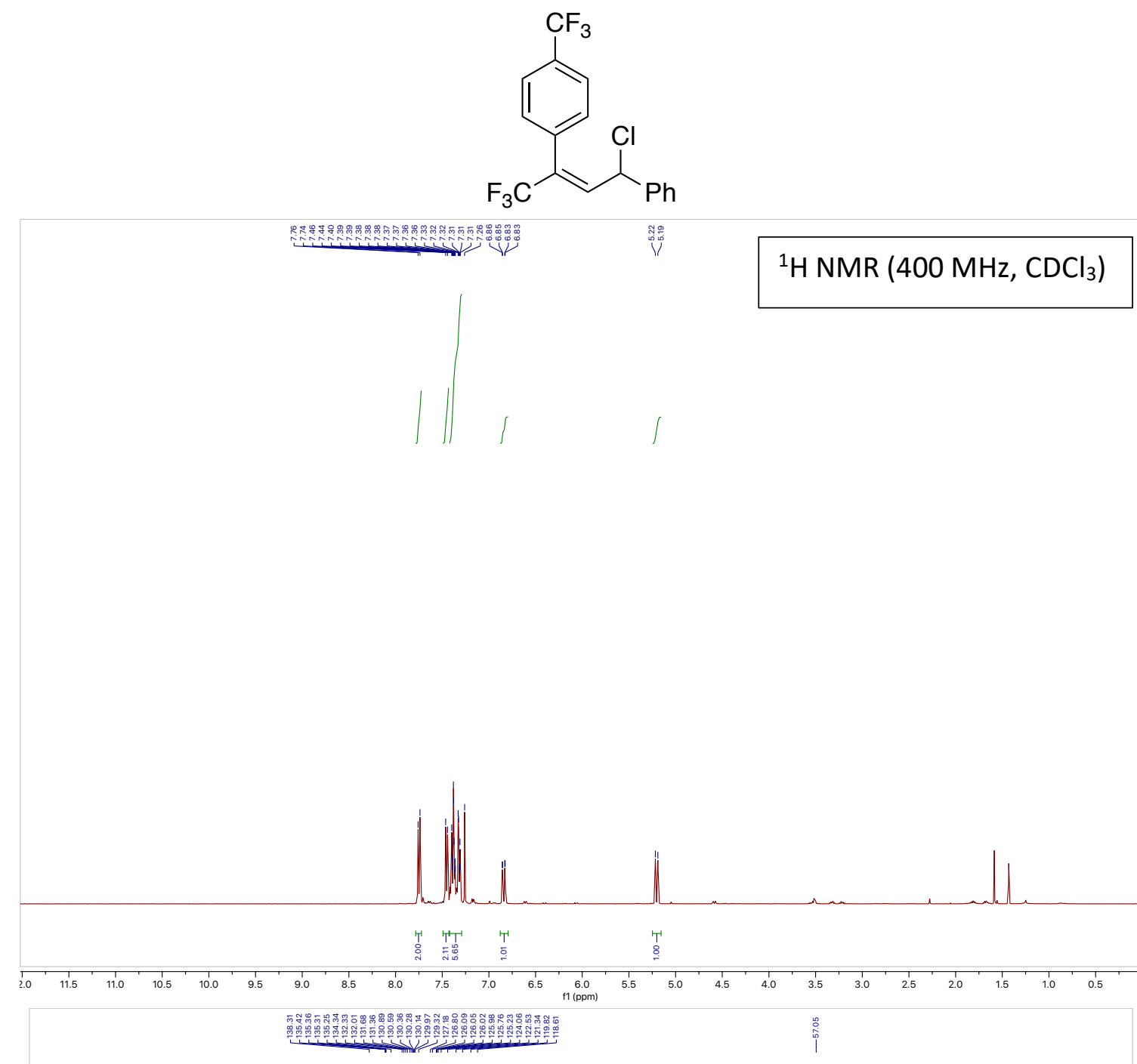

${ }^{13} \mathrm{C} \mathrm{NMR}\left(100 \mathrm{MHz}, \mathrm{CDCl}_{3}\right)$

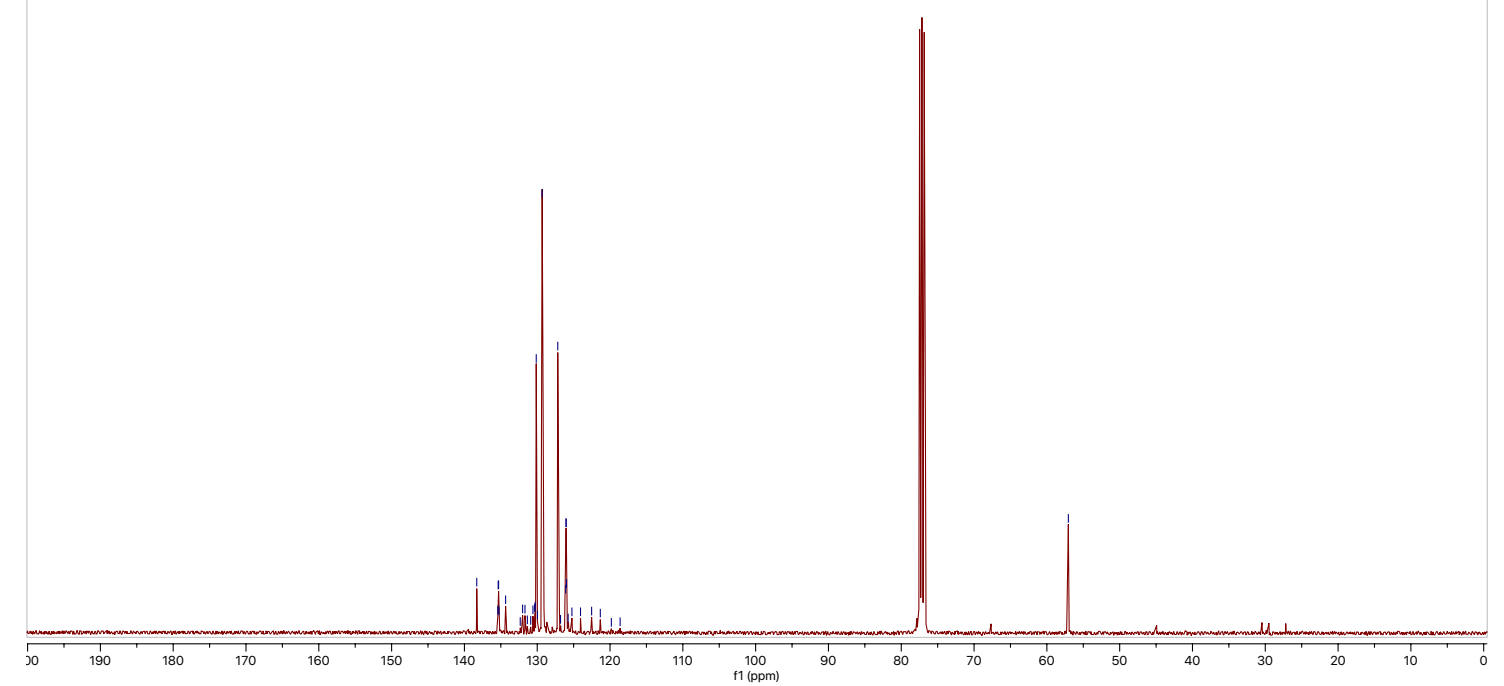

(E)-(3-chloro-1-(phenylsulfonyl)prop-1-ene-1,3-diyl)dibenzene $((E)-2 n)$ 

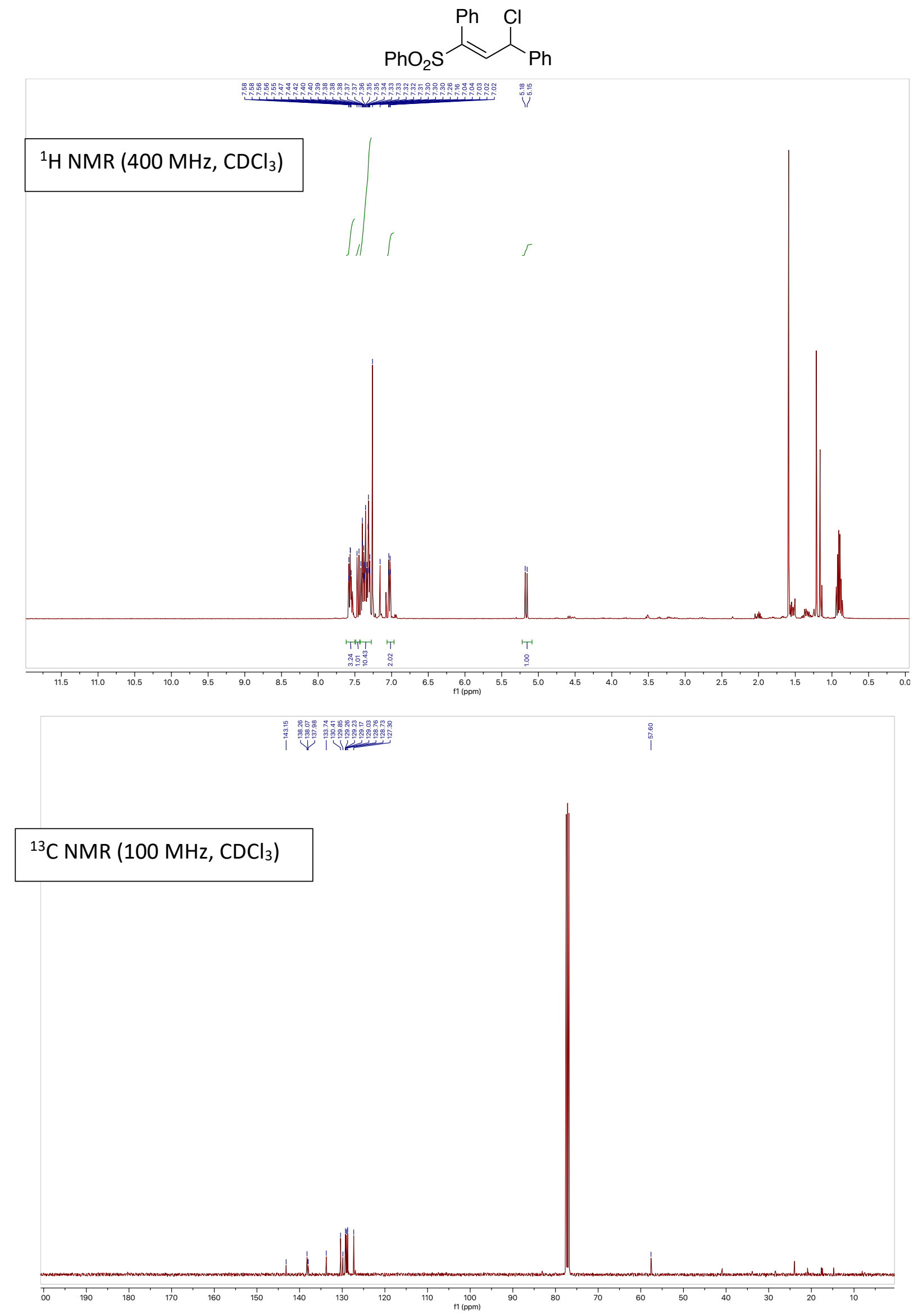

(Z)-(3-chloro-1-(phenylsulfonyl)prop-1-ene-1,3-diyl)dibenzene ((Z)-2n) 


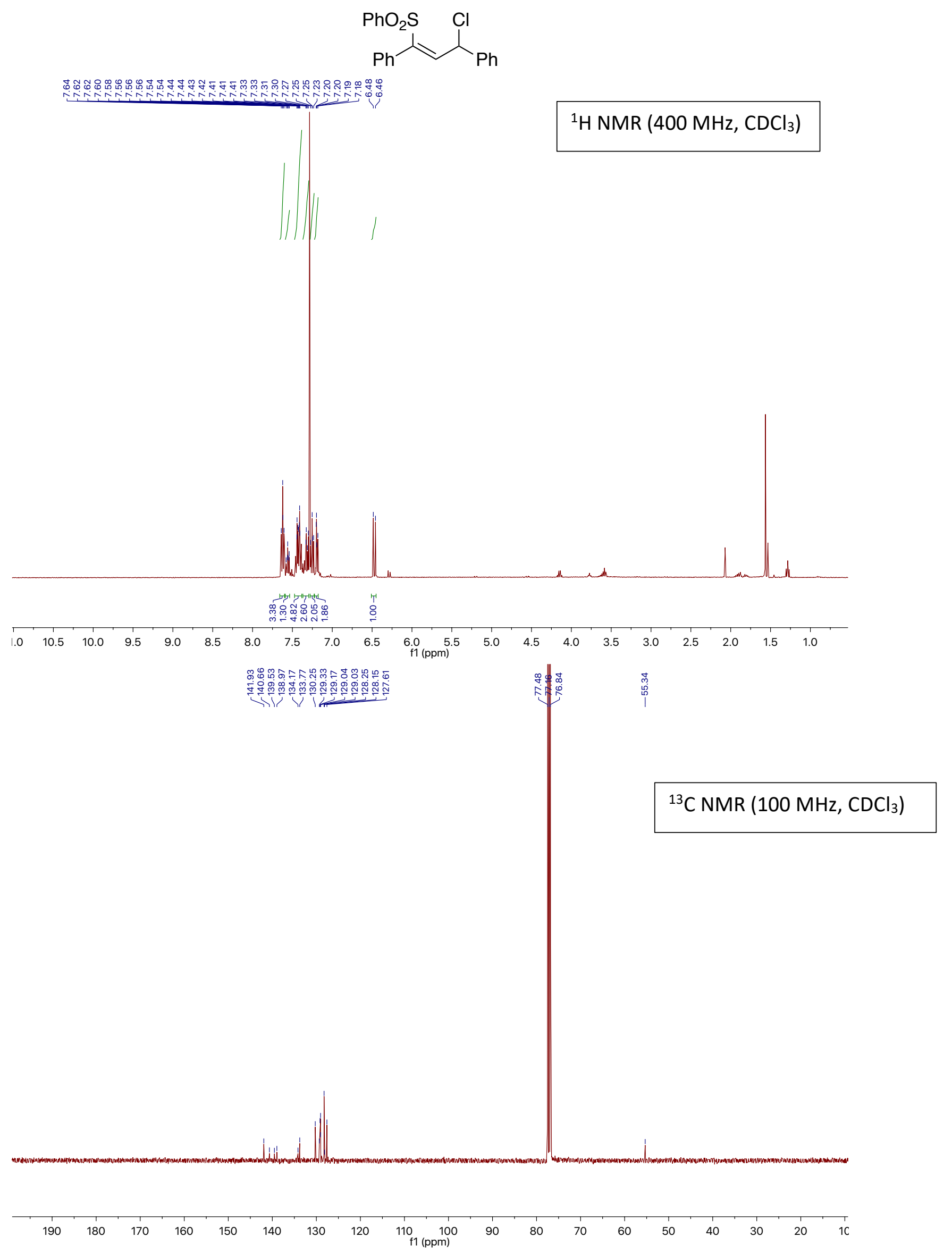

(Z)-(1-chloro-2,4,4,4-tetrafluorobut-2-ene-1,3-diyl)dibenzene (2o) 


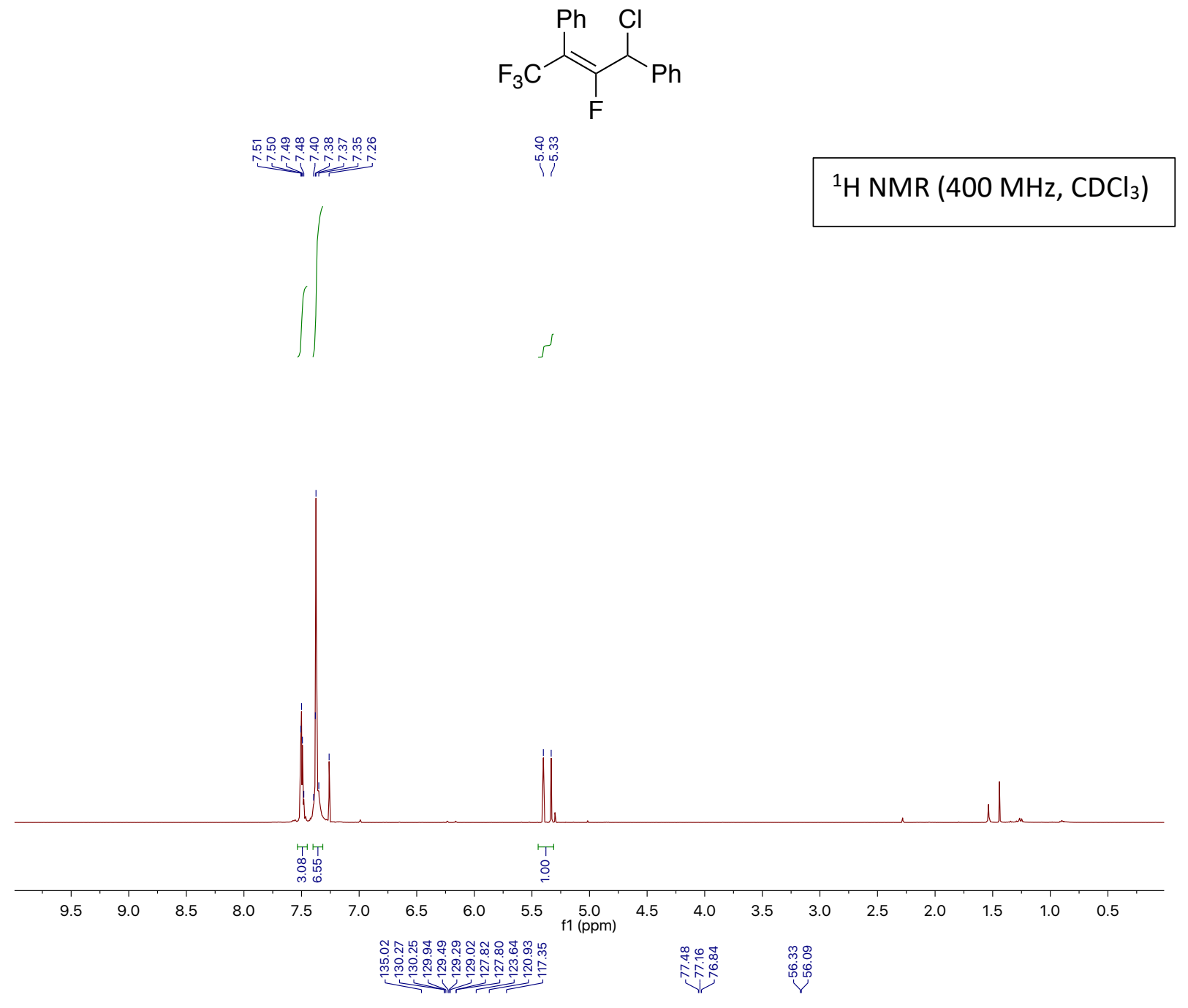

${ }^{13} \mathrm{CNMR}\left(100 \mathrm{MHz}, \mathrm{CDCl}_{3}\right)$

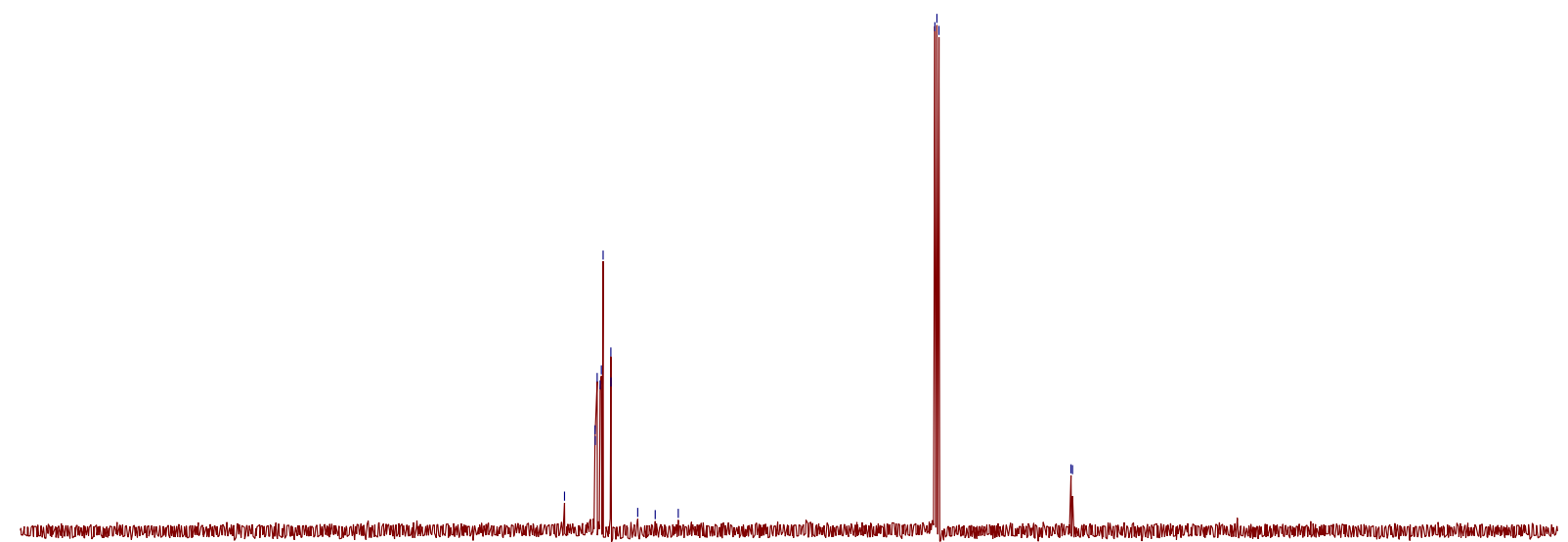

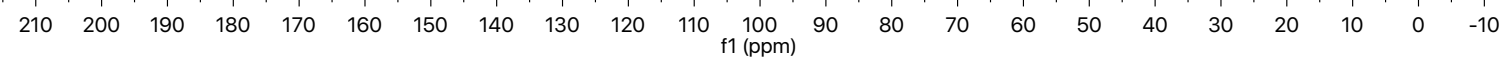

(E)-(1-bromo-4,4,4-trifluorobut-2-ene-1,3-diyl)dibenzene (3a) 


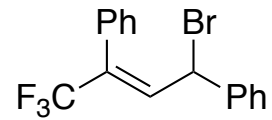

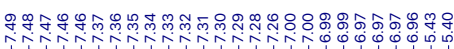

${ }^{1} \mathrm{H}$ NMR $\left(400 \mathrm{MHz}, \mathrm{CDCl}_{3}\right)$

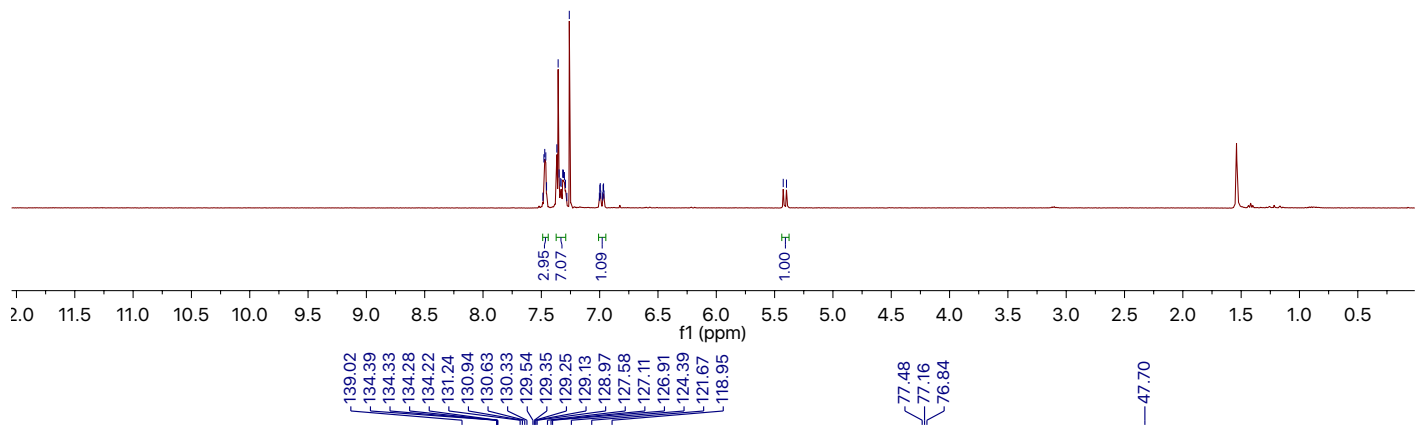

${ }^{13} \mathrm{C}$ NMR $\left(100 \mathrm{MHz}, \mathrm{CDCl}_{3}\right)$

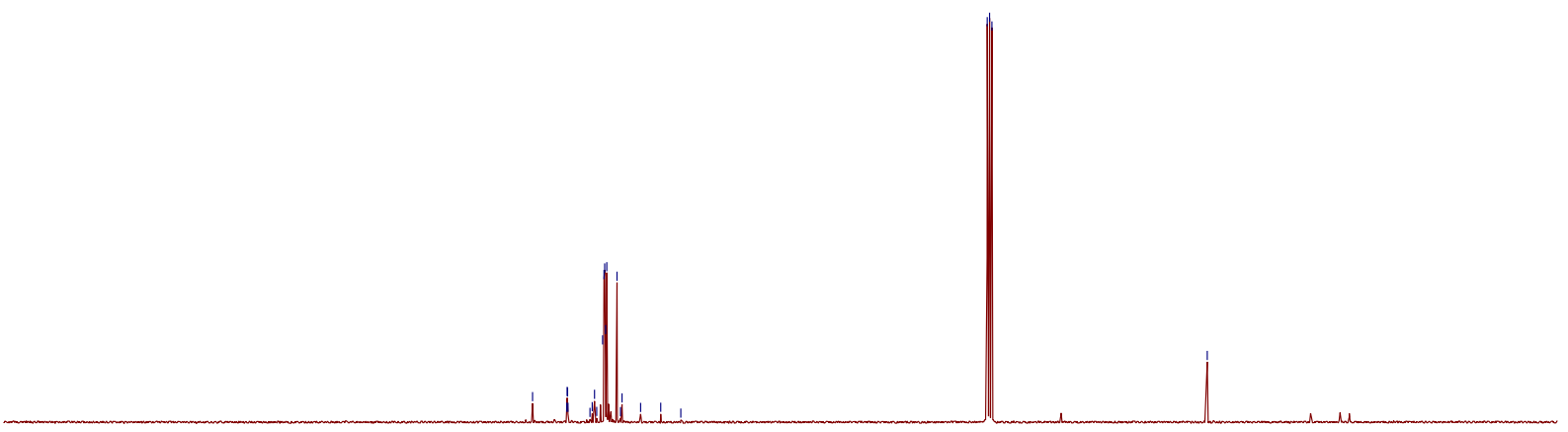

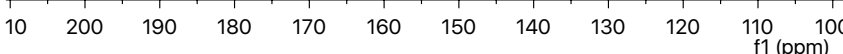

(E)-1-(1-bromo-4,4,4-trifluoro-3-phenylbut-2-en-1-yl)-4-(trifluoromethyl)benzene (3b) 


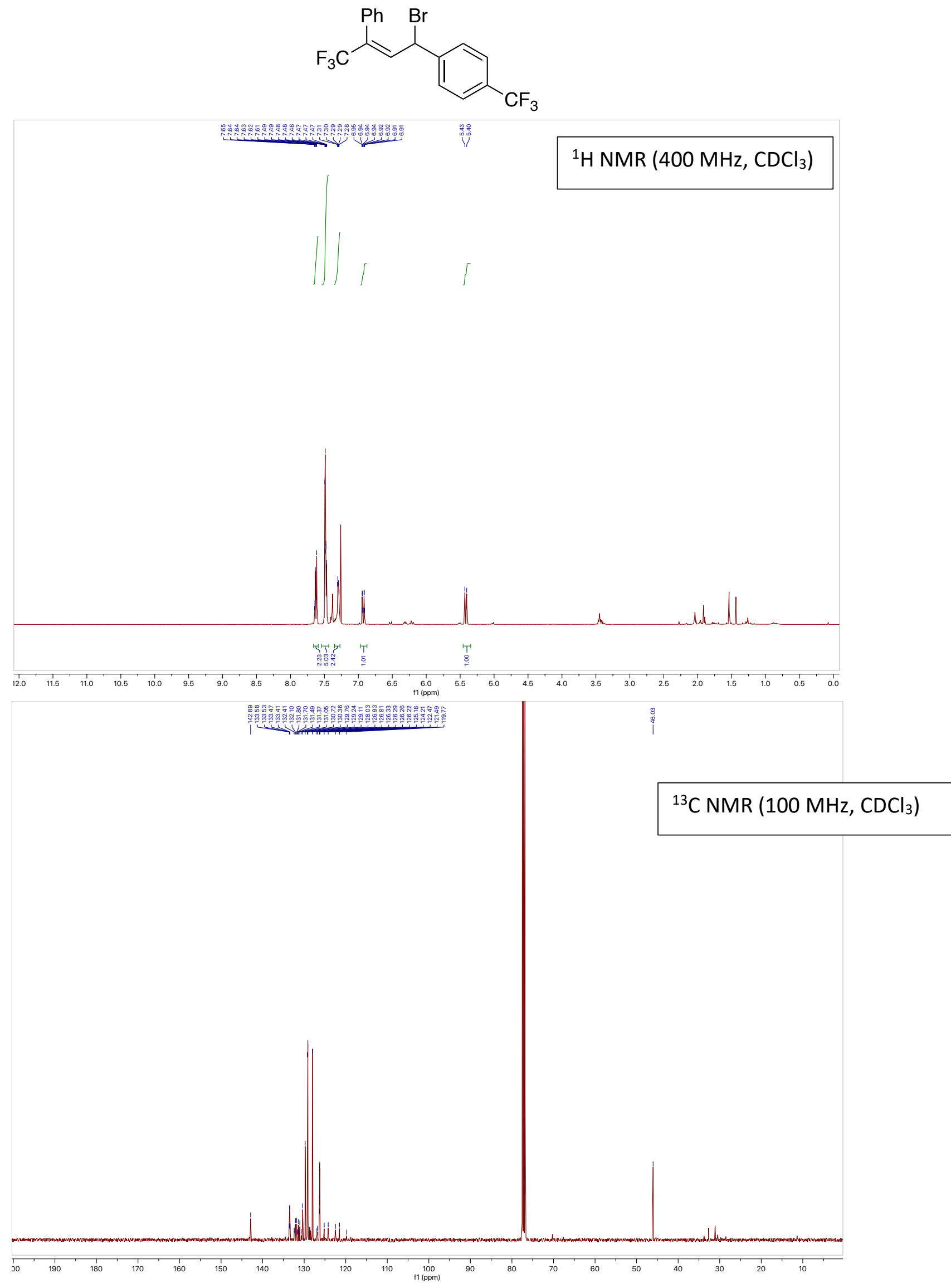

(E)-(3-bromo-1-(phenylsulfonyl)prop-1-ene-1,3-diyl)dibenzene (3c) 


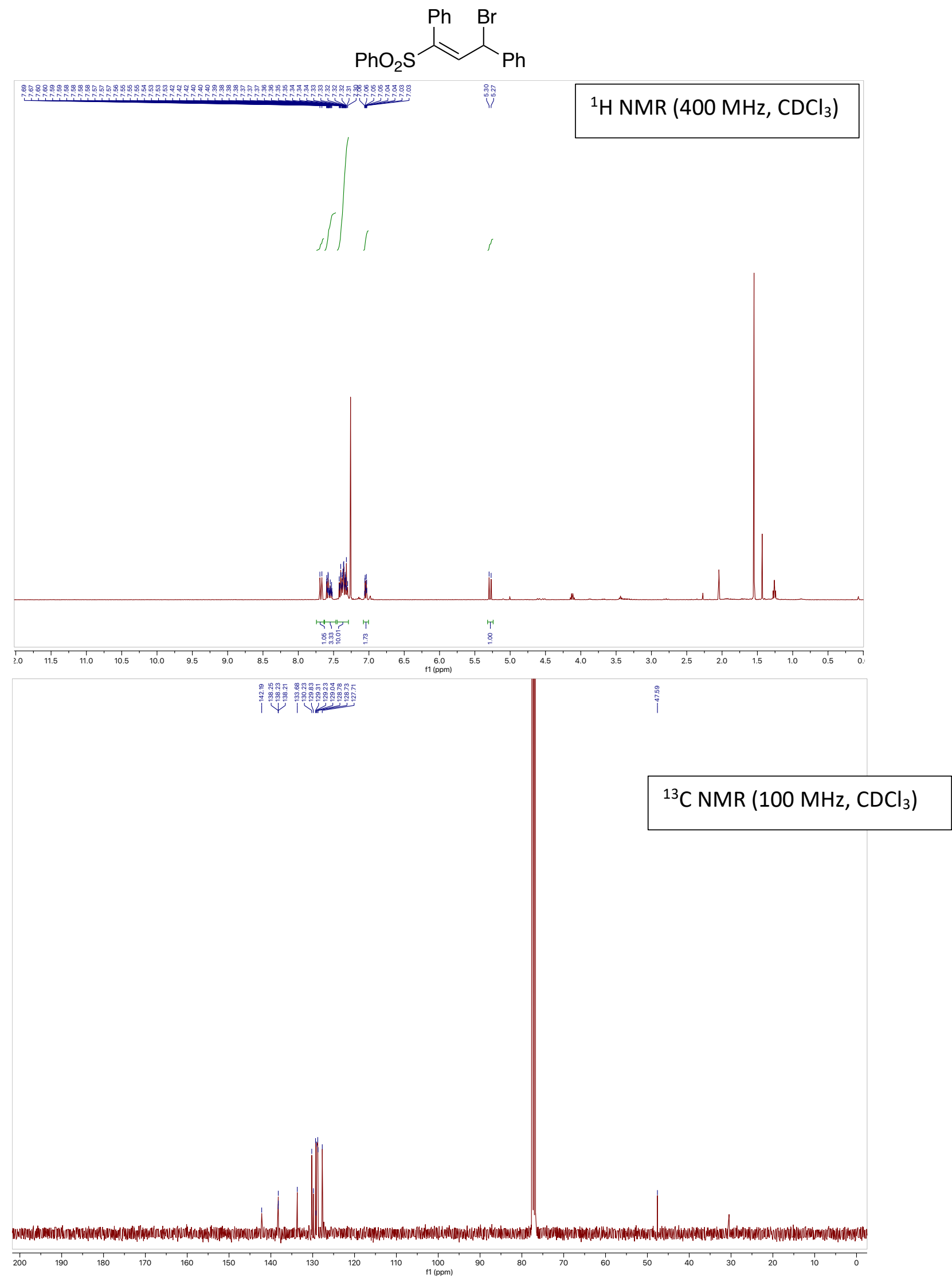

(E)-(1-fluoro-4,4,4-trifluorobut-2-ene-1,3-diyl)dibenzene (4a) 
<smiles>FC(/C=C(/c1ccccc1)C(F)(F)F)c1ccccc1</smiles>

$\underbrace{-15}$

${ }^{1} \mathrm{H}$ NMR $\left(400 \mathrm{MHz}, \mathrm{CDCl}_{3}\right)$
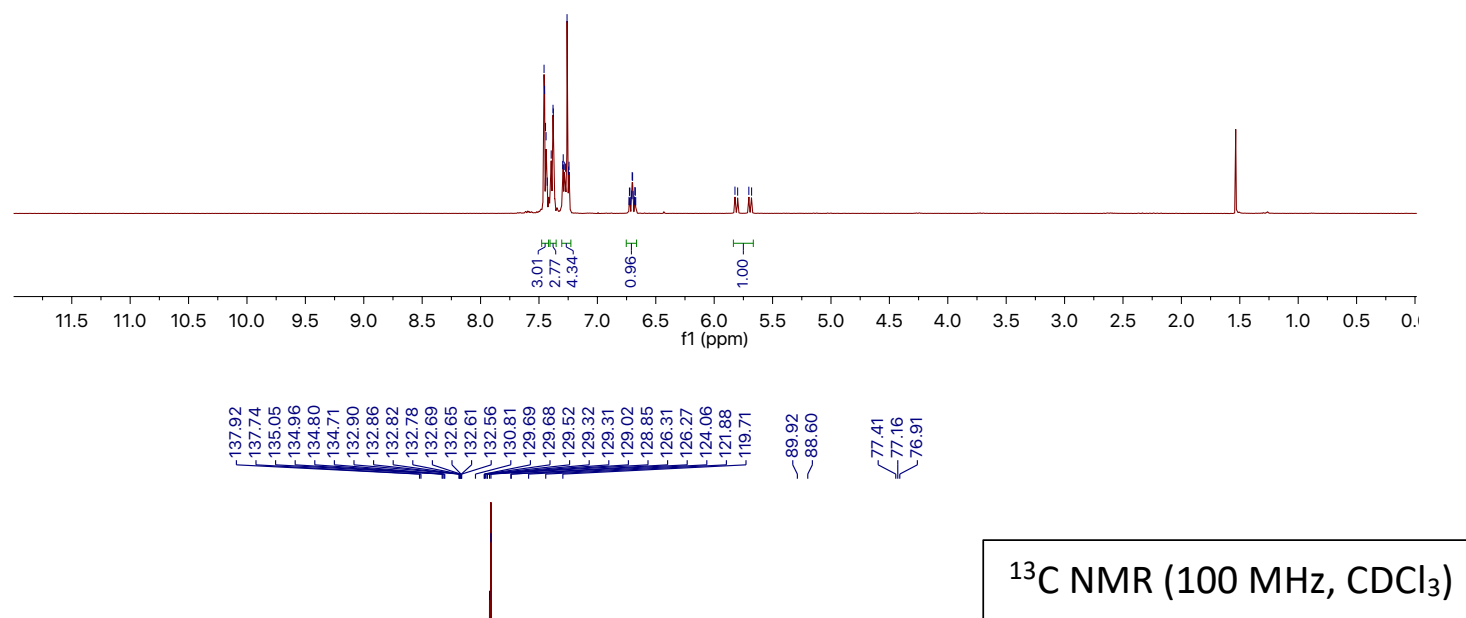

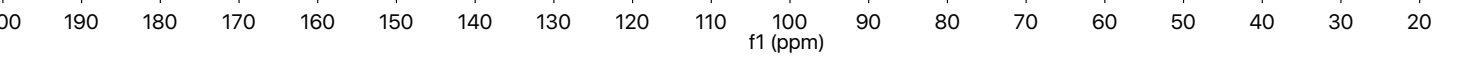

(E)-1-(1,4,4,4-tetrafluoro-3-phenylbut-2-en-1-yl)-4-(trifluoromethyl)benzene (4b) 

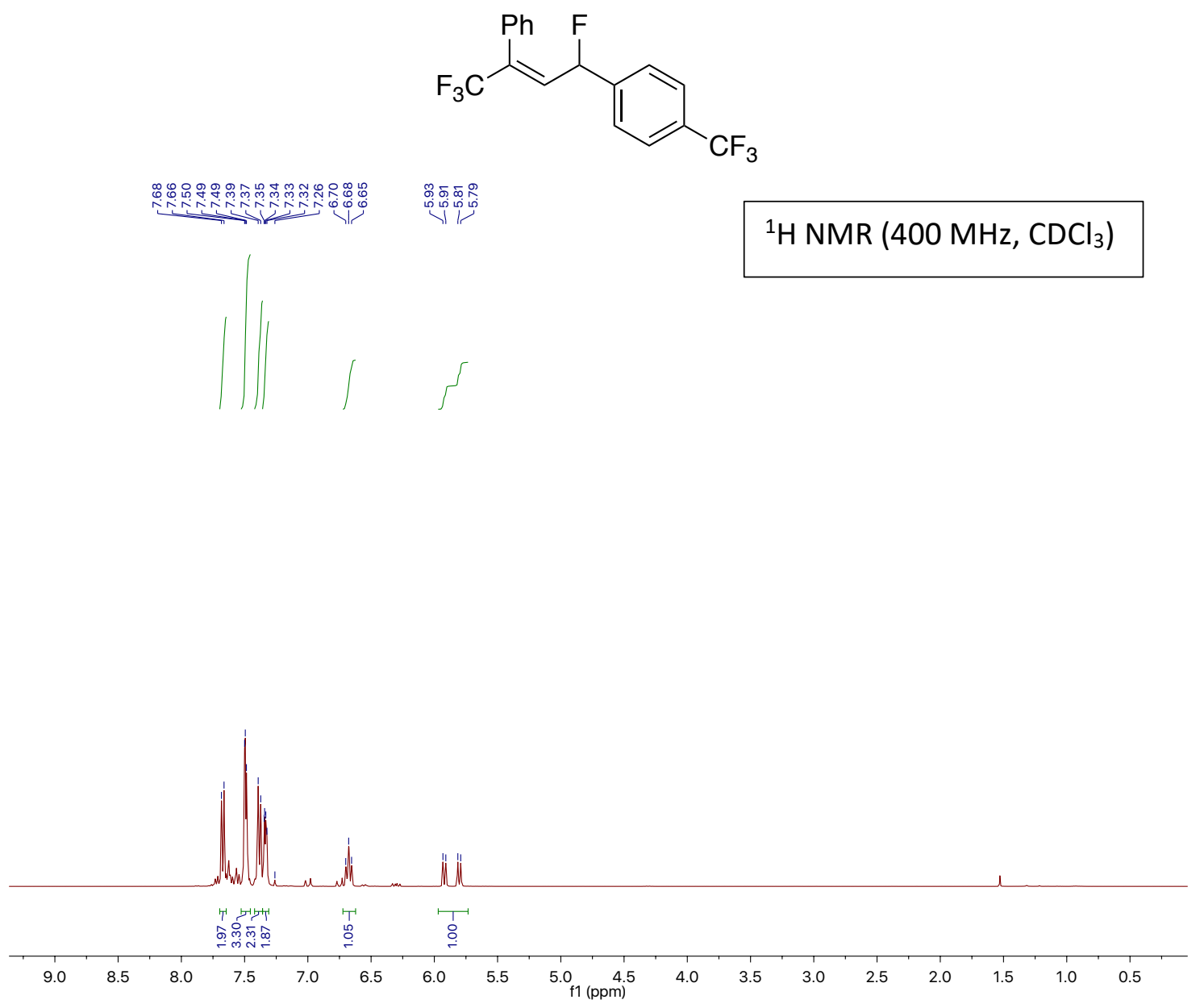

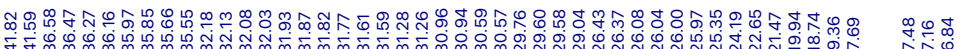

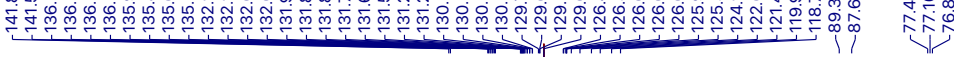

${ }^{13} \mathrm{C} \mathrm{NMR}\left(100 \mathrm{MHz}, \mathrm{CDCl}_{3}\right)$

$\begin{array}{lllllllllllllllllllll}210 & 200 & 190 & 180 & 170 & 160 & 150 & 140 & 130 & 120 & \begin{array}{c}110 \\ \mathrm{f} 1(\mathrm{ppm})\end{array} & \mathbf{1 0 0} & \mathbf{1 0} & \mathbf{1} & 70 & 60 & 50 & 40 & 30 & 20 & 10\end{array}$

(E)-2-(1,4,4,4-tetrafluoro-3-phenylbut-2-en-1-yl)naphthalene (4c) 
<smiles>FC(/C=C(\c1ccccc1)C(F)(F)F)c1ccc2ccccc2c1</smiles>

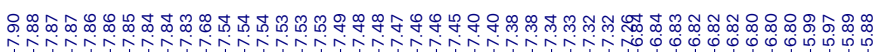
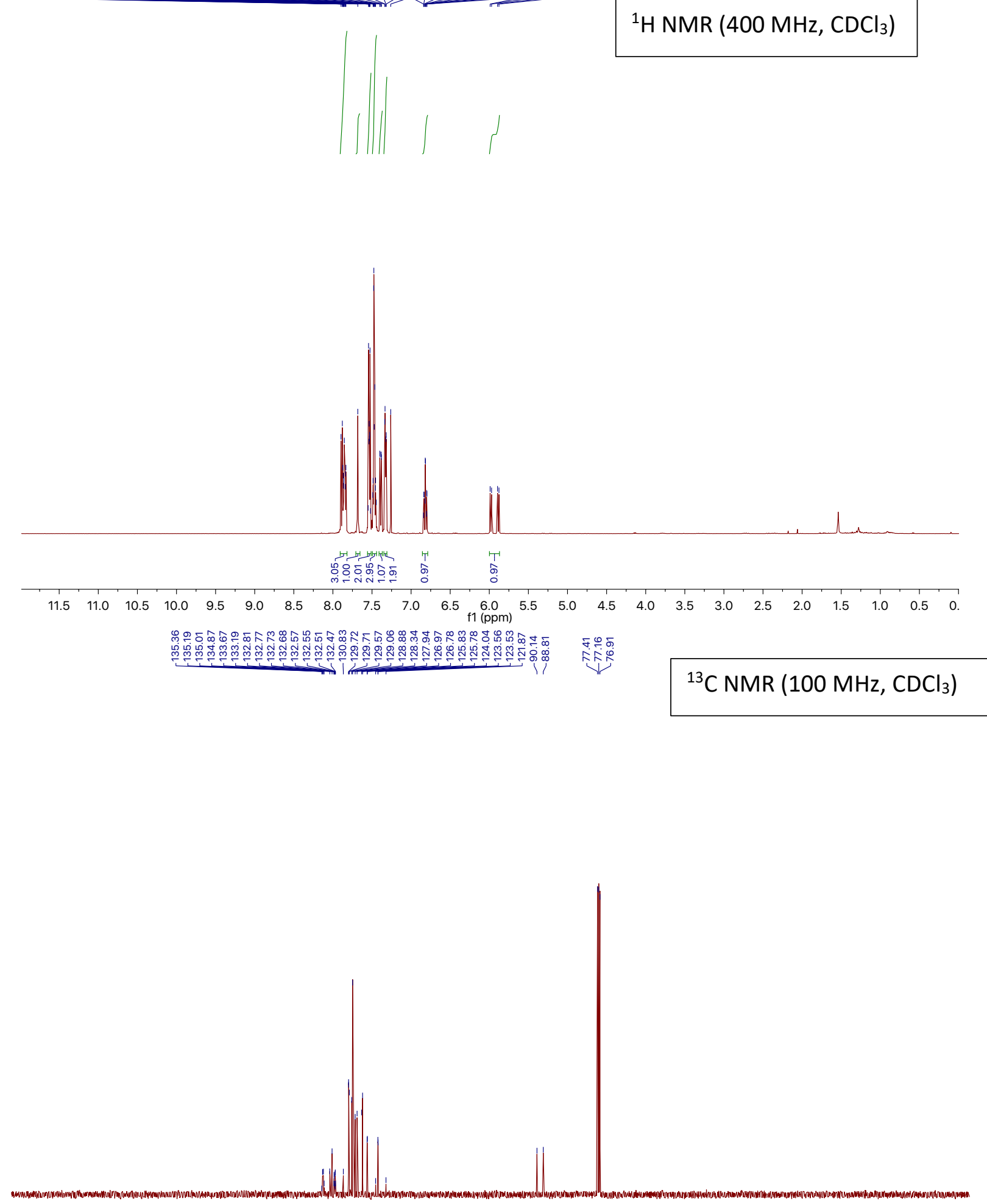

$\begin{array}{lllllllllllllllllllll}00 & 190 & 180 & 170 & 160 & 150 & 140 & 130 & 120 & 110 & 100 & 90 & 80 & 70 & 60 & 50 & 40 & 30 & 20 & 10 & 0\end{array}$ 


\section{$\underline{{ }^{1} \mathrm{H} \text { NMR, }{ }^{13} \mathrm{C} \text { NMR and }{ }^{19} \mathrm{~F} \text { NMR of vinyl halides 5a-5n, 6a-6c and } 7 \mathrm{a}-7 \mathrm{c}}$}

\section{(Z)-(1-chloro-4,4,4-trifluorobut-1-ene-1,3-diyl)dibenzene (5a)}<smiles>FC(F)(F)C(/C=C(\Cl)c1ccccc1)c1ccccc1</smiles>

\begin{tabular}{|c|c|c|}
\hline 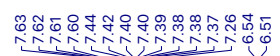 & 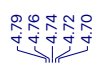 & ${ }^{1} \mathrm{H}$ NMR $\left(400 \mathrm{MHz}, \mathrm{CDCl}_{3}\right)$ \\
\hline
\end{tabular}

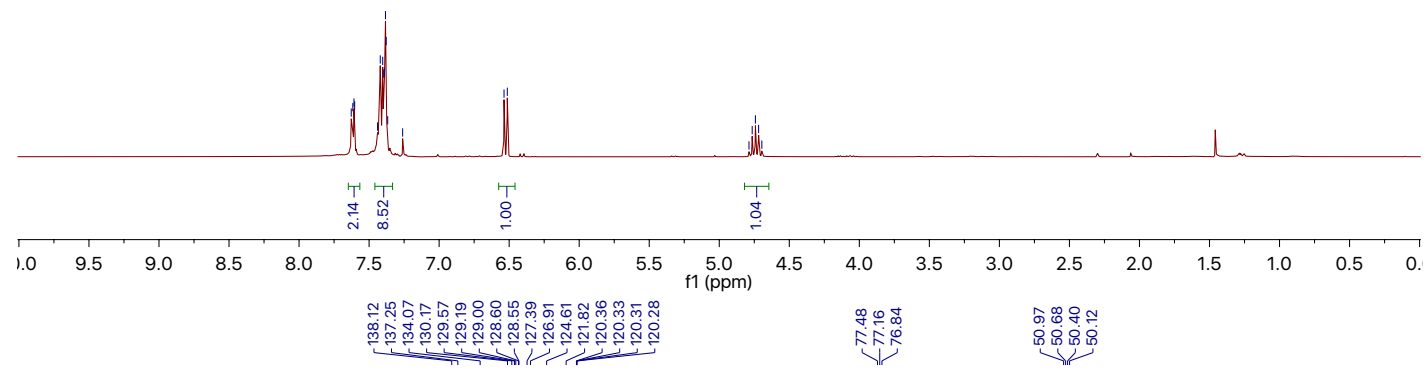

${ }^{13} \mathrm{C} \mathrm{NMR}\left(100 \mathrm{MHz}, \mathrm{CDCl}_{3}\right)$
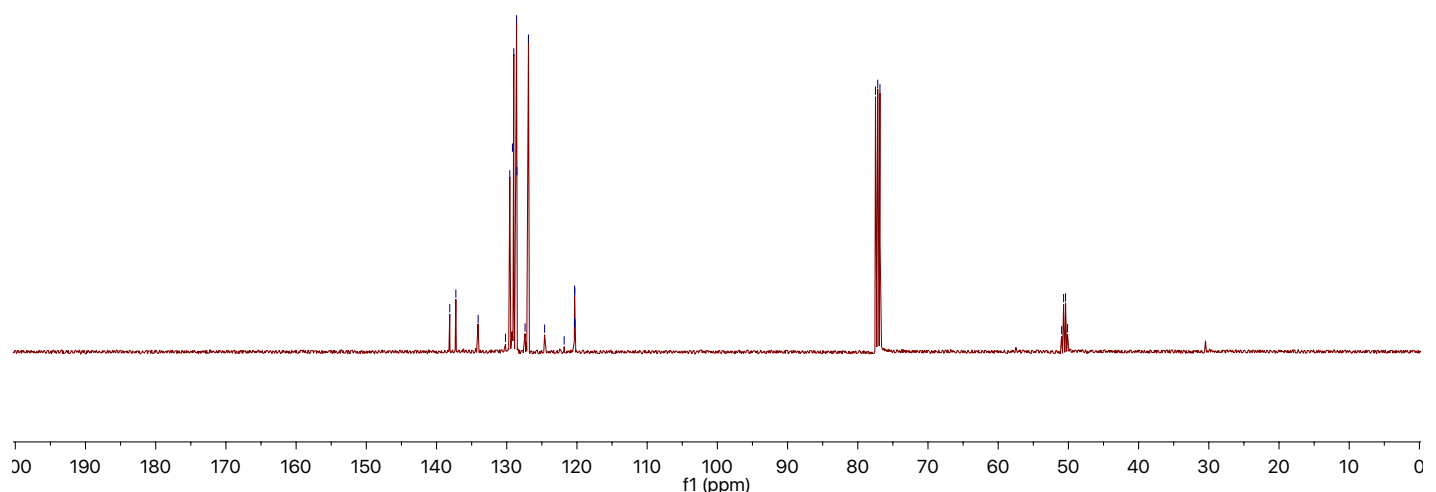
(Z)-1-bromo-4-(1-chloro-4,4,4-trifluoro-3-phenylbut-1-en-1-yl)benzene (5b)<smiles>FC(F)(F)C(/C=C(\Cl)c1ccc(Br)cc1)c1ccccc1</smiles>

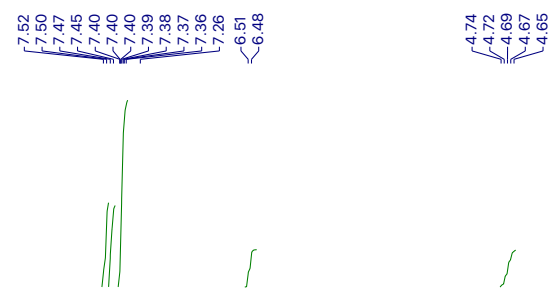

${ }^{1} \mathrm{H}$ NMR $\left(400 \mathrm{MHz}, \mathrm{CDCl}_{3}\right)$

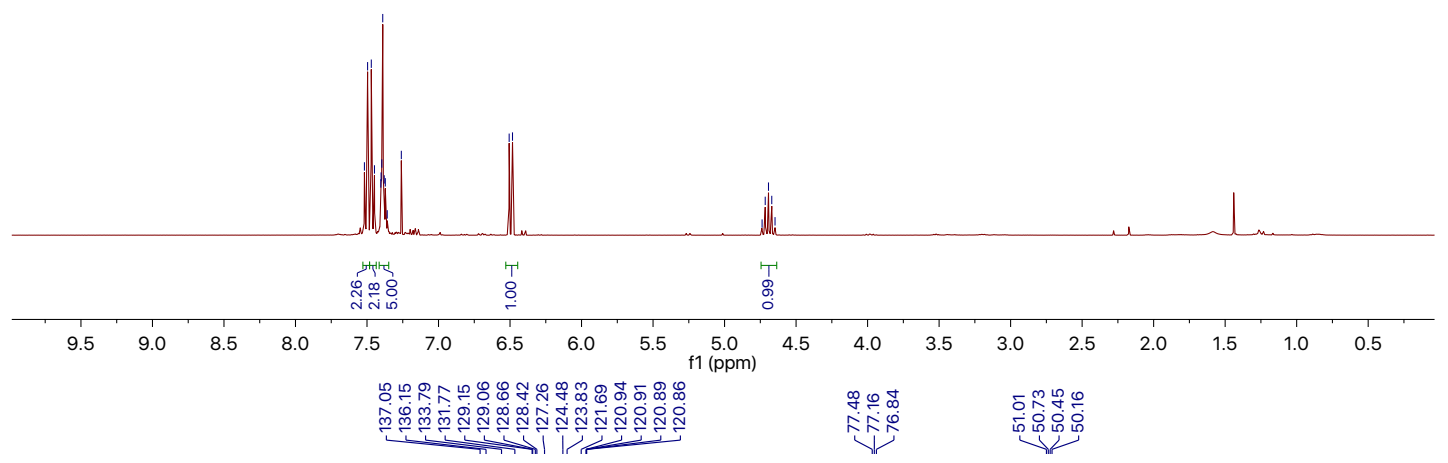

${ }^{13} \mathrm{C}$ NMR $\left(100 \mathrm{MHz}, \mathrm{CDCl}_{3}\right)$

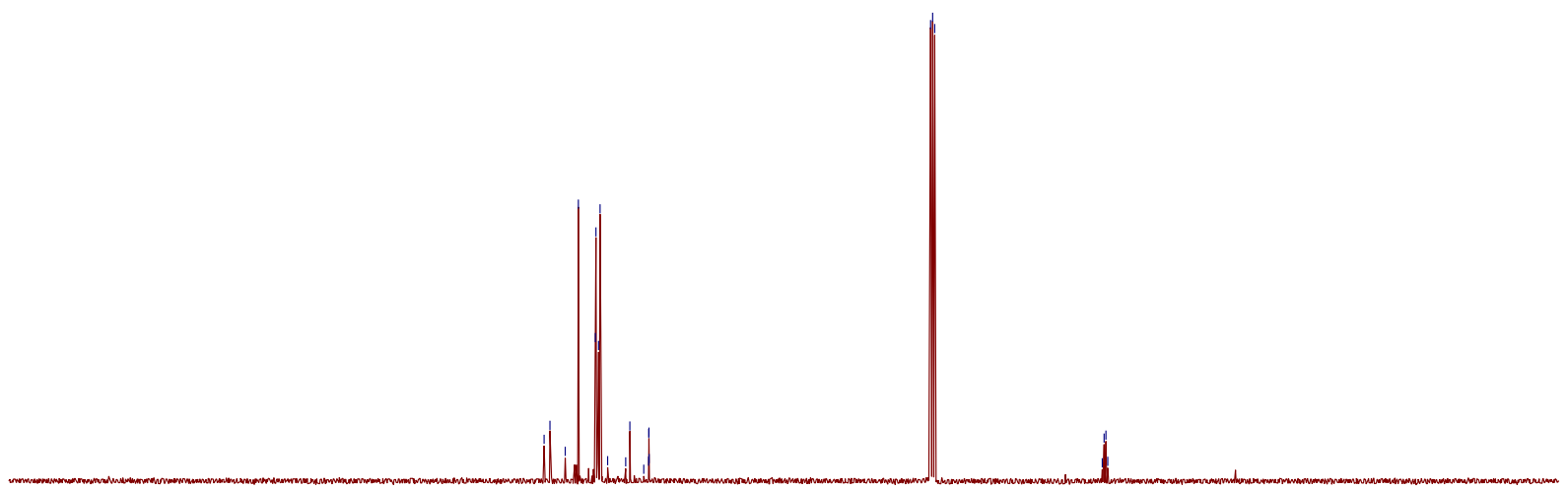

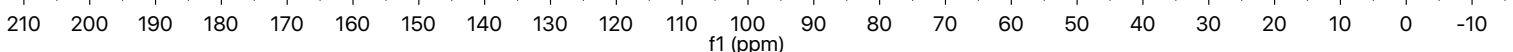




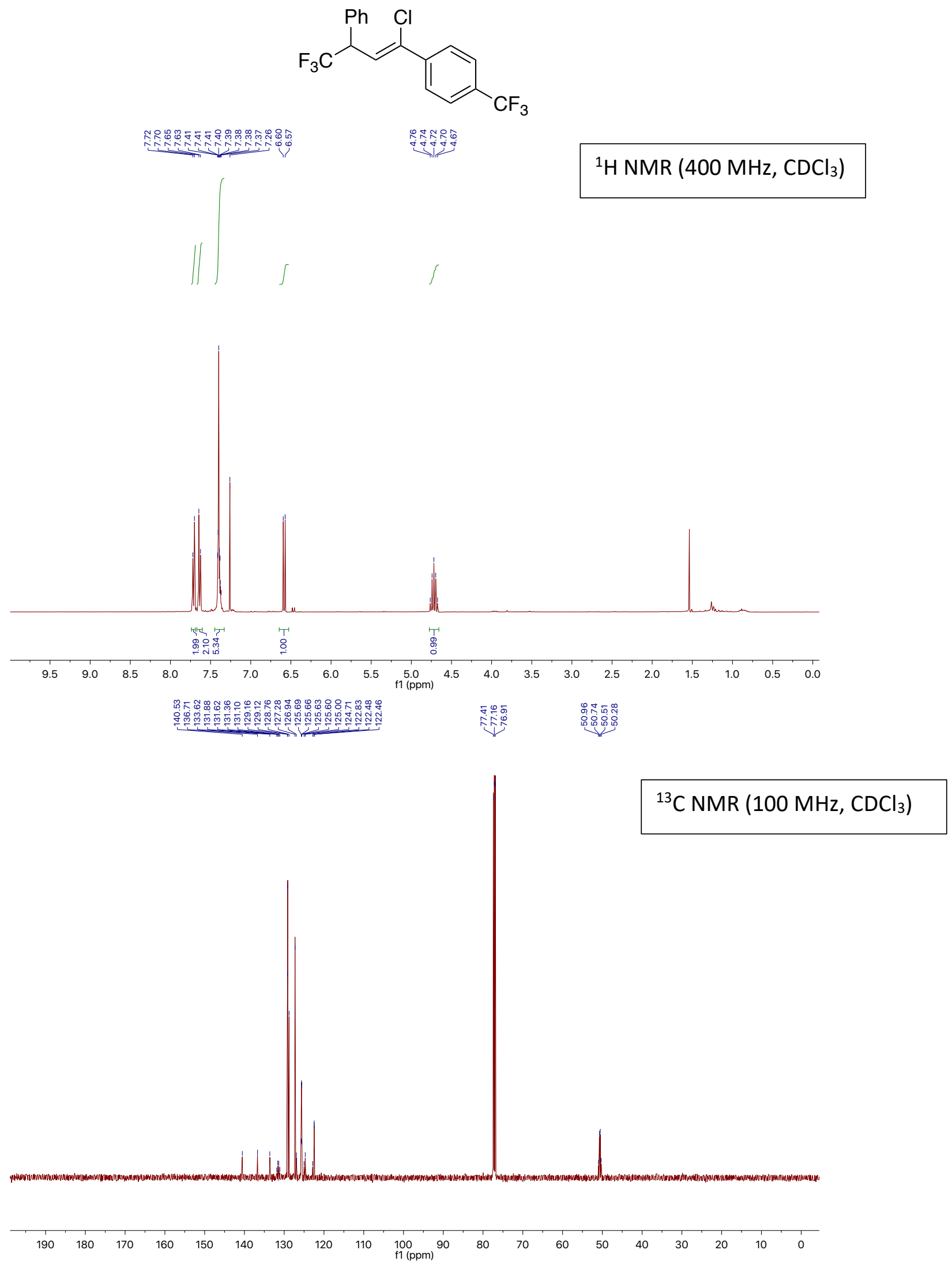


(Z)-4-(1-chloro-4,4,4-trifluoro-3-phenylbut-1-en-1-yl)benzonitrile (5d)<smiles>N#Cc1ccc(/C(Cl)=C/C(c2ccccc2)C(F)(F)F)cc1</smiles>

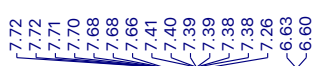

${ }^{1} \mathrm{H} \mathrm{NMR}\left(400 \mathrm{MHz}, \mathrm{CDCl}_{3}\right)$
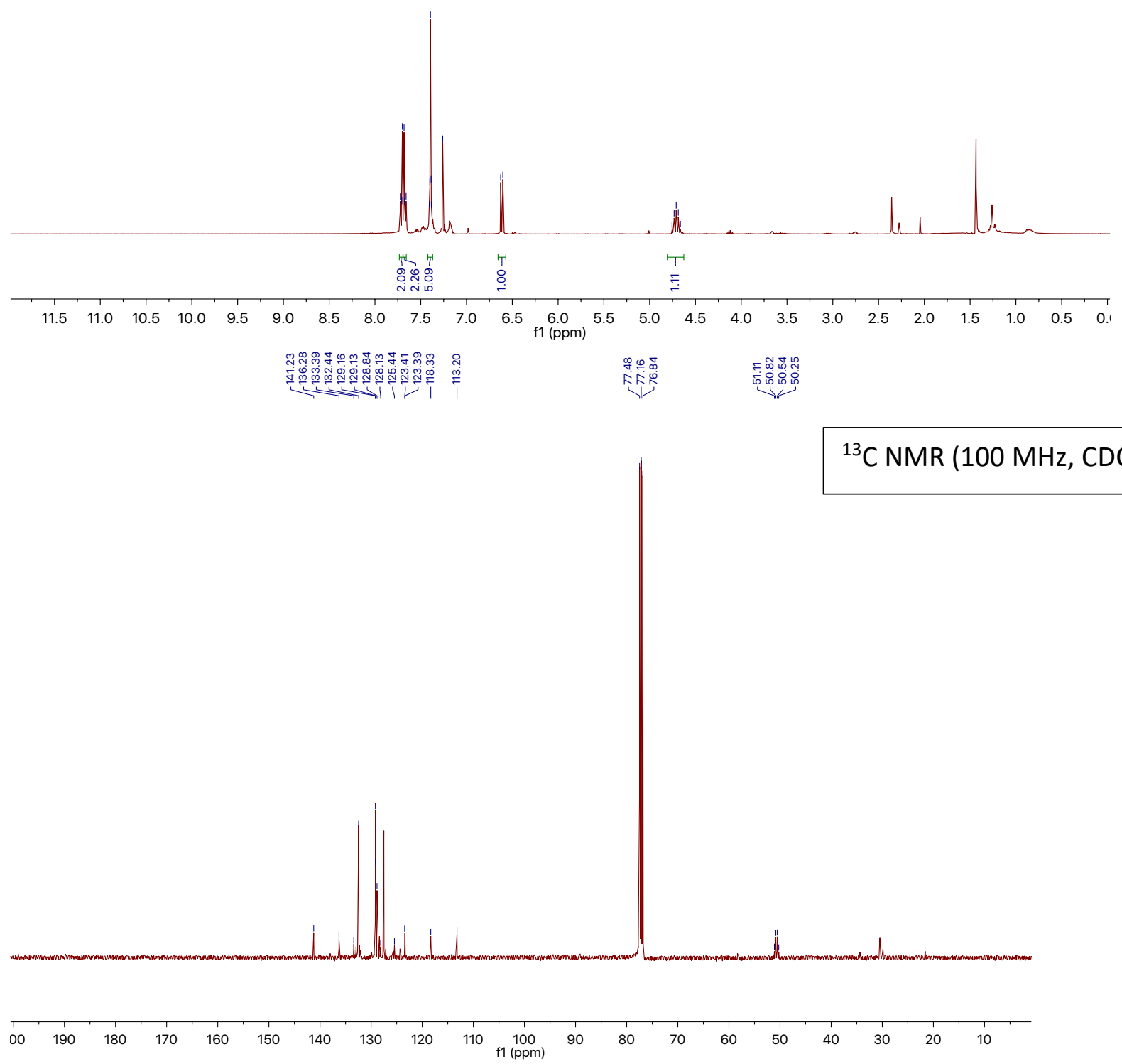

S29 

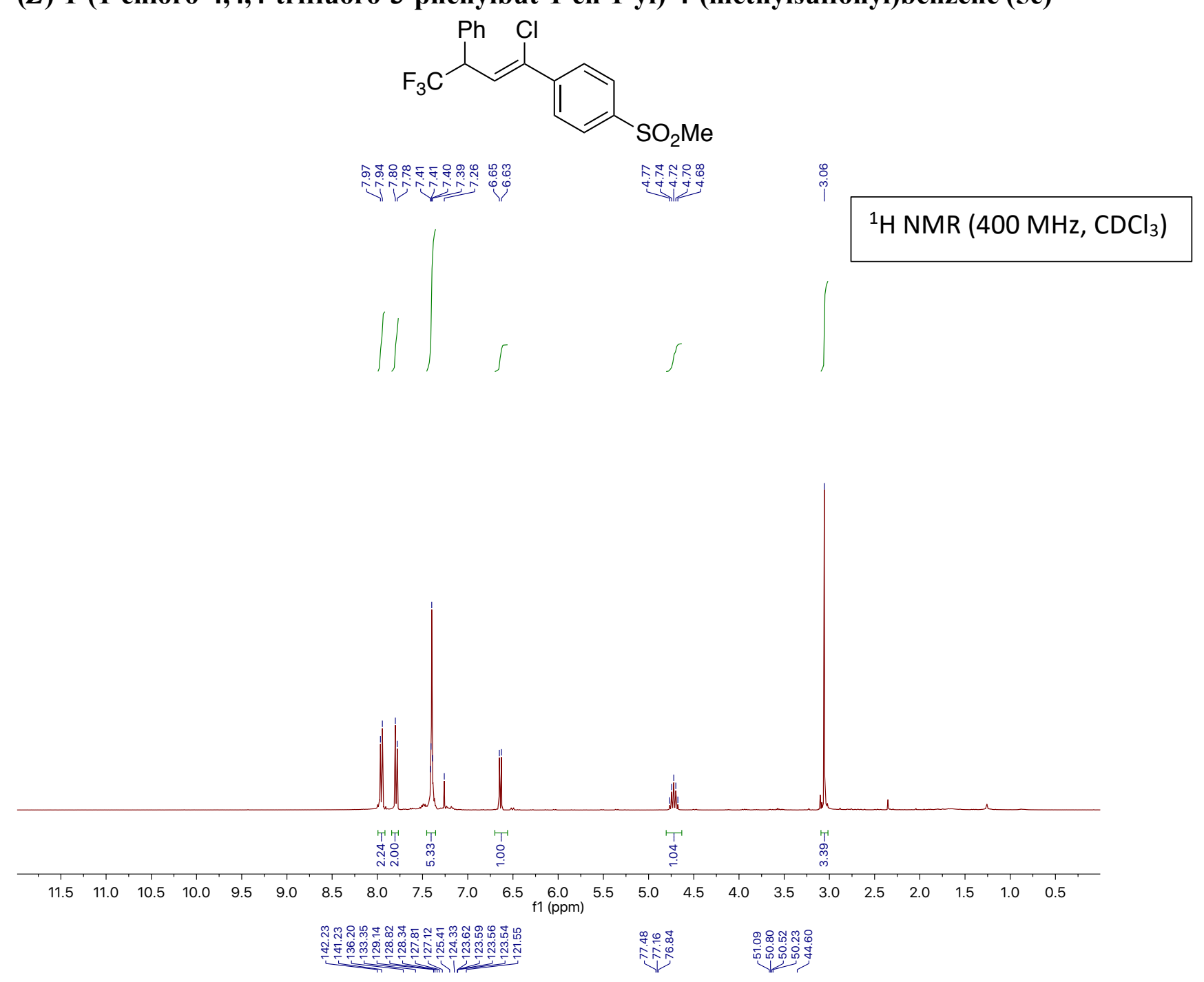

${ }^{13} \mathrm{C} \mathrm{NMR}\left(100 \mathrm{MHz}, \mathrm{CDCl}_{3}\right)$

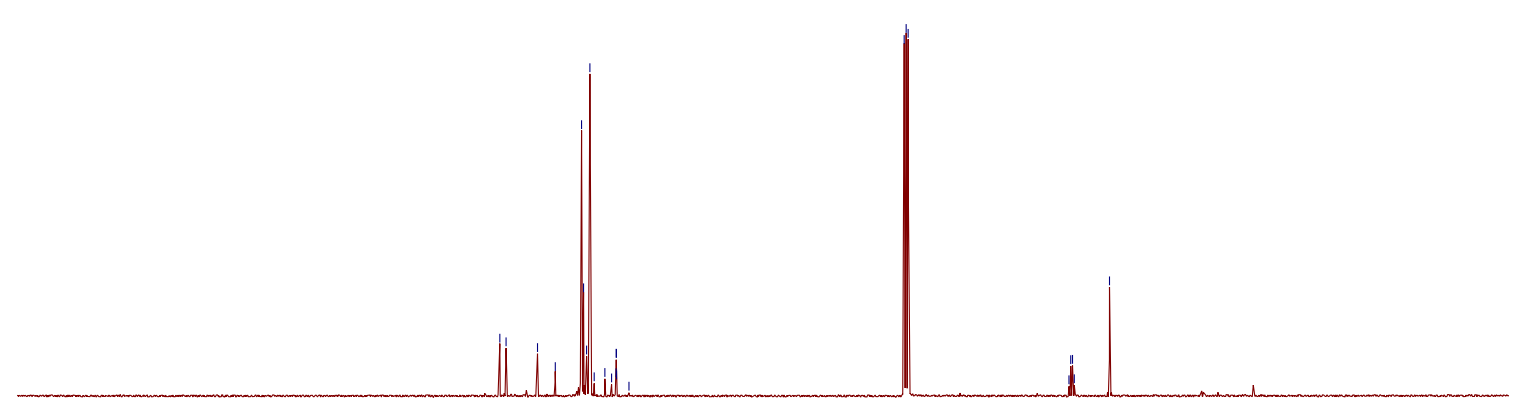

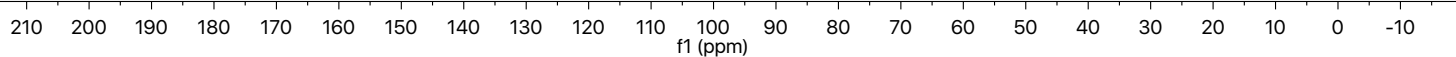


(Z)-1-(1-chloro-4,4,4-trifluoro-3-phenylbut-1-en-1-yl)-4-methylbenzene (5f)<smiles>Cc1ccc(/C(Cl)=C/C(c2ccccc2)C(F)(F)F)cc1</smiles>

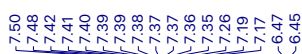

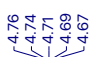

${ }^{1} \mathrm{H}$ NMR $\left(400 \mathrm{MHz}, \mathrm{CDCl}_{3}\right)$

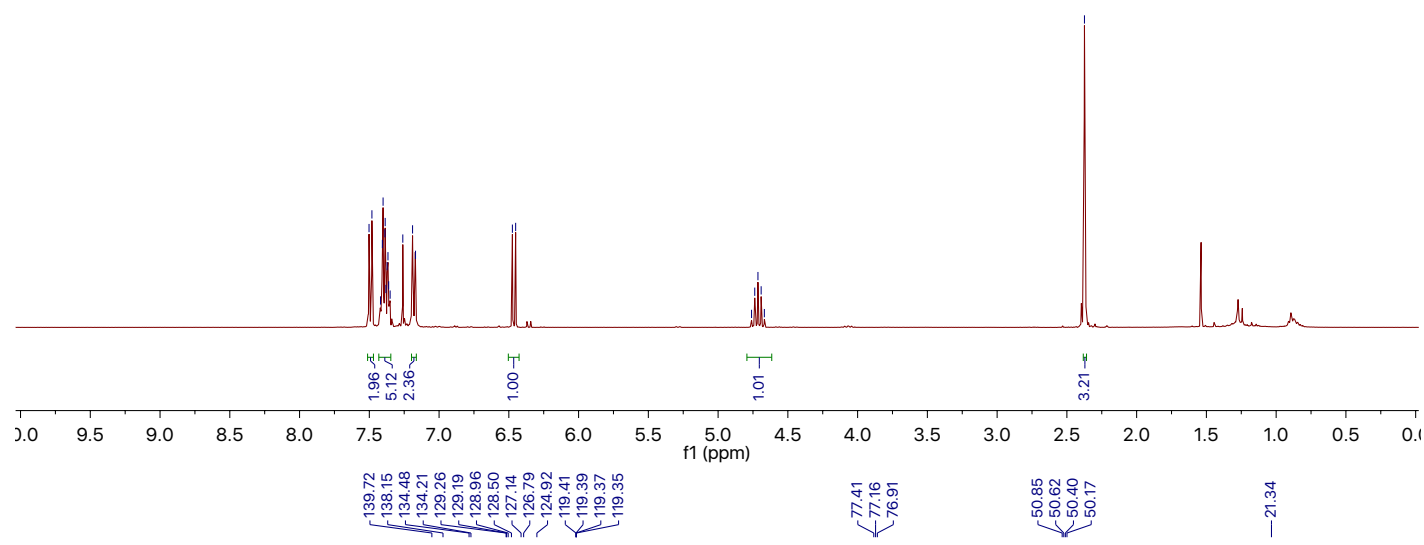

${ }^{13} \mathrm{C}$ NMR $\left(100 \mathrm{MHz}, \mathrm{CDCl}_{3}\right)$

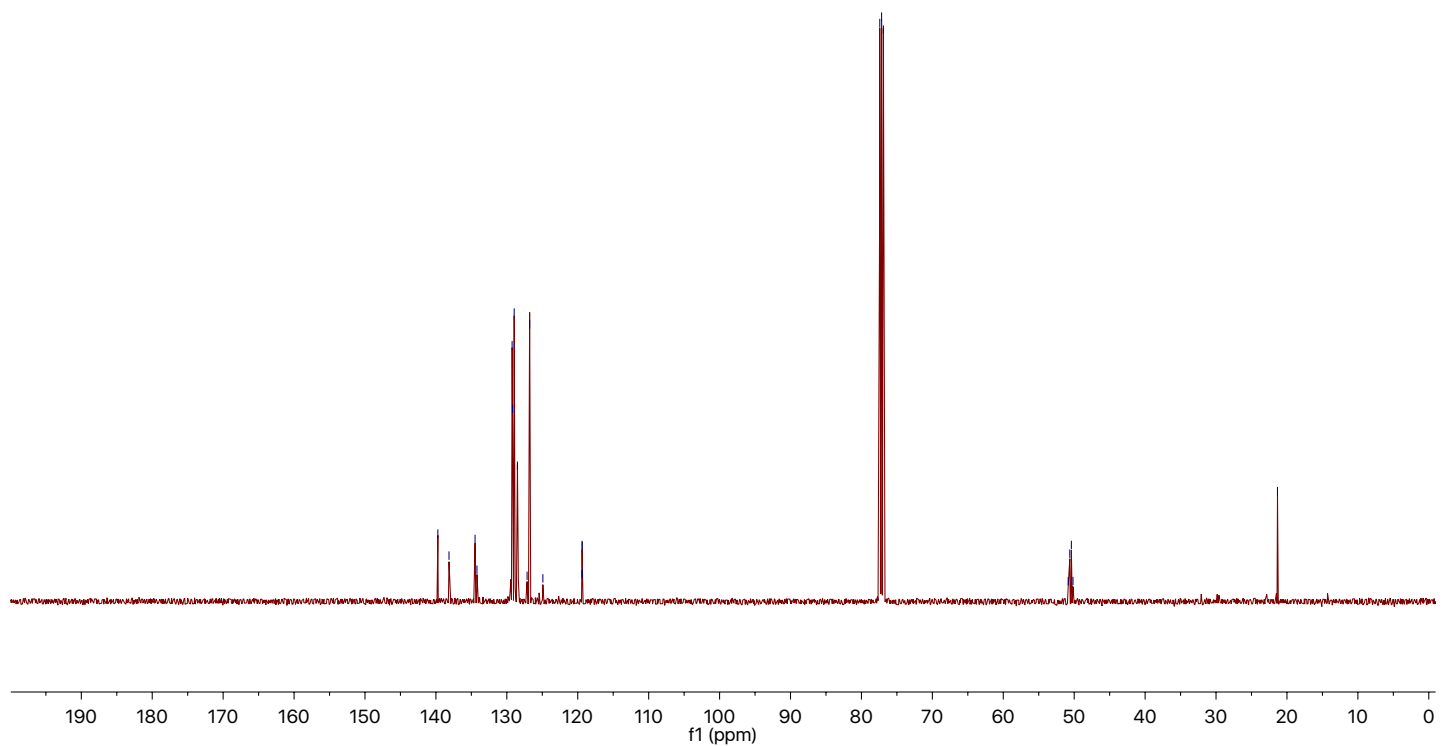


<smiles>FC(F)(F)C(/C=C(\Cl)c1ccc2ccccc2c1)c1ccccc1</smiles>

8.

${ }^{1} \mathrm{H}$ NMR $\left(400 \mathrm{MHz}, \mathrm{CDCl}_{3}\right)$

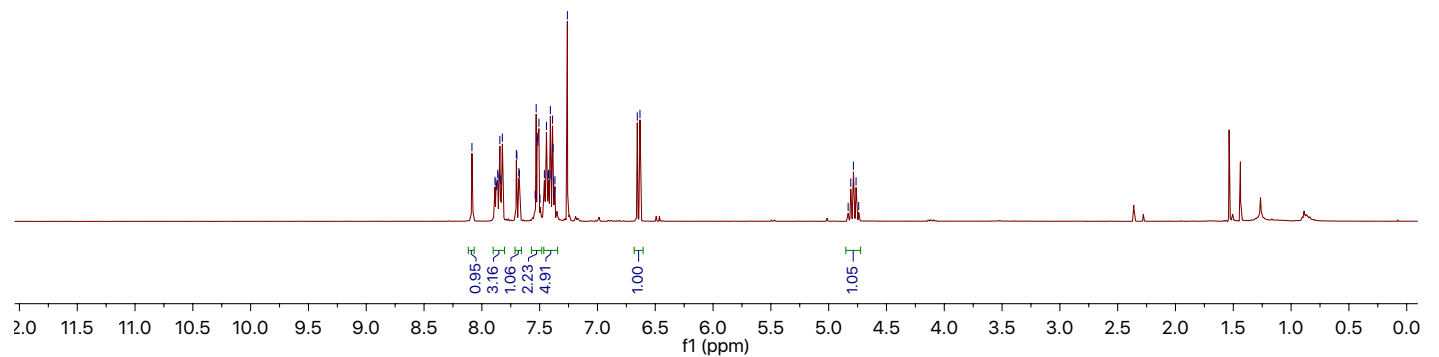

จำ

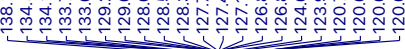
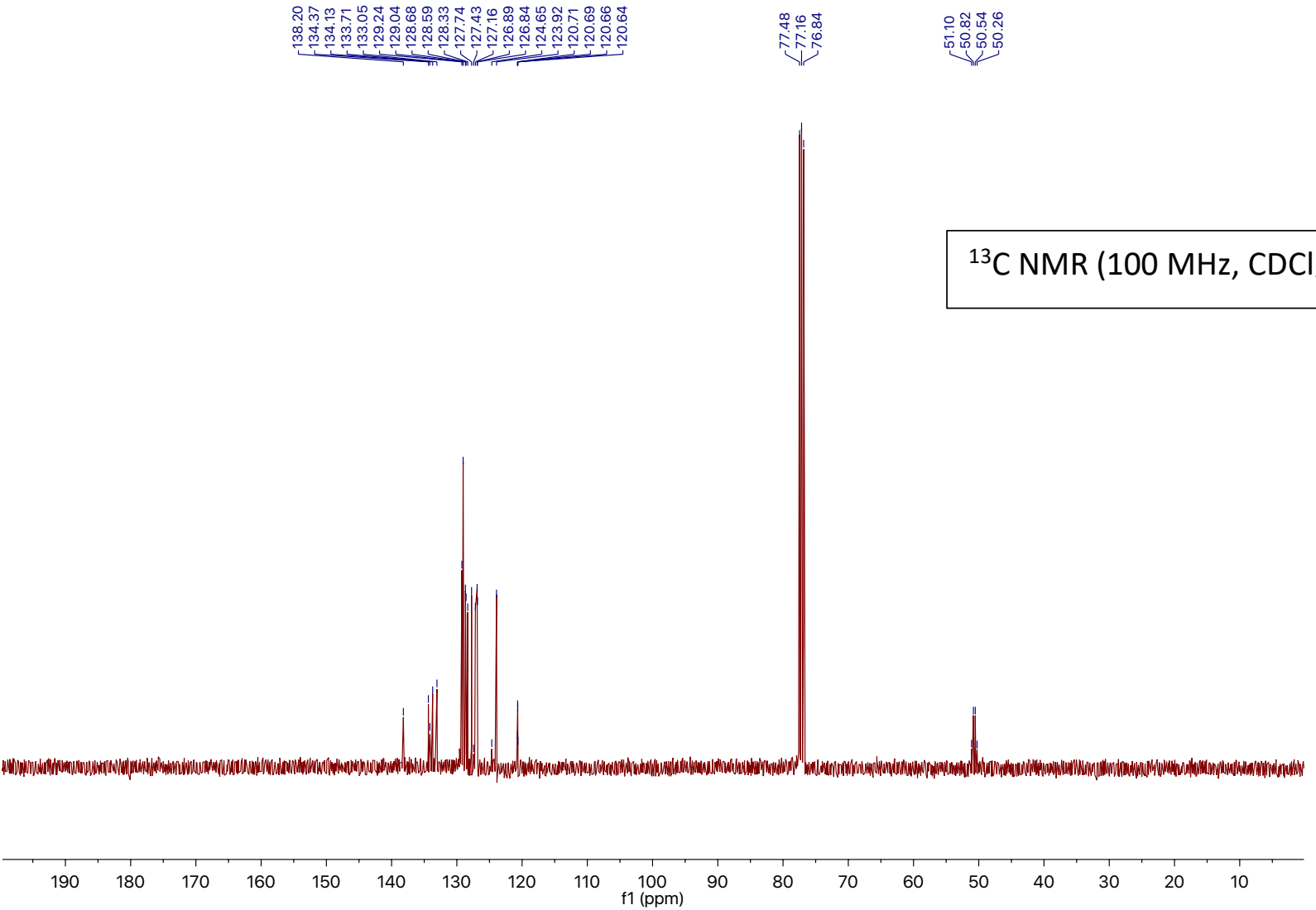
(Z)-(1-chloro-4,4,4-trifluorobut-1-en-1-yl)benzene (5j)
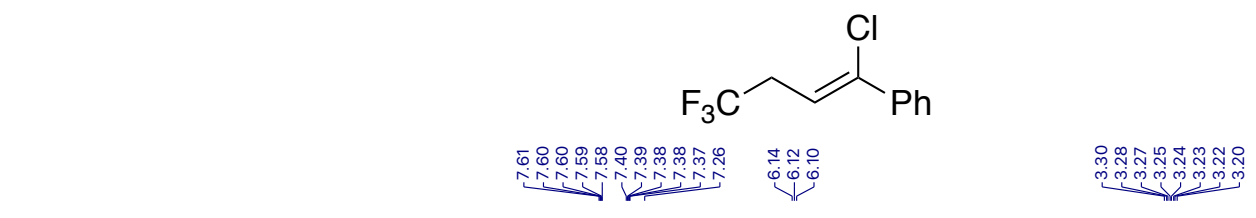

${ }^{1} \mathrm{H} \mathrm{NMR}\left(400 \mathrm{MHz}, \mathrm{CDCl}_{3}\right)$

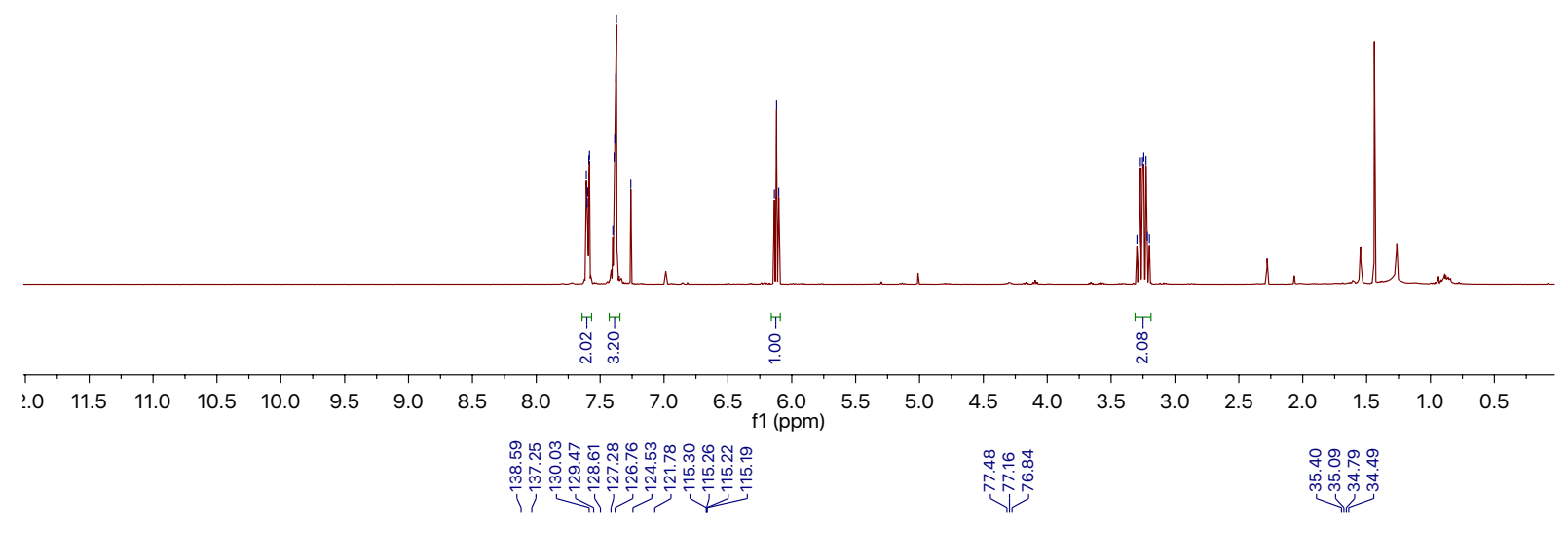

${ }^{13} \mathrm{C} \mathrm{NMR}\left(100 \mathrm{MHz}, \mathrm{CDCl}_{3}\right)$

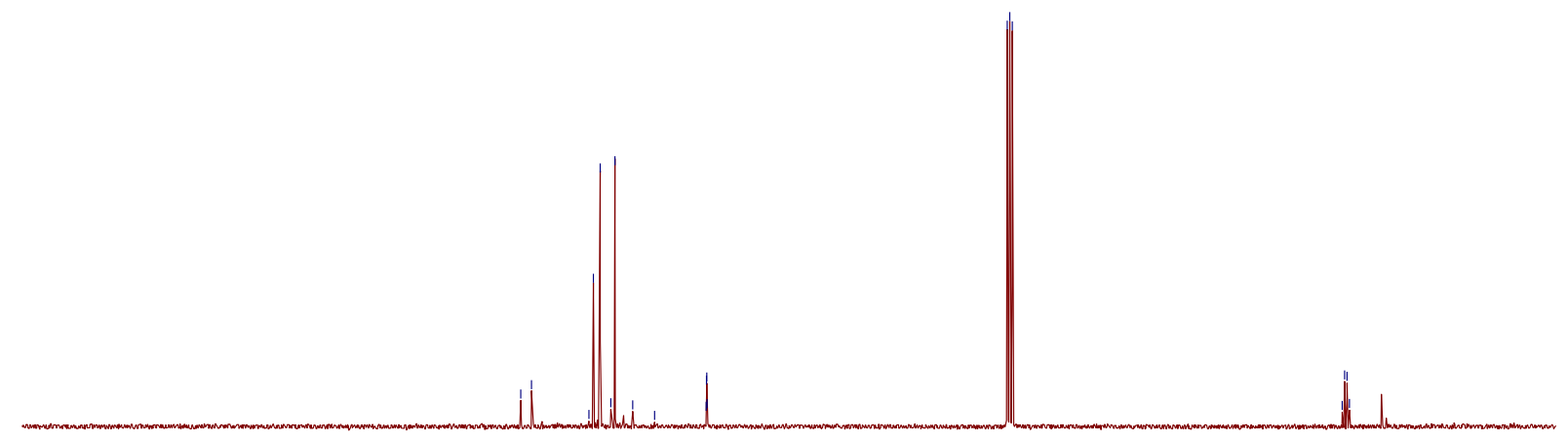

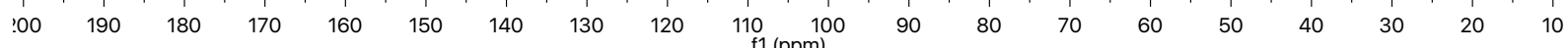


(Z)-(1-chloro-4,4,4-trifluoro-3-methylbut-1-en-1-yl)benzene (5k)

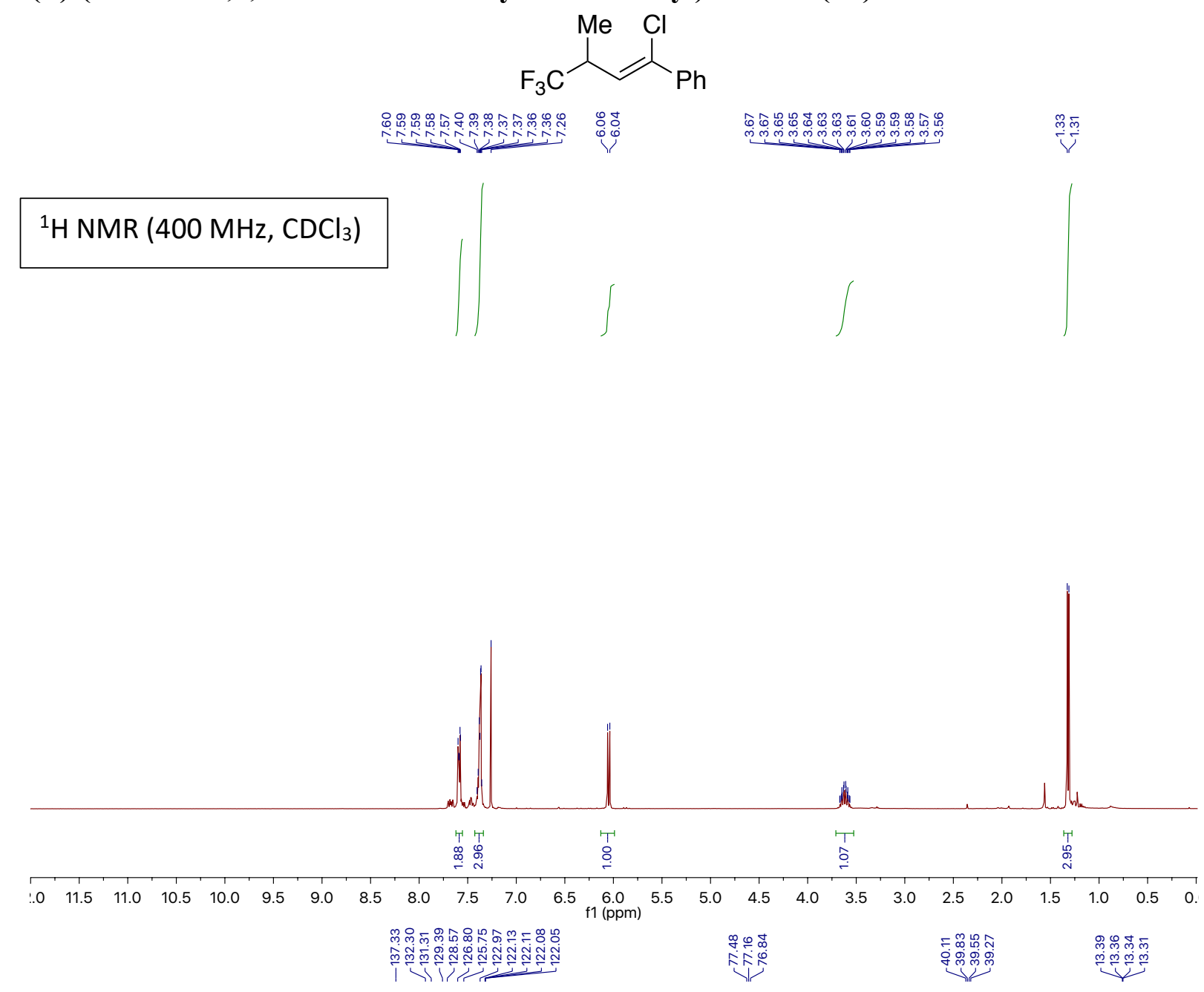

${ }^{13} \mathrm{C} \mathrm{NMR}\left(100 \mathrm{MHz}, \mathrm{CDCl}_{3}\right)$

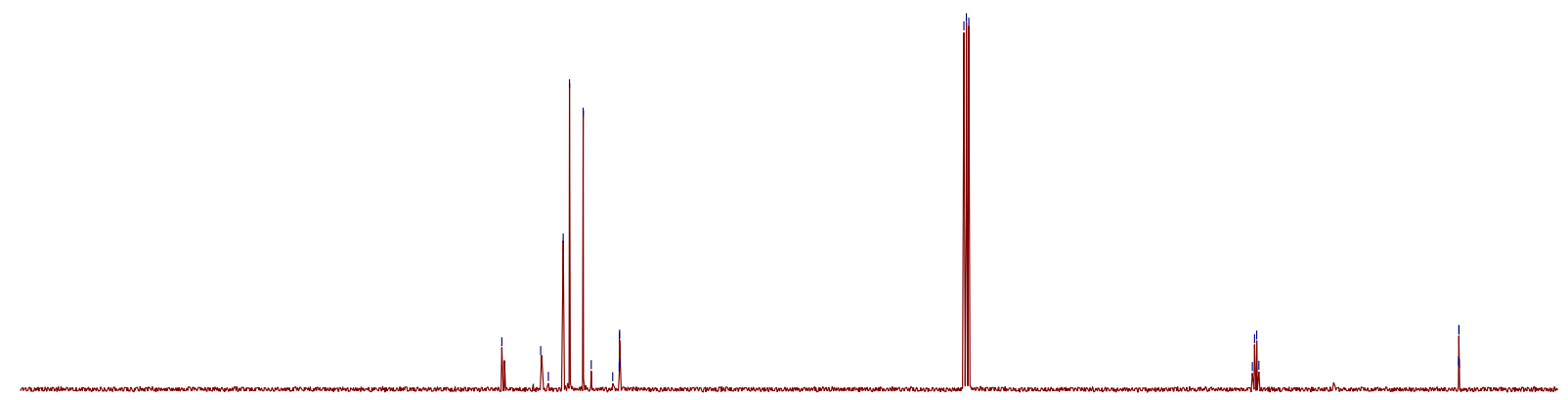

$\begin{array}{lllllllllllllllllll}190 & 180 & 170 & 160 & 150 & 140 & 130 & 120 & 110 & 100 & 90 & 80 & 70 & 60 & 50 & 40 & 30 & 20 & 10\end{array}$ 
(Z)-1-chloro-4-(4-chloro-1,1,1-trifluoro-4-phenylbut-3-en-2-yl)benzene (5l)<smiles>FC(F)(F)/C(Cl)=C/c1ccc(Cl)cc1</smiles>

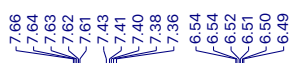

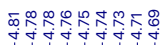

${ }^{1} \mathrm{H} \mathrm{NMR}\left(400 \mathrm{MHz}, \mathrm{CDCl}_{3}\right)$

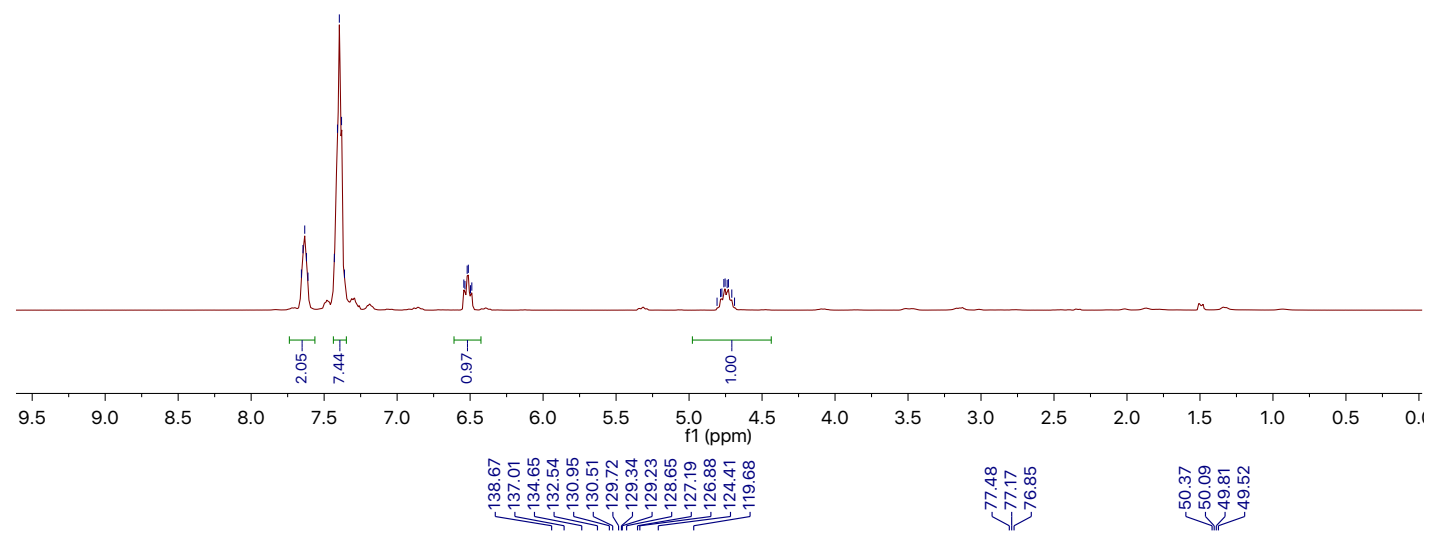

${ }^{13} \mathrm{C} \mathrm{NMR}\left(100 \mathrm{MHz}, \mathrm{CDCl}_{3}\right)$ 
(Z)-1-(4-chloro-1,1,1-trifluoro-4-phenylbut-3-en-2-yl)-4-(trifluoromethyl)benzene (5m)<smiles>FC(F)(F)c1ccc(C(/C=C(\Cl)c2ccccc2)C(F)(F)F)cc1</smiles>

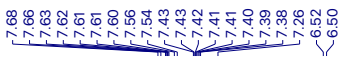

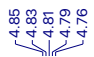

${ }^{1} \mathrm{H} \mathrm{NMR}\left(400 \mathrm{MHz}, \mathrm{CDCl}_{3}\right)$

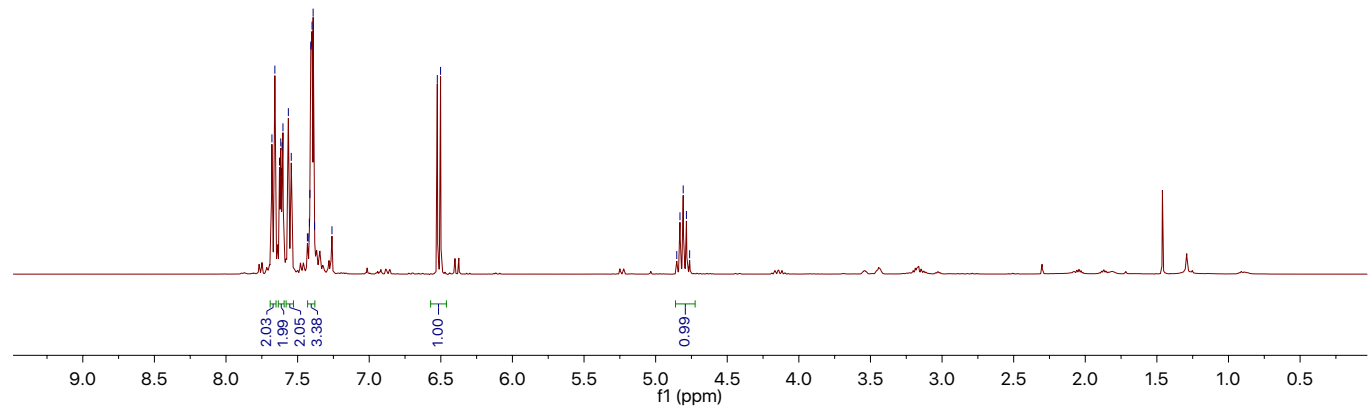

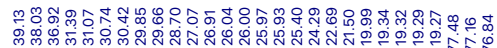

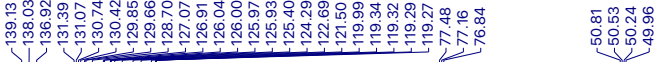

$\left.{ }^{13} \mathrm{C} \mathrm{NMR} \mathrm{(100} \mathrm{MHz,} \mathrm{CDCl}_{3}\right)$

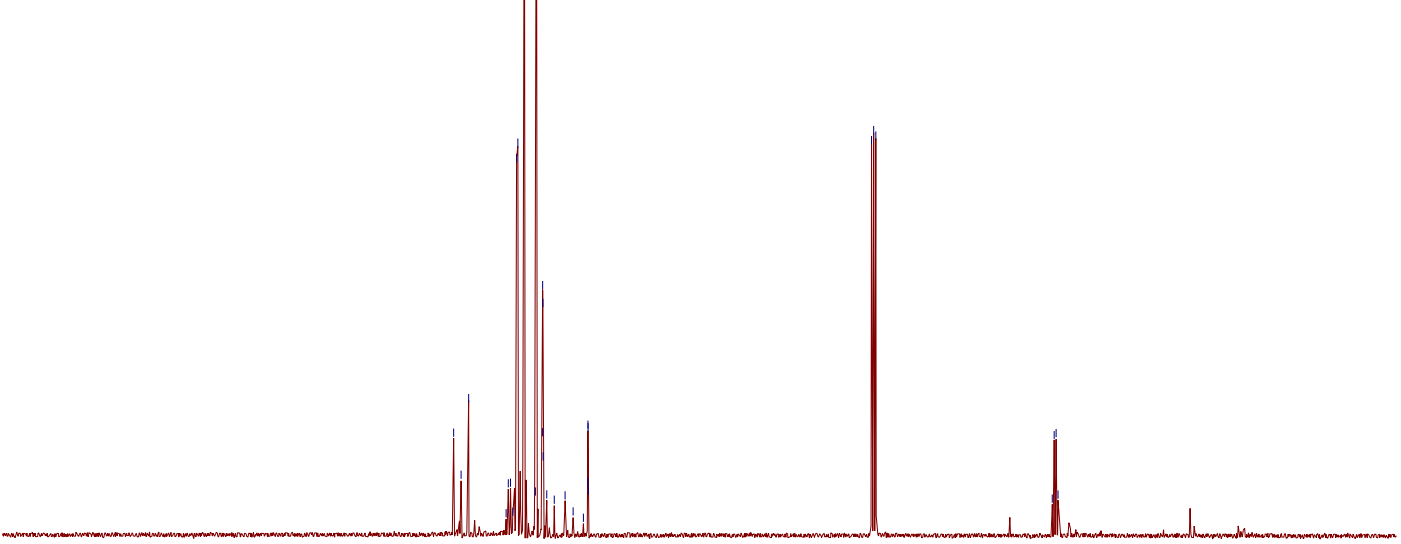

$\begin{array}{llllllllll}200 & 190 & 180 & 170 & 160 & 150 & 140 & 130 & 120 & 110\end{array}$ 
(Z)-(1-chloro-3-(phenylsulfonyl)prop-1-ene-1,3-diyl)dibenzene ((Z)-5n)
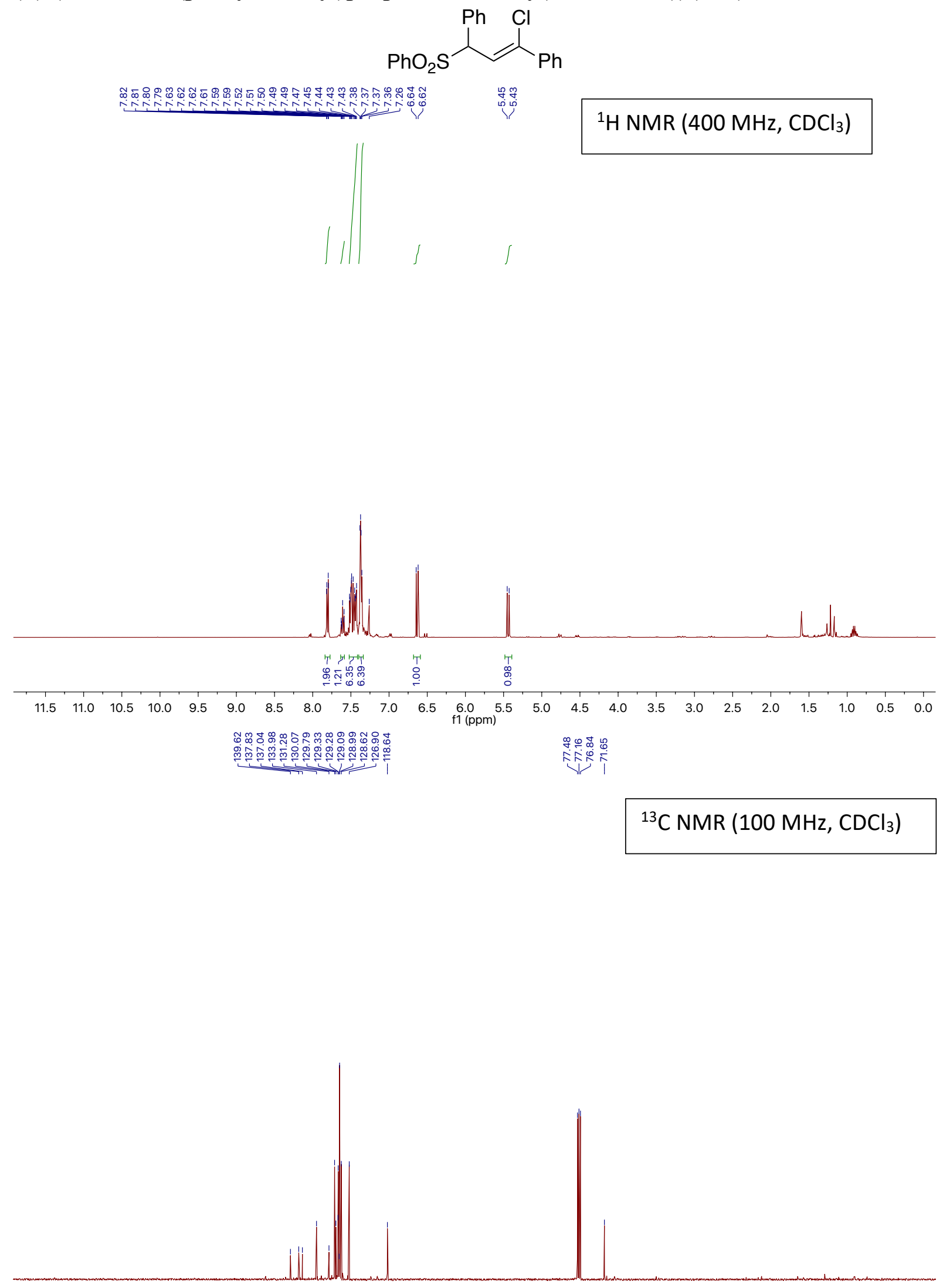

$\begin{array}{llllllllllllllllllll}190 & 180 & 170 & 160 & 150 & 140 & 130 & 120 & 110 & 100 & 90 & 80 & 70 & 60 & 50 & 40 & 30 & 20 & 10 & \mathrm{C}\end{array}$ 
(E)-(1-chloro-3-(phenylsulfonyl)prop-1-ene-1,3-diyl)dibenzene ((E)-5n)

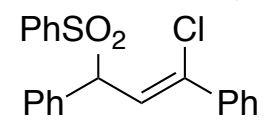

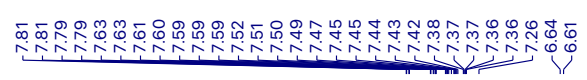

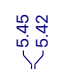

${ }^{1} \mathrm{H} \mathrm{NMR}\left(400 \mathrm{MHz}, \mathrm{CDCl}_{3}\right)$
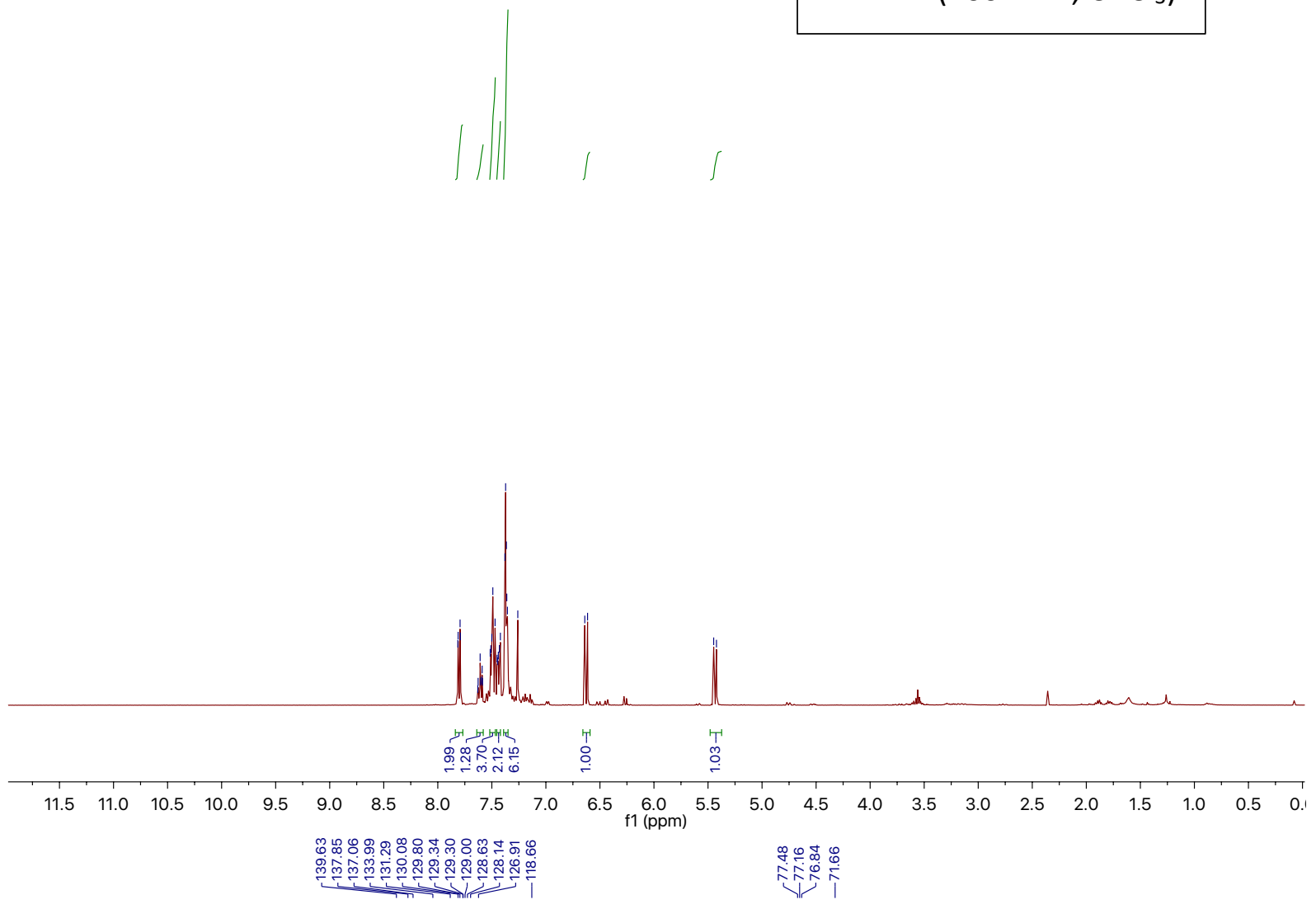

${ }^{13} \mathrm{C} \mathrm{NMR}\left(100 \mathrm{MHz}, \mathrm{CDCl}_{3}\right)$
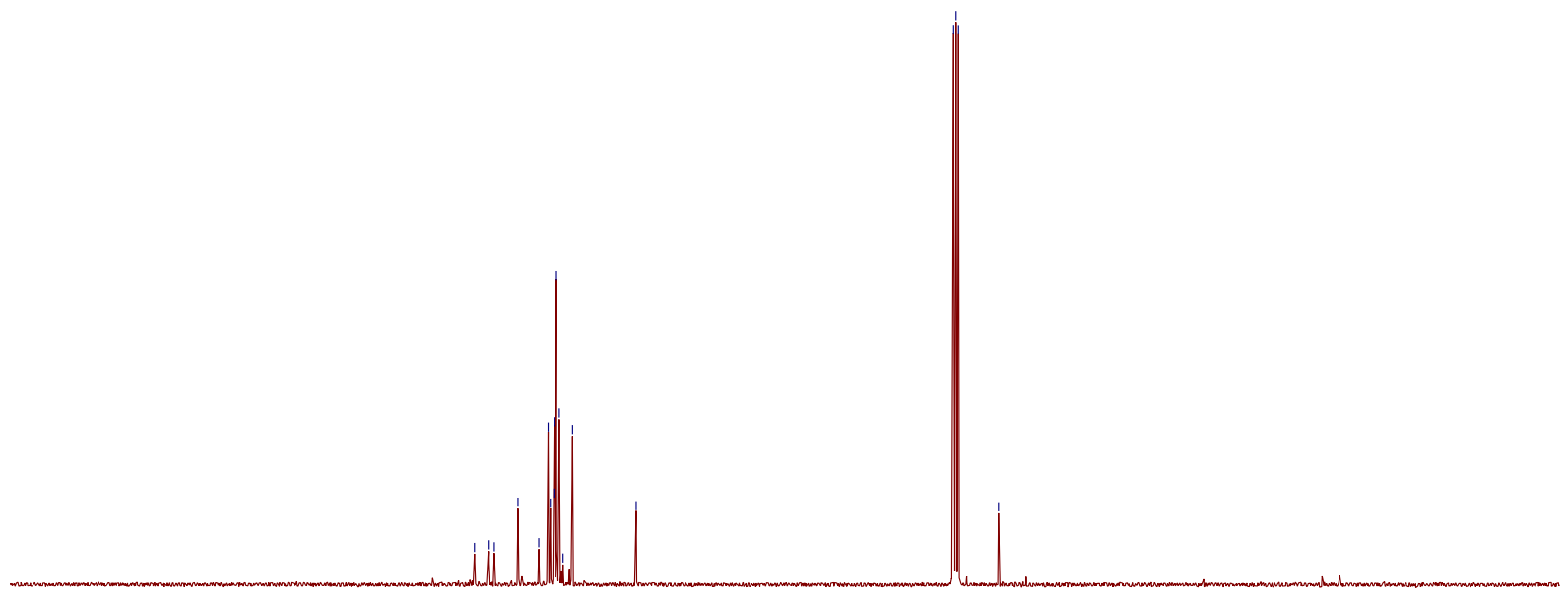

190

180

160

140

100
$f 1(p p m)$

S38 
(Z)-(1-bromo-4,4,4-trifluorobut-1-ene-1,3-diyl)dibenzene (6a)
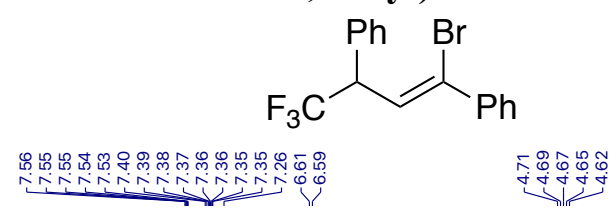

${ }^{1} \mathrm{H} \mathrm{NMR}\left(400 \mathrm{MHz}, \mathrm{CDCl}_{3}\right)$

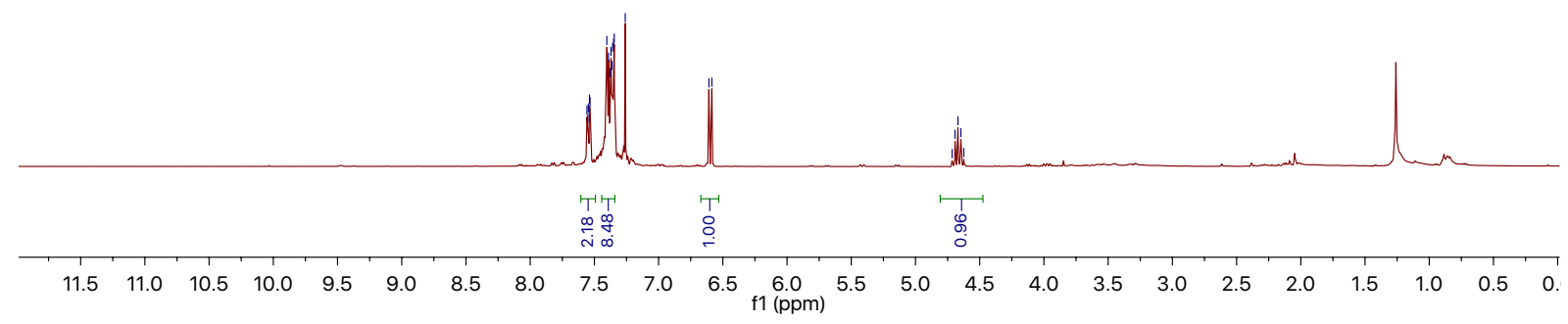

ํ.

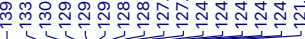

${ }^{13} \mathrm{C} \mathrm{NMR}\left(100 \mathrm{MHz}, \mathrm{CDCl}_{3}\right)$

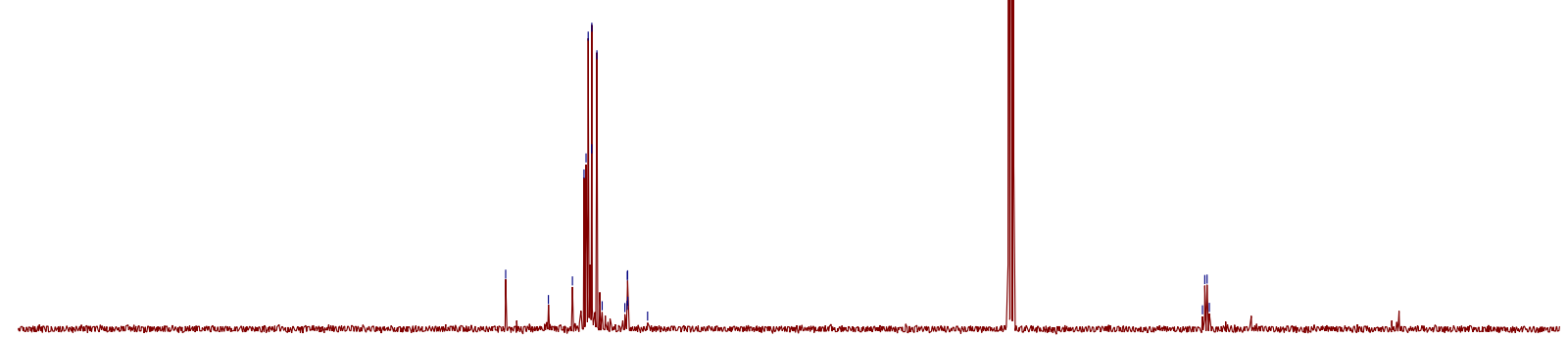

$\begin{array}{lllllllllllllllllll}190 & 180 & 170 & 160 & 150 & 140 & 130 & 120 & \begin{array}{c}110 \\ \mathrm{f} 1(\mathrm{ppm})\end{array} & 90 & 80 & 70 & 60 & 50 & 40 & 30 & 20 & 11\end{array}$ 
(Z)-1-(1-bromo-4,4,4-trifluoro-3-phenylbut-1-en-1-yl)-4-(trifluoromethyl)benzene (6b)

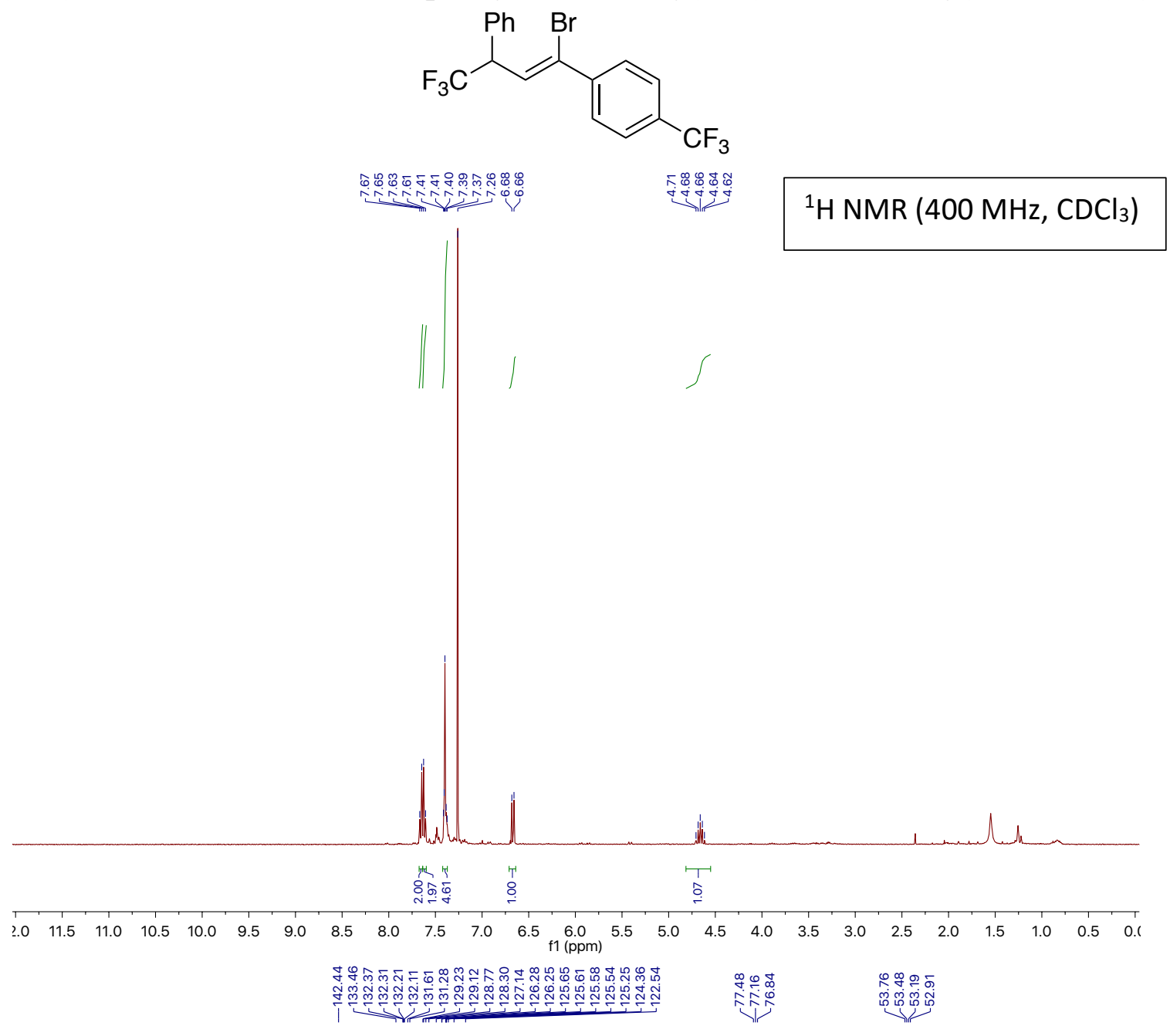

${ }^{13} \mathrm{C} \mathrm{NMR}\left(100 \mathrm{MHz}, \mathrm{CDCl}_{3}\right)$

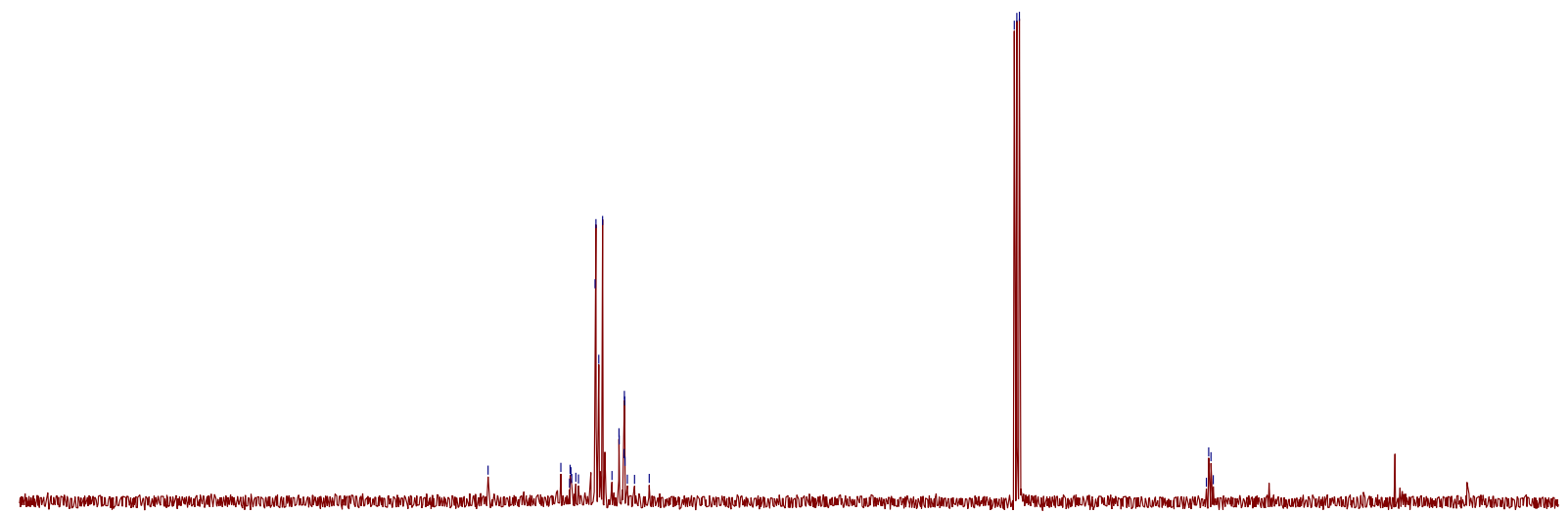

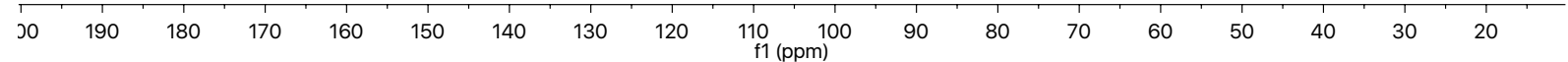


Z)-(1-bromo-3-(phenylsulfonyl)prop-1-ene-1,3-diyl)dibenzene (6c)<smiles>O=C(Oc1ccccc1)/C(Br)=C/C(c1ccccc1)c1ccccc1</smiles>

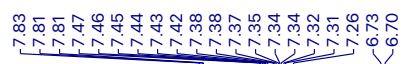

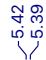

${ }^{1} \mathrm{H} \mathrm{NMR}\left(400 \mathrm{MHz}, \mathrm{CDCl}_{3}\right)$

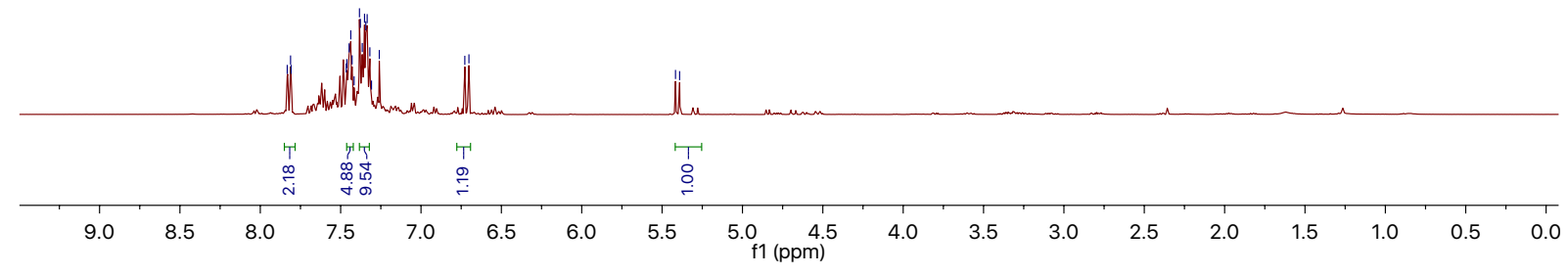

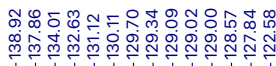

$\left.{ }^{13} \mathrm{C} \mathrm{NMR} \mathrm{(100} \mathrm{MHz,} \mathrm{CDCl}_{3}\right)$

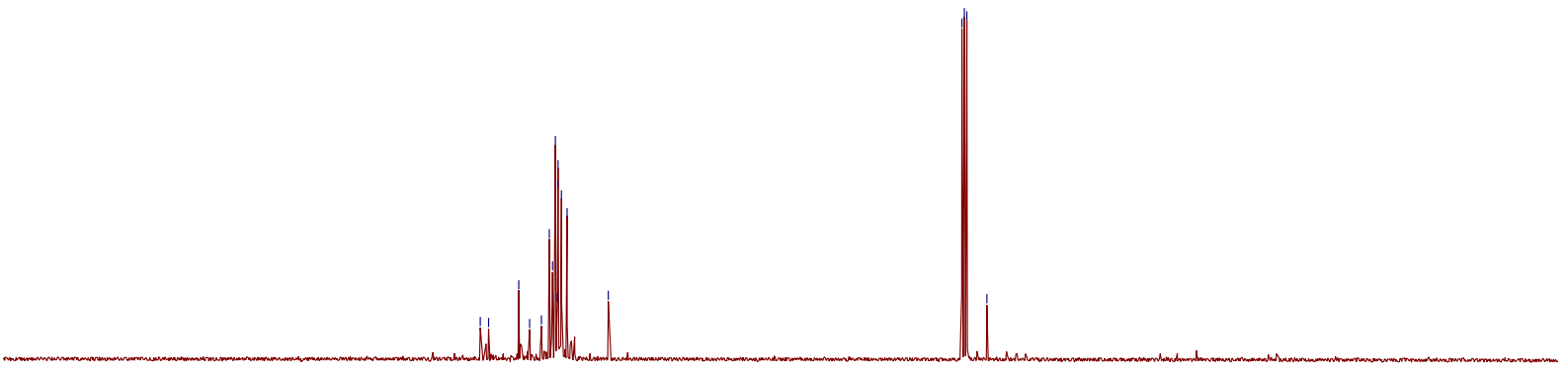


(Z)-(1-fluoro-4,4,4-trifluorobut-1-ene-1,3-diyl)dibenzene (7a)

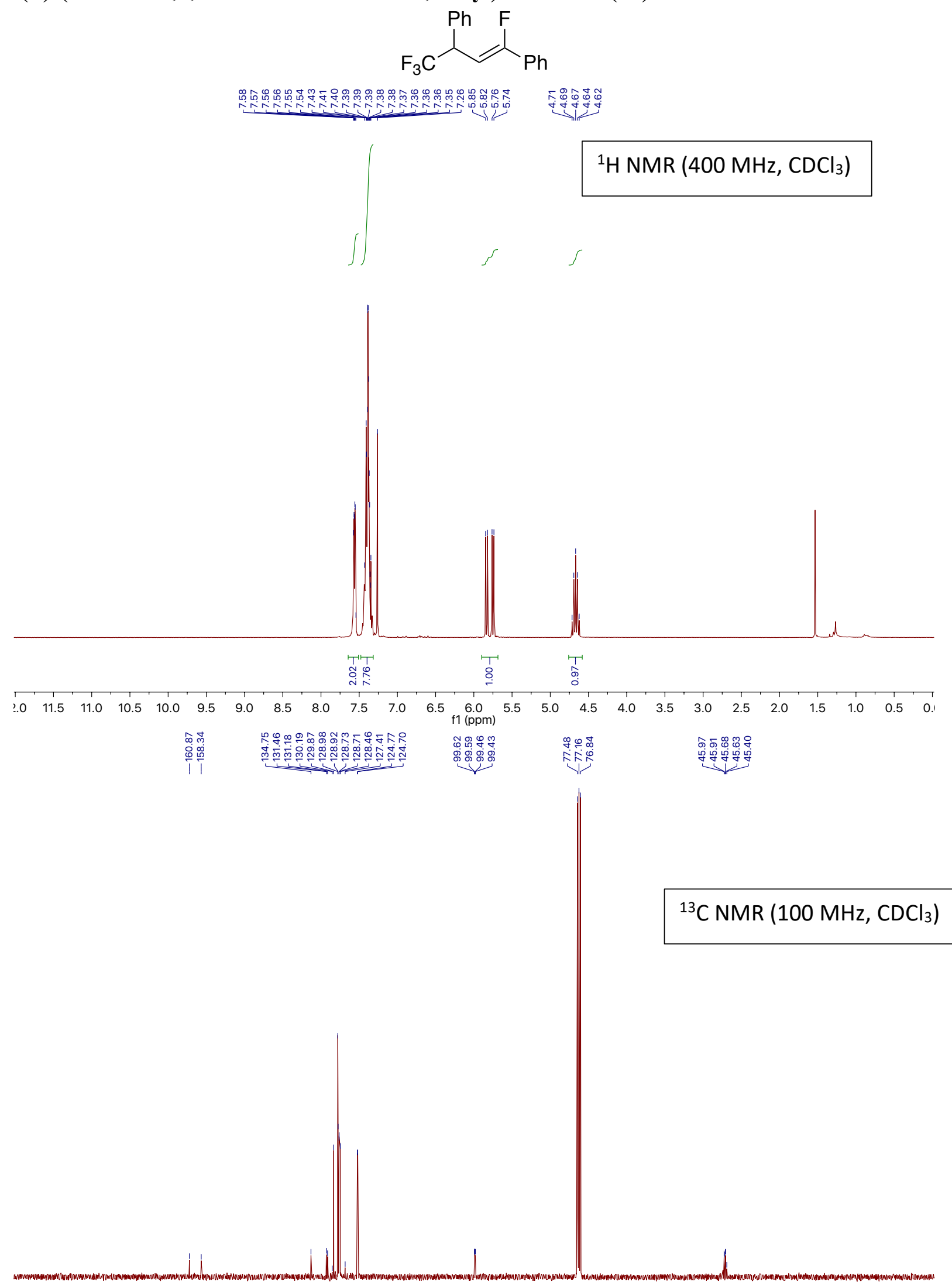

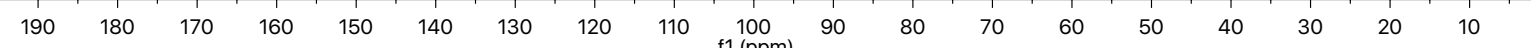


(Z)-1-(1-fluoro-4,4,4-trifluoro-3-phenylbut-1-en-1-yl)-4-(trifluoromethyl)benzene (7b)<smiles>FC(=CC(c1ccccc1)C(F)(F)F)c1ccc(C(F)(F)F)cc1</smiles>

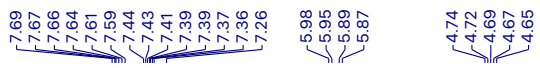
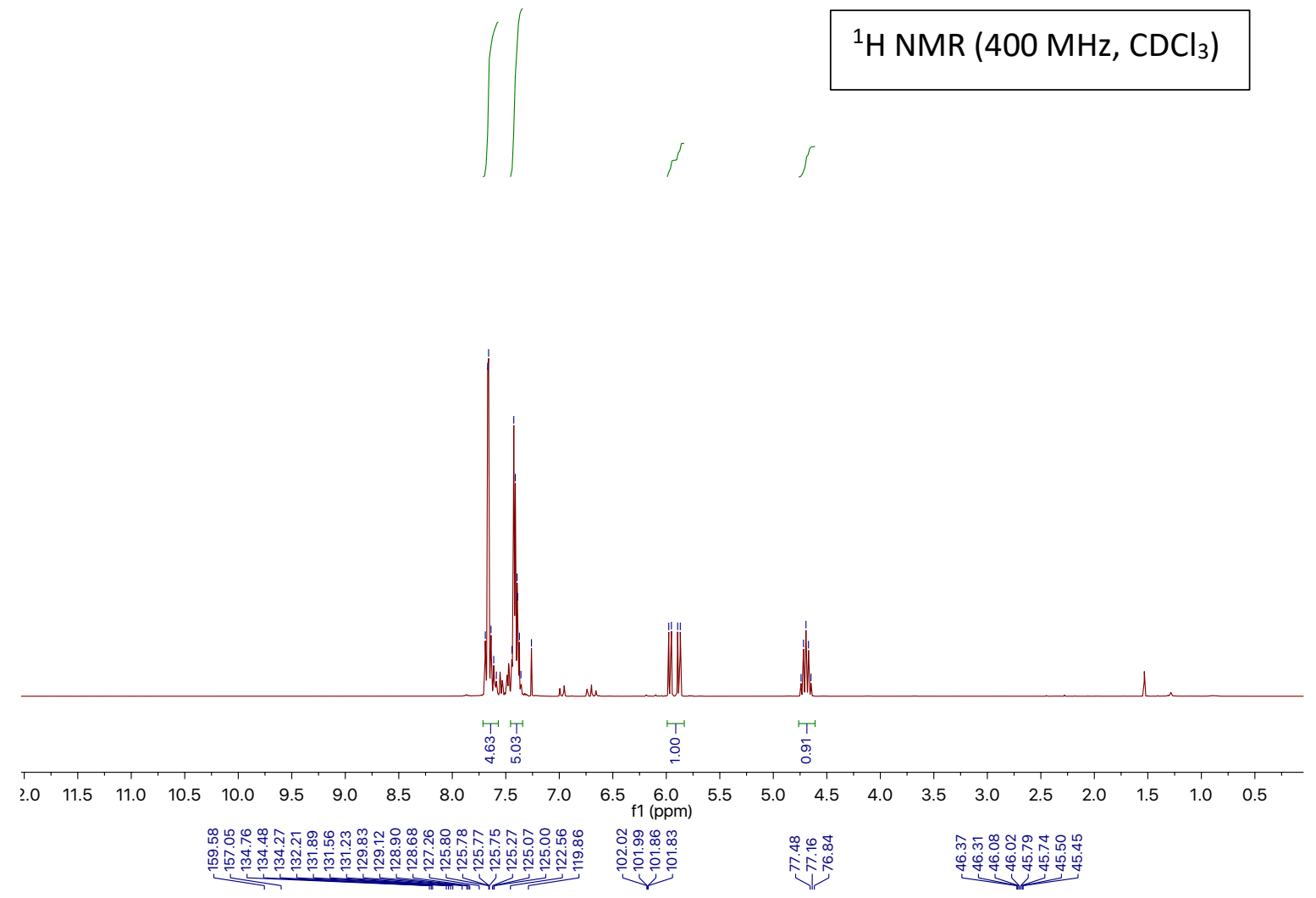

${ }^{13} \mathrm{C} \mathrm{NMR}\left(100 \mathrm{MHz}, \mathrm{CDCl}_{3}\right)$

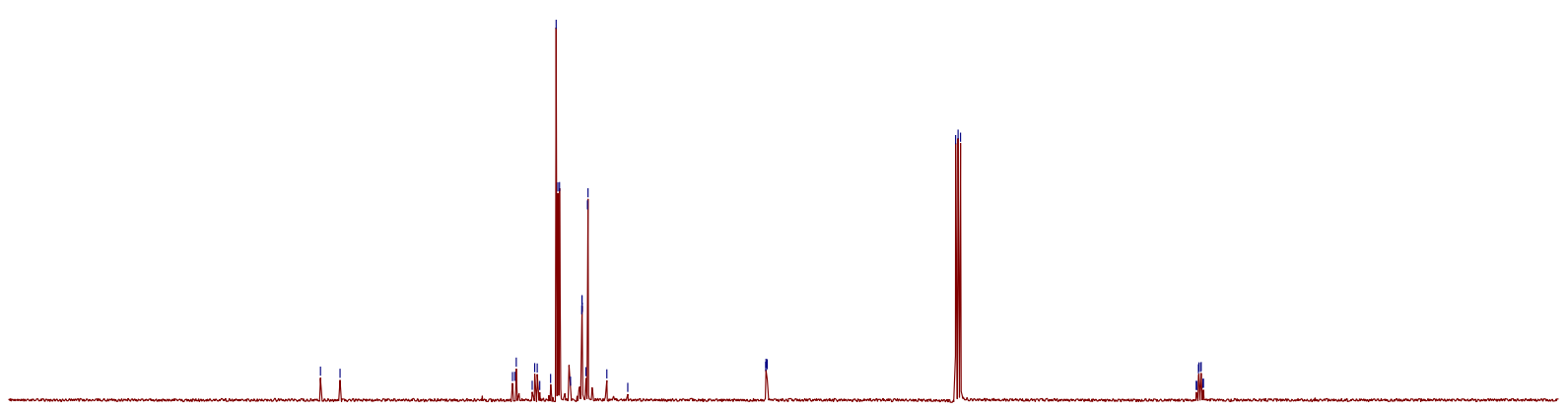


(Z)-2-(1-fluoro-4,4,4-trifluoro-3-phenylbut-1-en-1-yl)naphthalene (7c)<smiles>FC(=CC(c1ccccc1)C(F)(F)F)c1ccc2ccccc2c1</smiles>
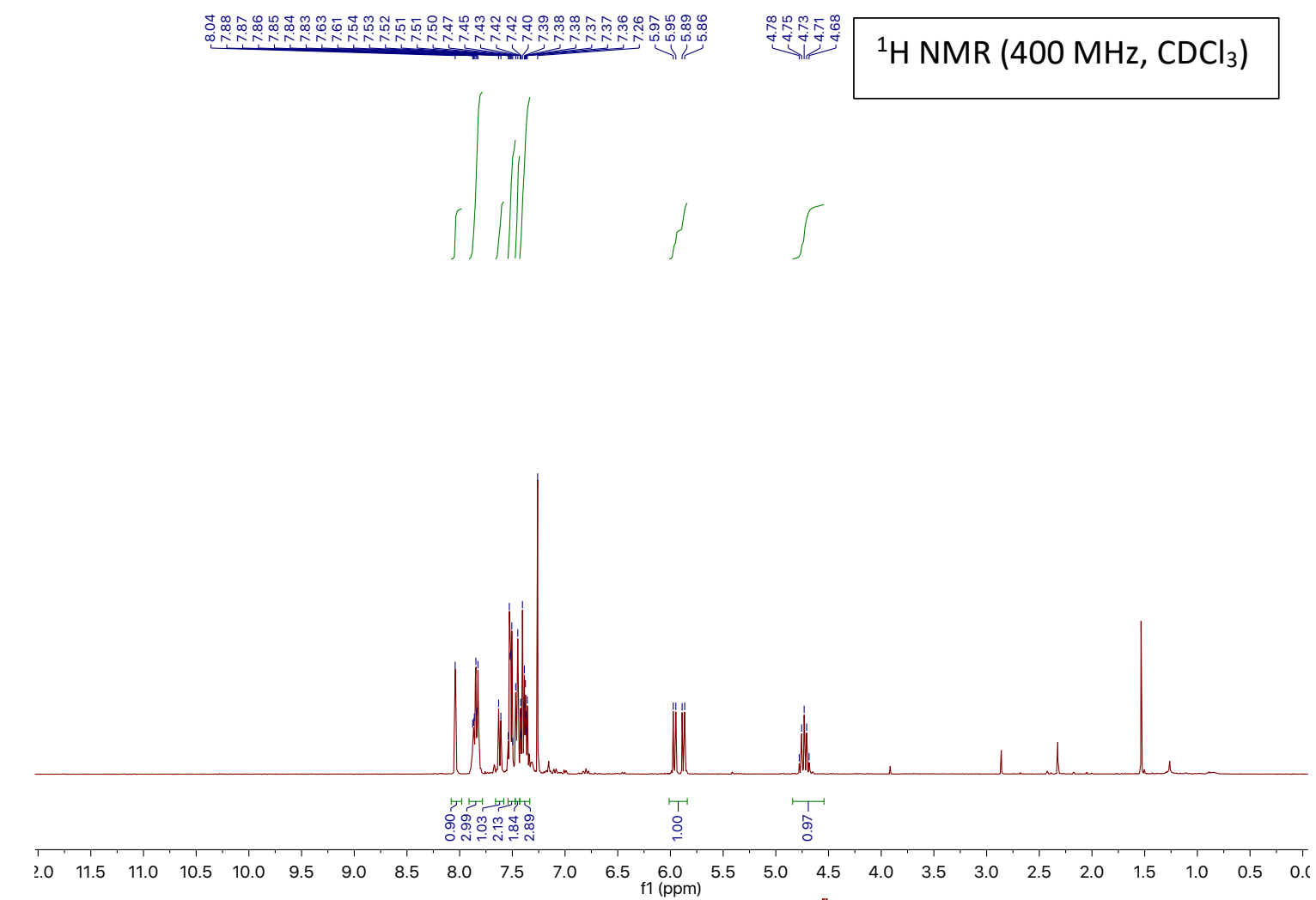

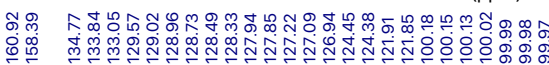

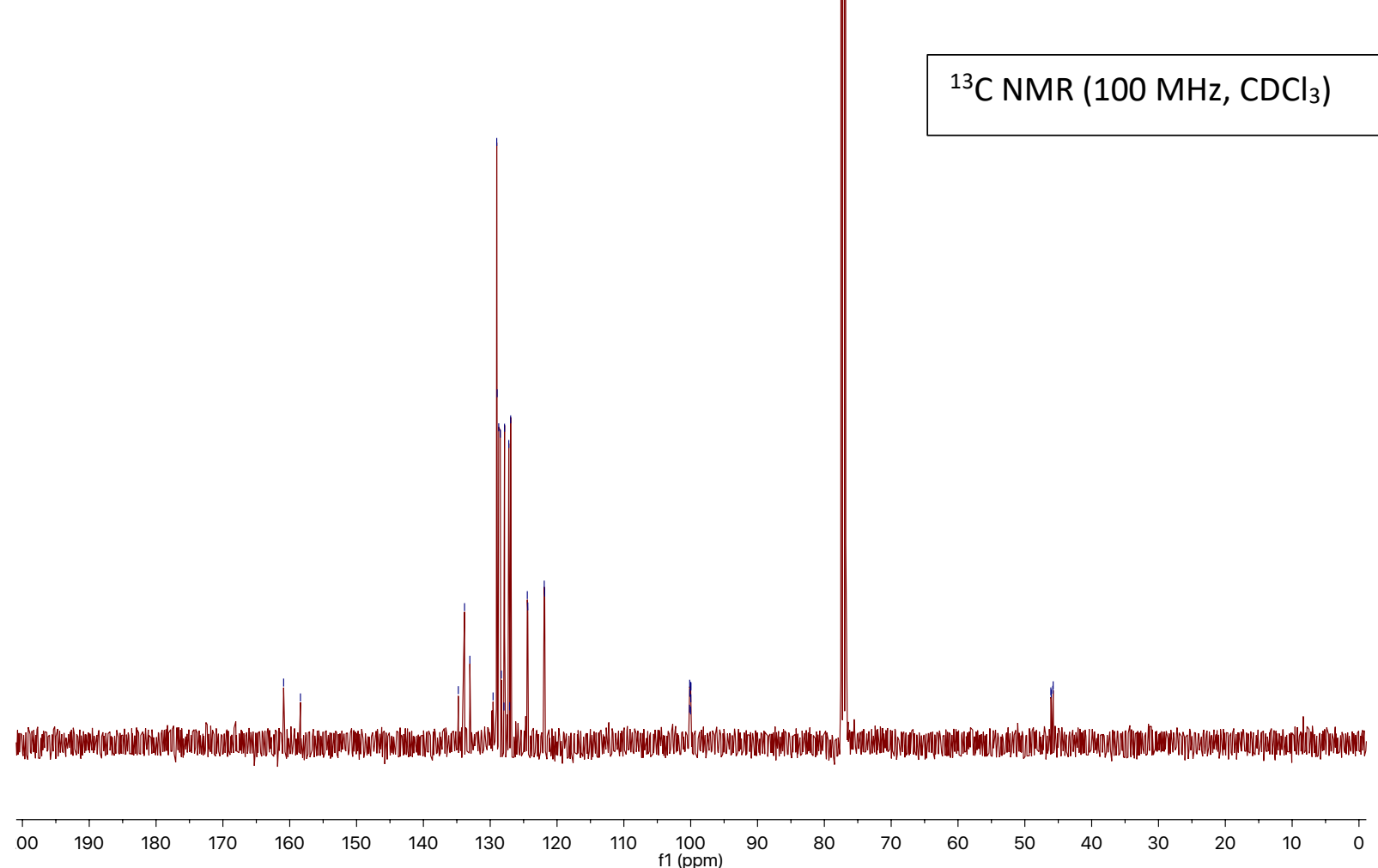


HPLC Chromatograms of allylic chlorides $2 \mathrm{a}, 2 \mathrm{~b}, 2 \mathrm{c}, 2 \mathrm{~d}, 2 \mathrm{e}, 2 \mathrm{~m}$ and $2 \mathrm{~g}$

(E)-(1-chloro-4,4,4-trifluorobut-2-ene-1,3-diyl)dibenzene (2a)

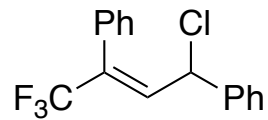
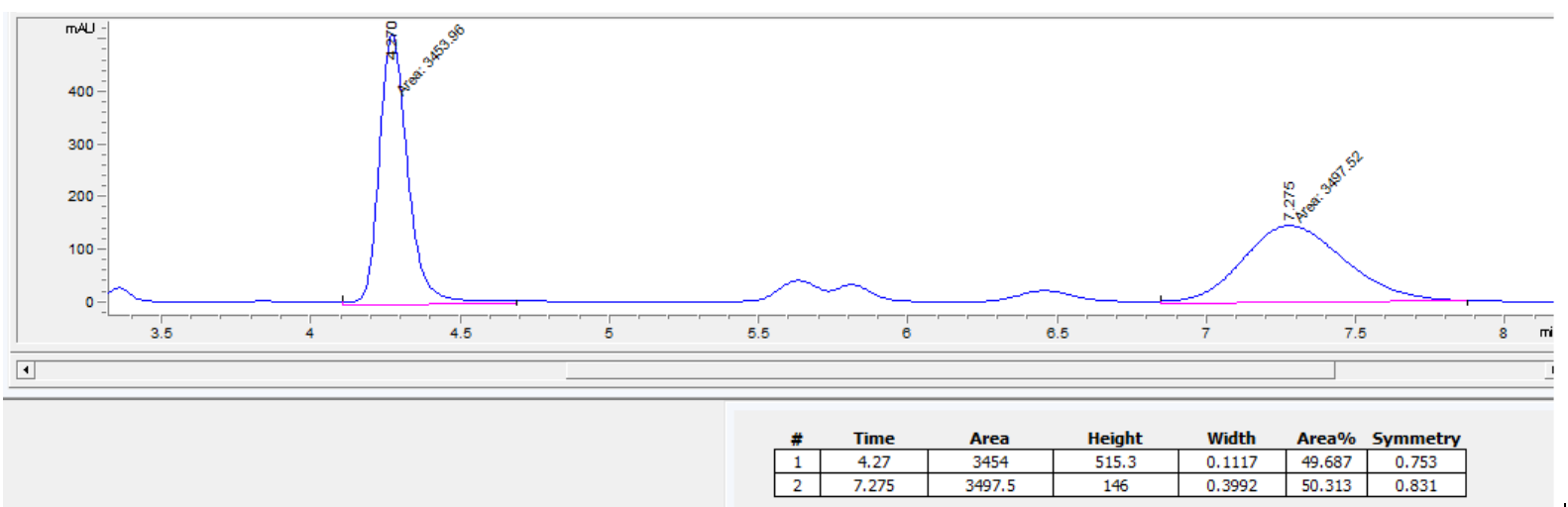

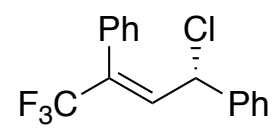

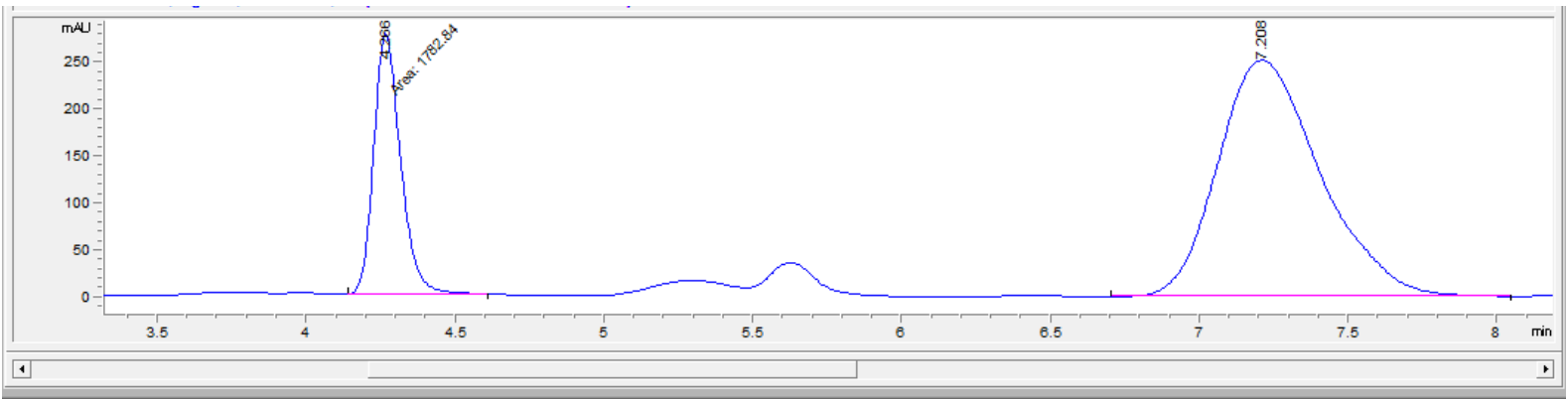

\begin{tabular}{|c|c|c|c|c|c|c|}
\hline 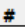 & Time & Area & Height & Width & Area\% & Symmetry \\
\hline 1 & 4.266 & 1782.8 & 279.3 & 0.1064 & 22.873 & 0.759 \\
\hline 2 & 7.208 & 6011.6 & 251.2 & 0.3712 & 77.127 & 0.714 \\
\hline
\end{tabular}

(E)-1-Bromo-4-(1-chloro-4,4,4-trifluoro-3-phenylbut-2-en-1-yl)benzene (2b)

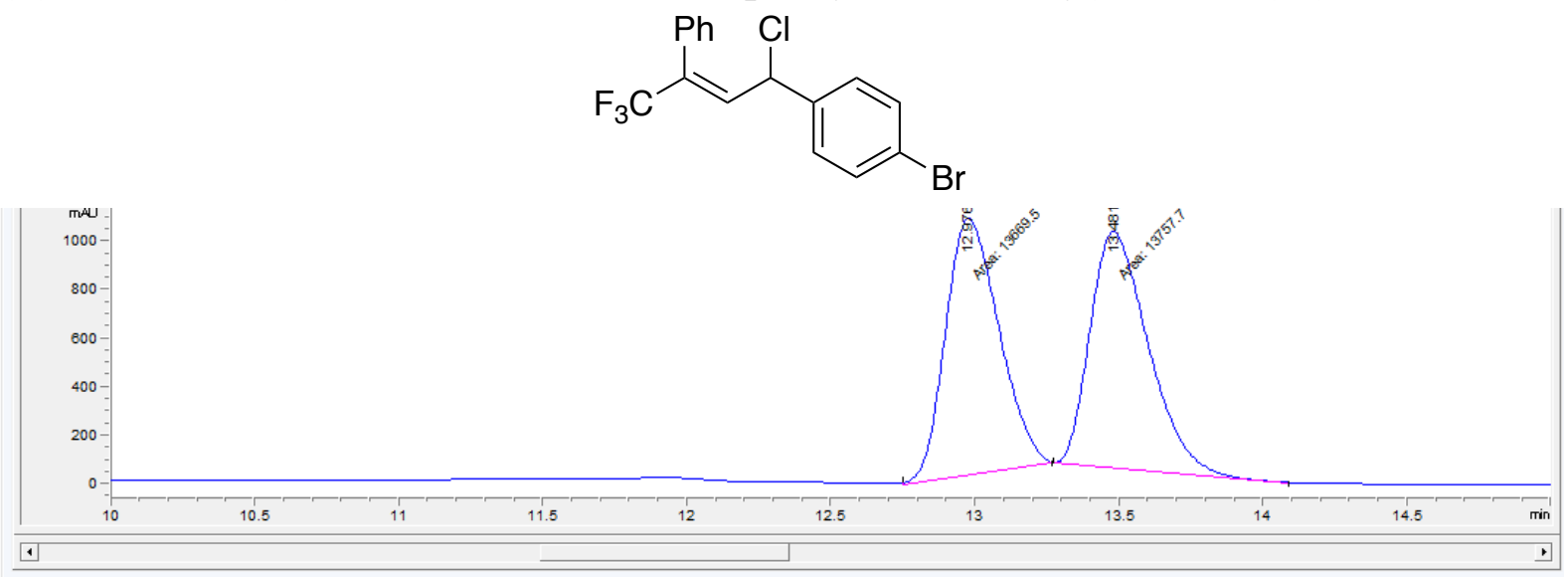


<smiles>FC(F)(F)C(=C[C@H](Cl)c1ccc(Br)cc1)c1ccccc1</smiles>

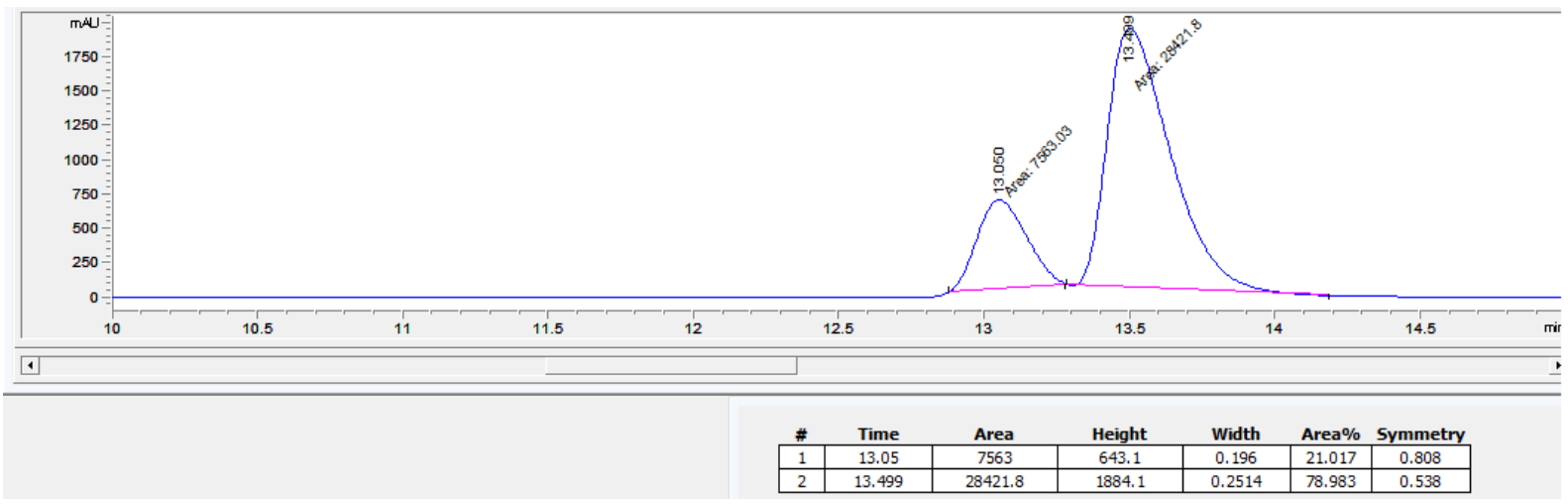

(E)-1-(1-chloro-4,4,4-trifluoro-3-phenylbut-2-en-1-yl)-4-(trifluoromethyl)benzene (2c)<smiles>FC(F)(F)C(=CC(Cl)c1ccc(C(F)(F)F)cc1)c1ccccc1</smiles>

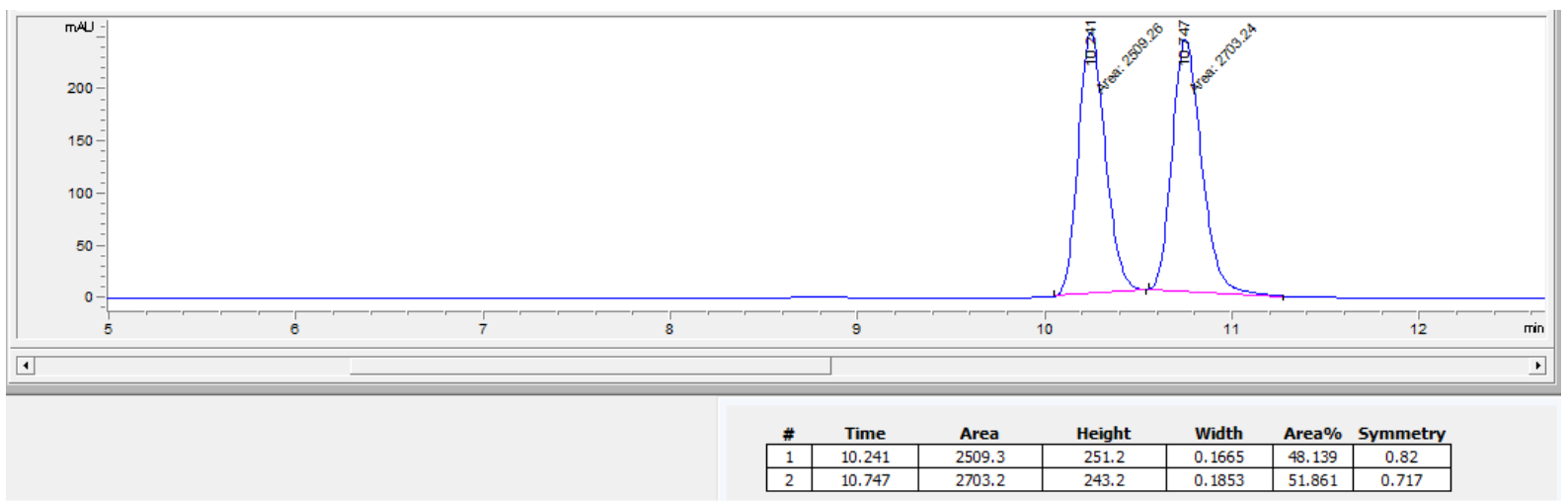<smiles>FC(F)(F)C(=C[C@H](Cl)c1ccc(C(F)(F)F)cc1)c1ccccc1</smiles>

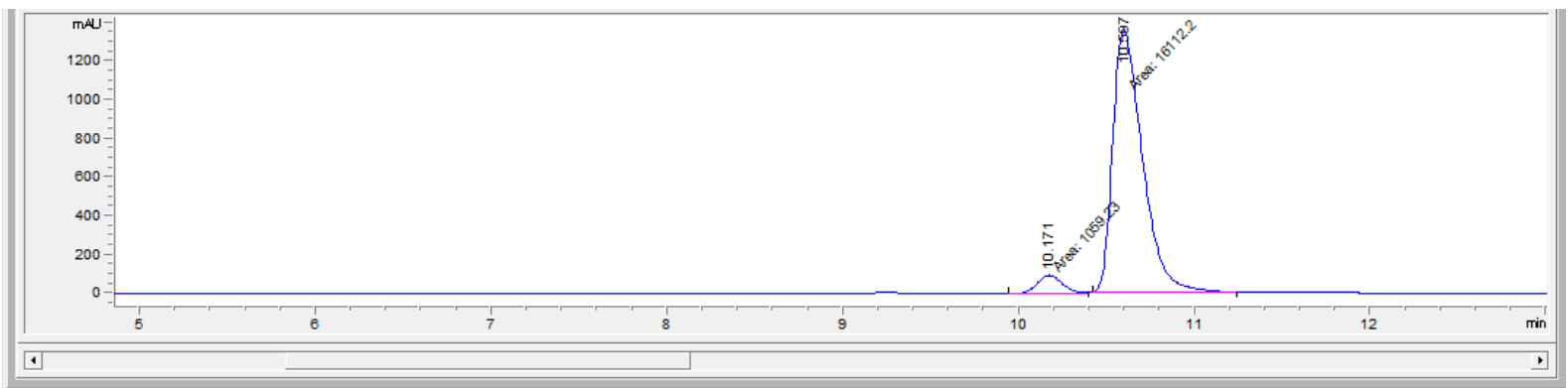

\begin{tabular}{|c|c|c|c|c|c|c|} 
\# & \multicolumn{1}{c}{ Time } & Area & Height & \multicolumn{1}{c|}{ Width } & \multicolumn{2}{c|}{ Area\% } \\
\hline 1 & 10.171 & 1059.2 & 98.4 & 0.1794 & 6.169 & 0.974 \\
\hline 2 & 10.597 & 16112.2 & 1361.3 & 0.1973 & 93.831 & 0.606 \\
\hline
\end{tabular}


(E)-4-(1-chloro-4,4,4-trifluoro-3-phenylbut-2-en-1-yl)benzonitrile (2d)<smiles>N#Cc1ccc(C(Cl)/C=C(\c2ccccc2)C(F)(F)F)cc1</smiles>
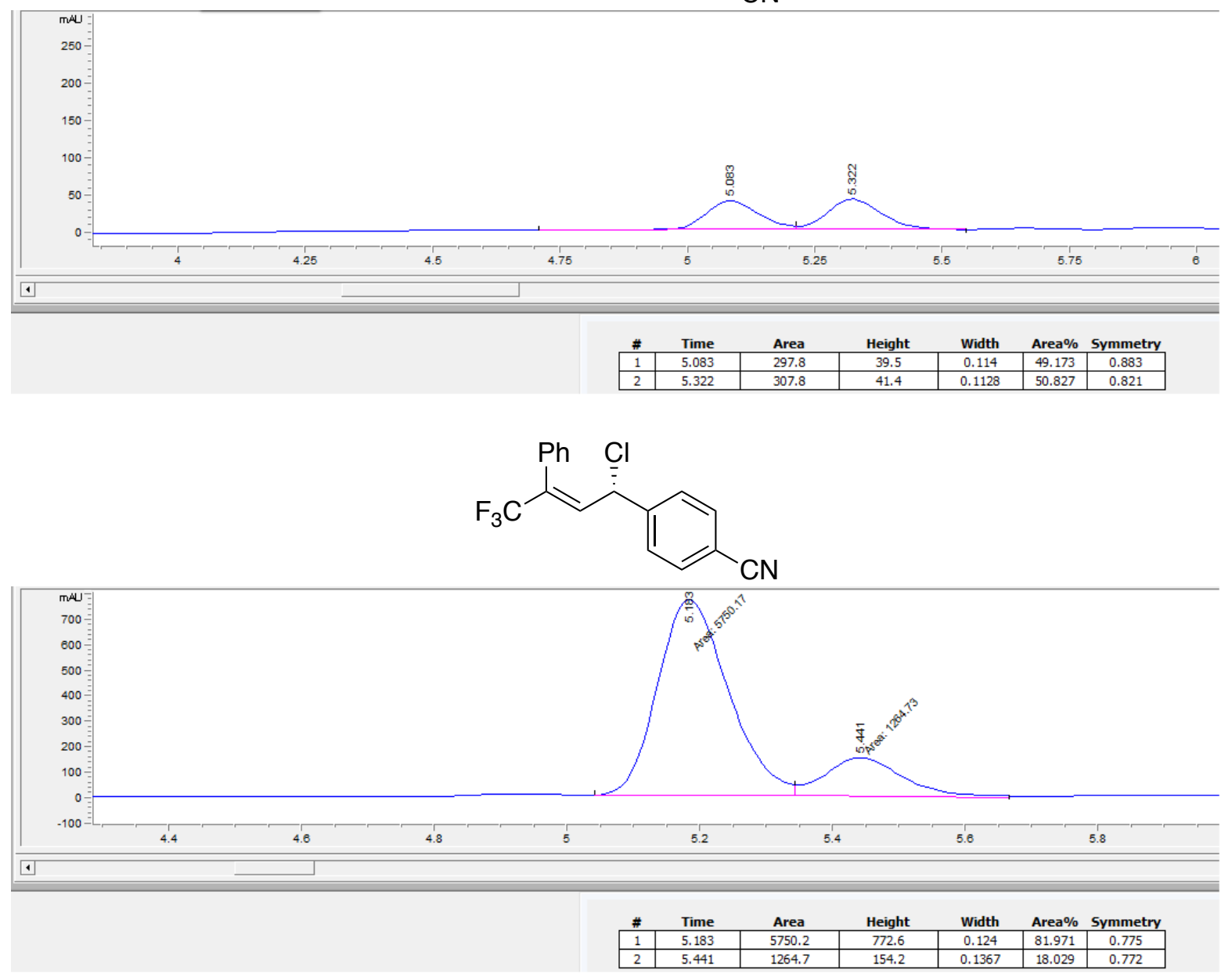

(E)-1-(1-chloro-4,4,4-trifluoro-3-phenylbut-2-en-1-yl)-4-(methylsulfonyl)benzene (2e)<smiles>CO[SH](C)(=O)c1ccc(C(Cl)/C=C(\c2ccccc2)C(F)(F)F)cc1</smiles>

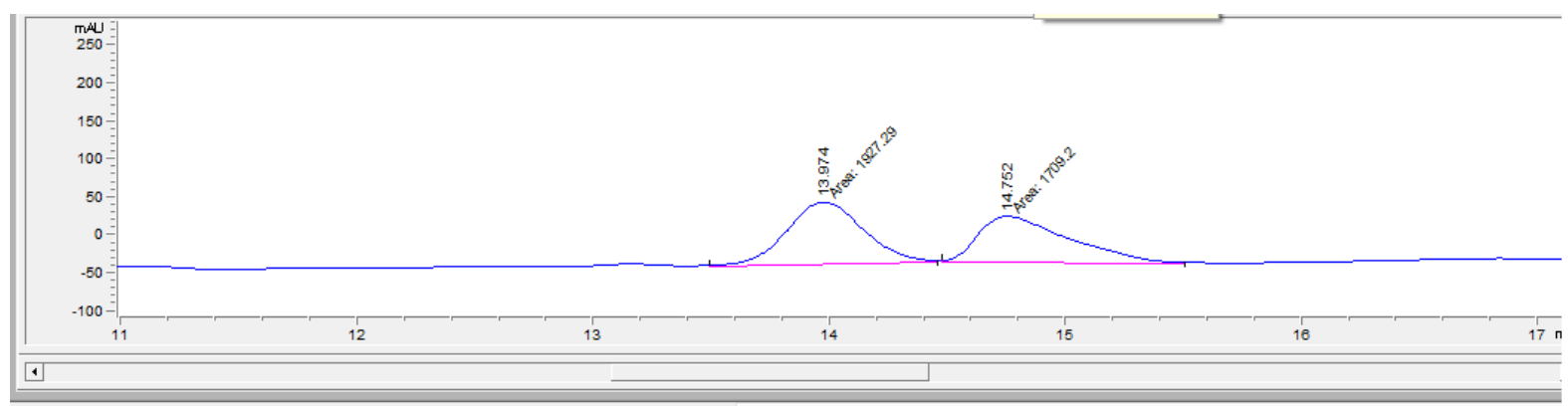

\begin{tabular}{|c|c|c|c|c|c|c|}
\multicolumn{2}{c}{ \# } & \multicolumn{1}{c}{ Time } & Area & \multicolumn{1}{c}{ Height } & Width & \multicolumn{2}{c|}{ Area\% } & \multicolumn{1}{c|}{ Symmetry } \\
\hline 1 & 13.974 & 1927.3 & 83 & 0.387 & 52.999 & 0.906 \\
\hline 2 & 14.752 & 1709.2 & 61.6 & 0.4627 & 47.001 & 0.441 \\
\hline
\end{tabular}

S47 


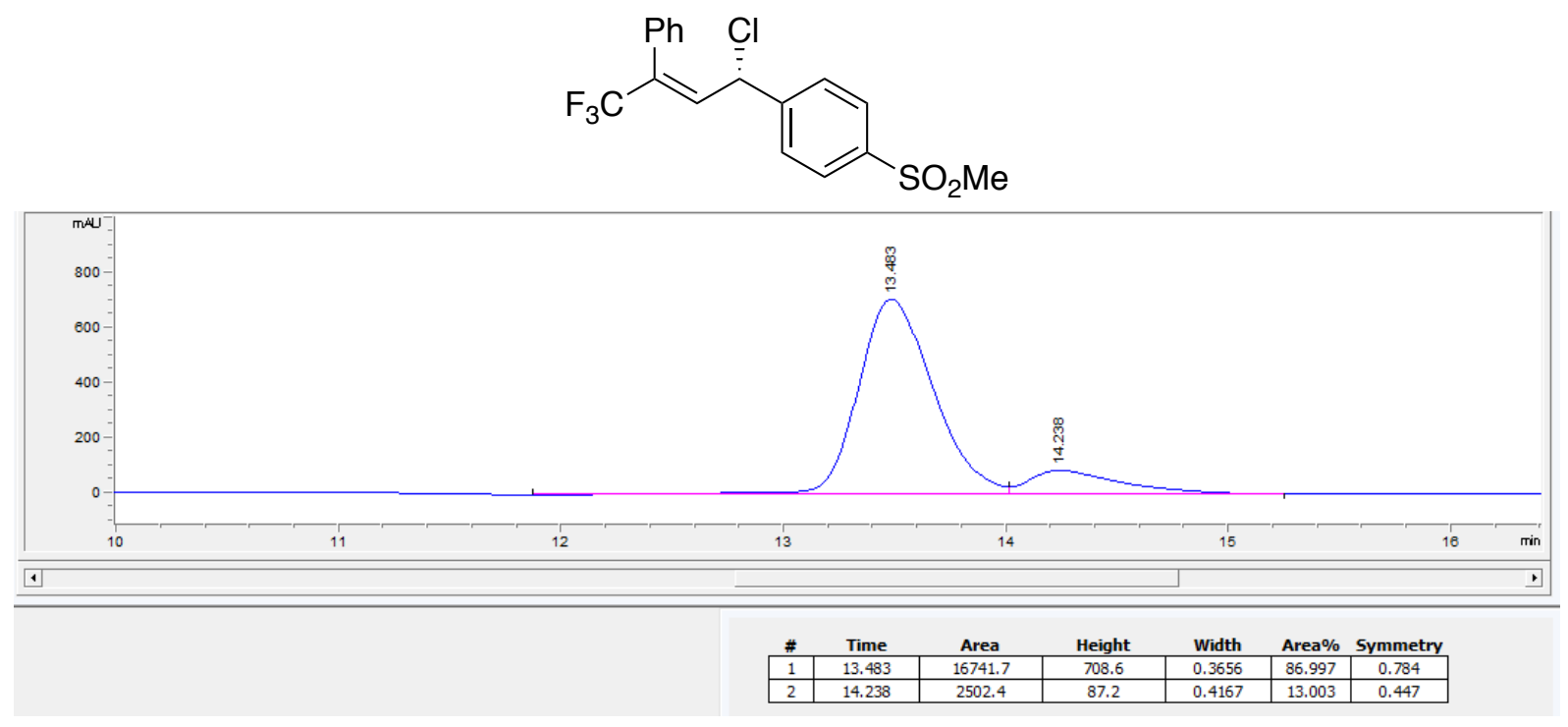

(E)-2-(1-chloro-4,4,4-trifluoro-3-phenylbut-2-en-1-yl)naphthalene (2g)<smiles>FC(F)(F)C(=CC(Cl)c1ccc2ccccc2c1)c1ccccc1</smiles>
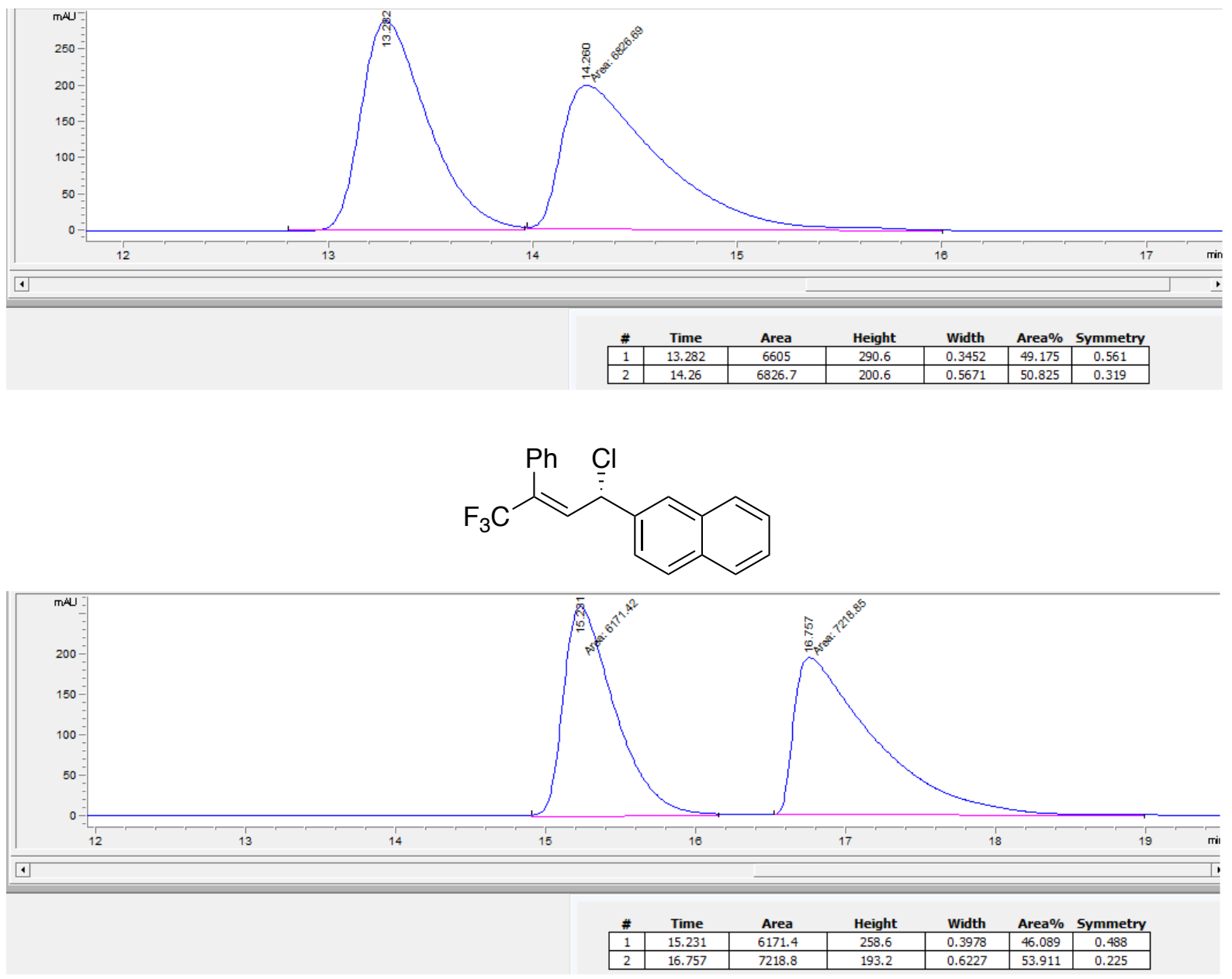

S48 
(E)-(1-chloro-4,4,4-trifluoro-3-methylbut-2-en-1-yl)benzene (2k)
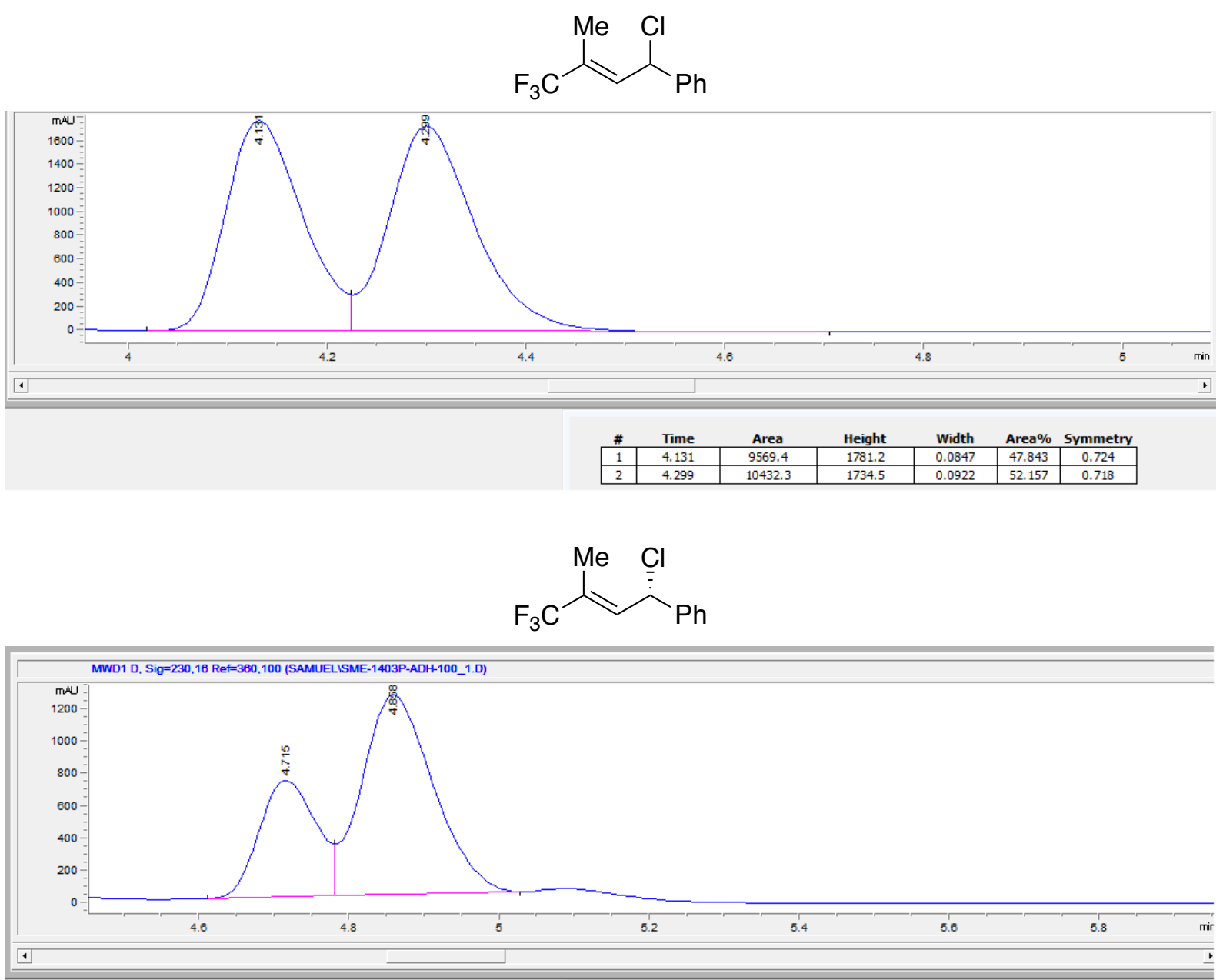

\begin{tabular}{|c|c|c|c|c|c|c|}
\hline \# & Time & Area & Height & Width & Area $\%$ & Symmetry \\
\hline 1 & 4.715 & 3822.3 & 726.8 & 0.0814 & 31.845 & 0.841 \\
\hline & 858 & 80.3 & 1237.4 & 1011 & 55 & 0.749 \\
\hline
\end{tabular}


HPLC Chromatograms of vinyl chlorides 5a, 5b, 5c, 5d, 5e, 5m and 5g

(Z)-(1-chloro-4,4,4-trifluorobut-1-ene-1,3-diyl)dibenzene (5a)
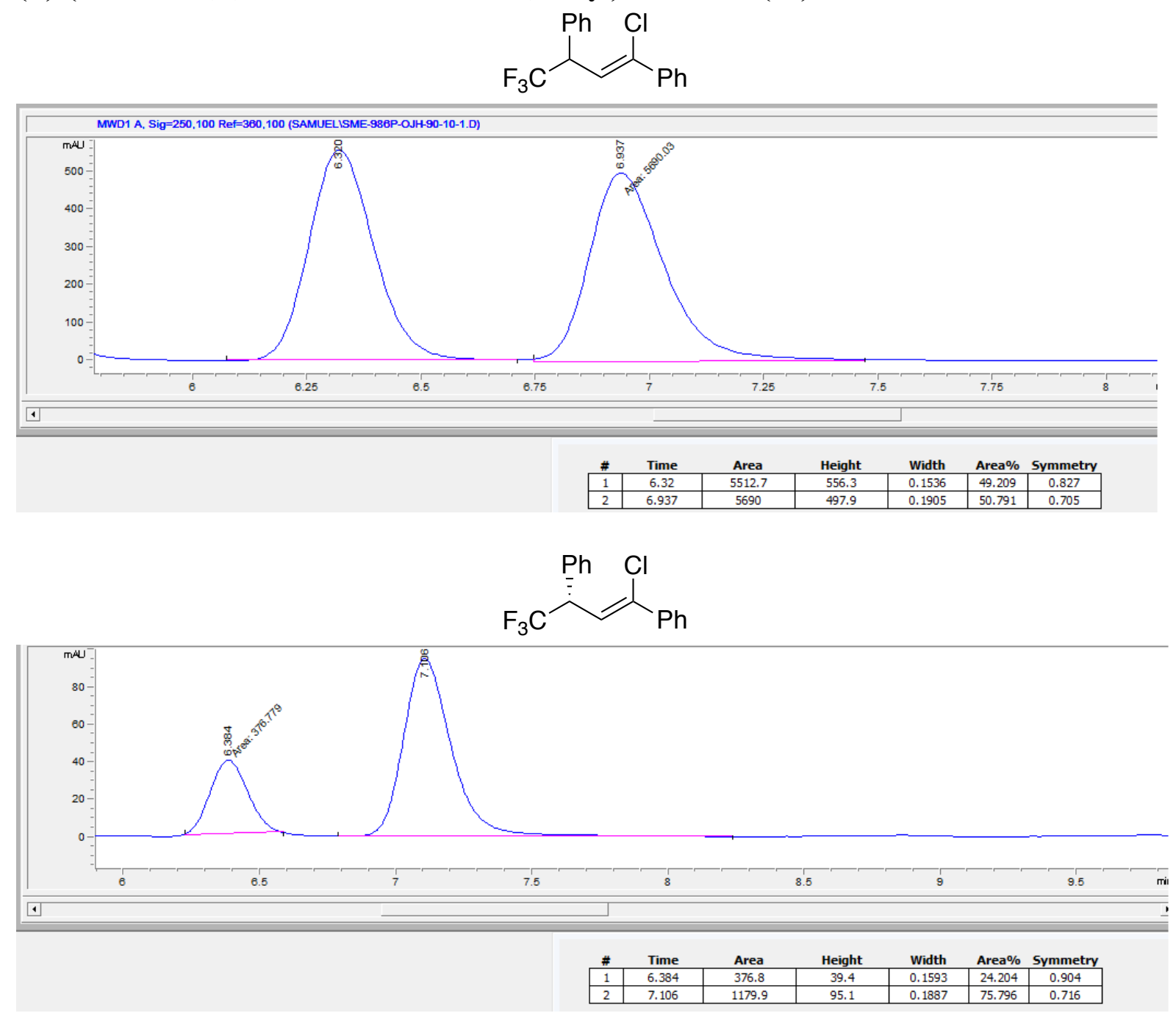

(Z)-1-bromo-4-(1-chloro-4,4,4-trifluoro-3-phenylbut-1-en-1-yl)benzene (5b)<smiles>FC(F)(F)C(/C=C(\Cl)c1ccc(Br)cc1)c1ccccc1</smiles>

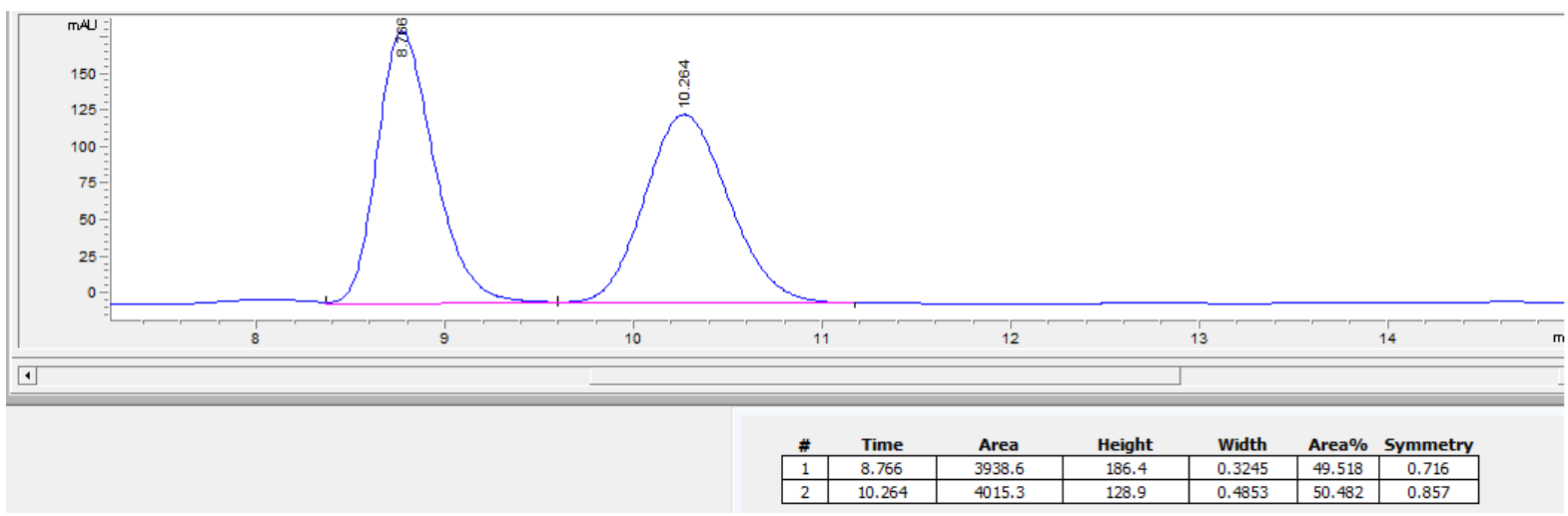



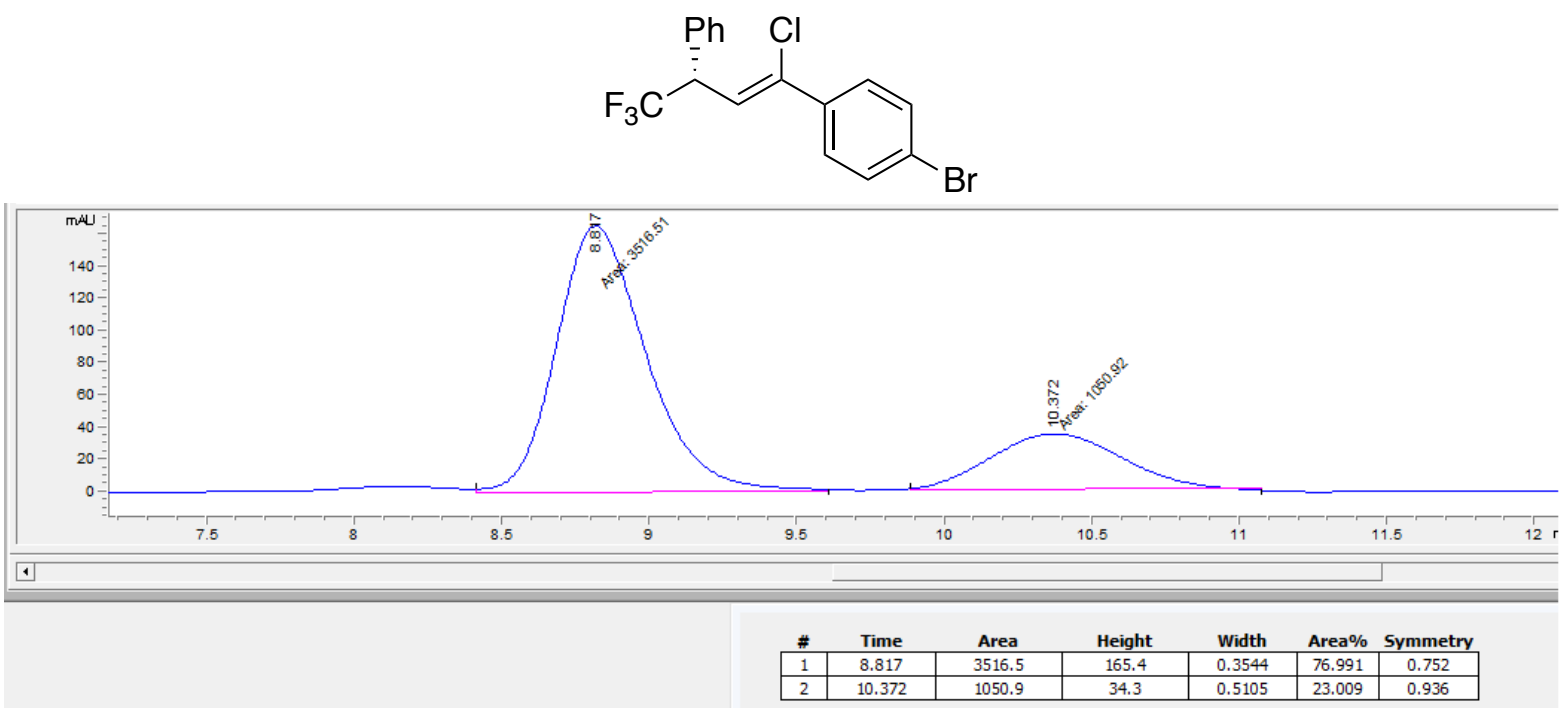

(Z)-1-(1-chloro-4,4,4-trifluoro-3-phenylbut-1-en-1-yl)-4-(trifluoromethyl)benzene (5c)

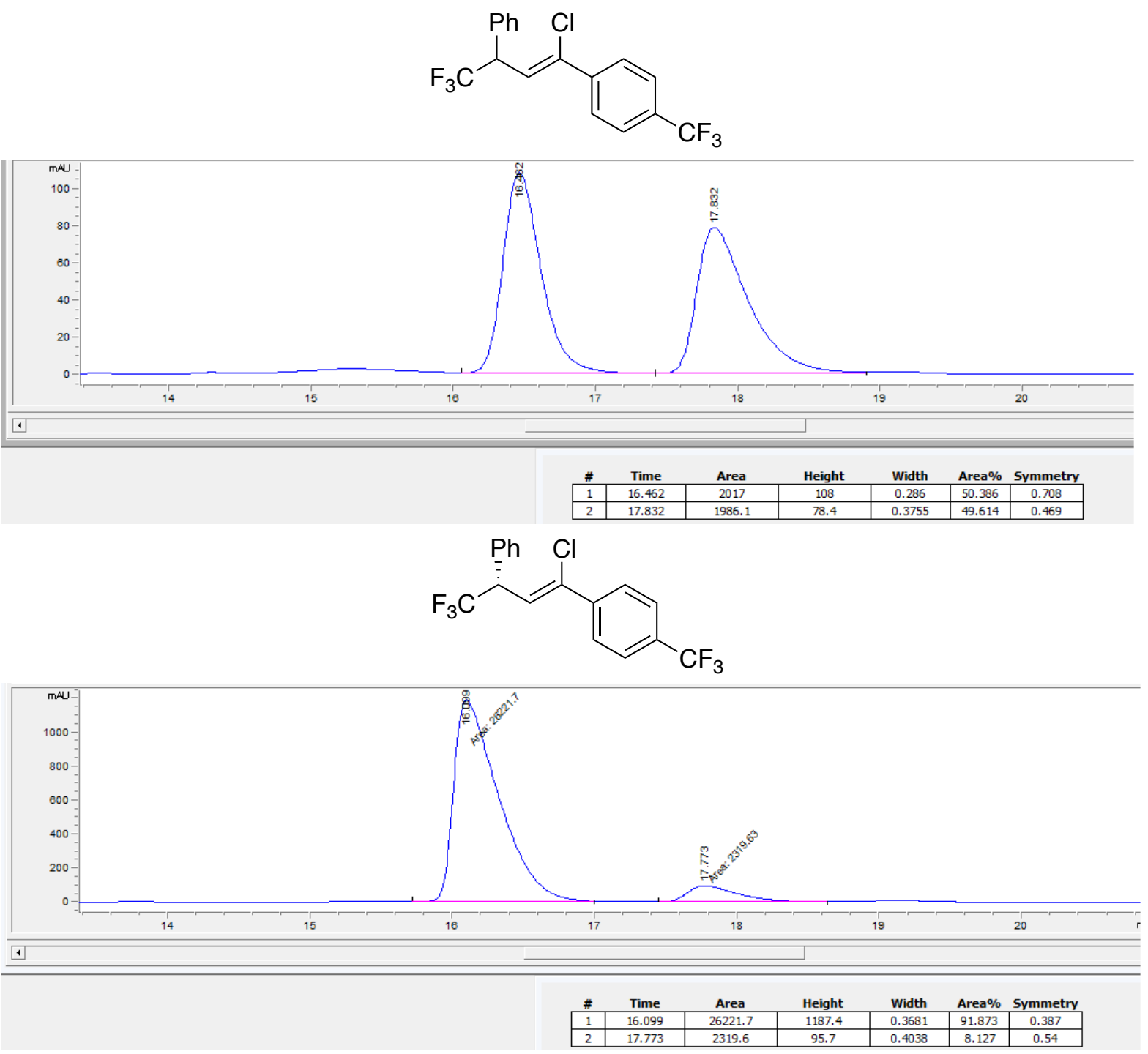




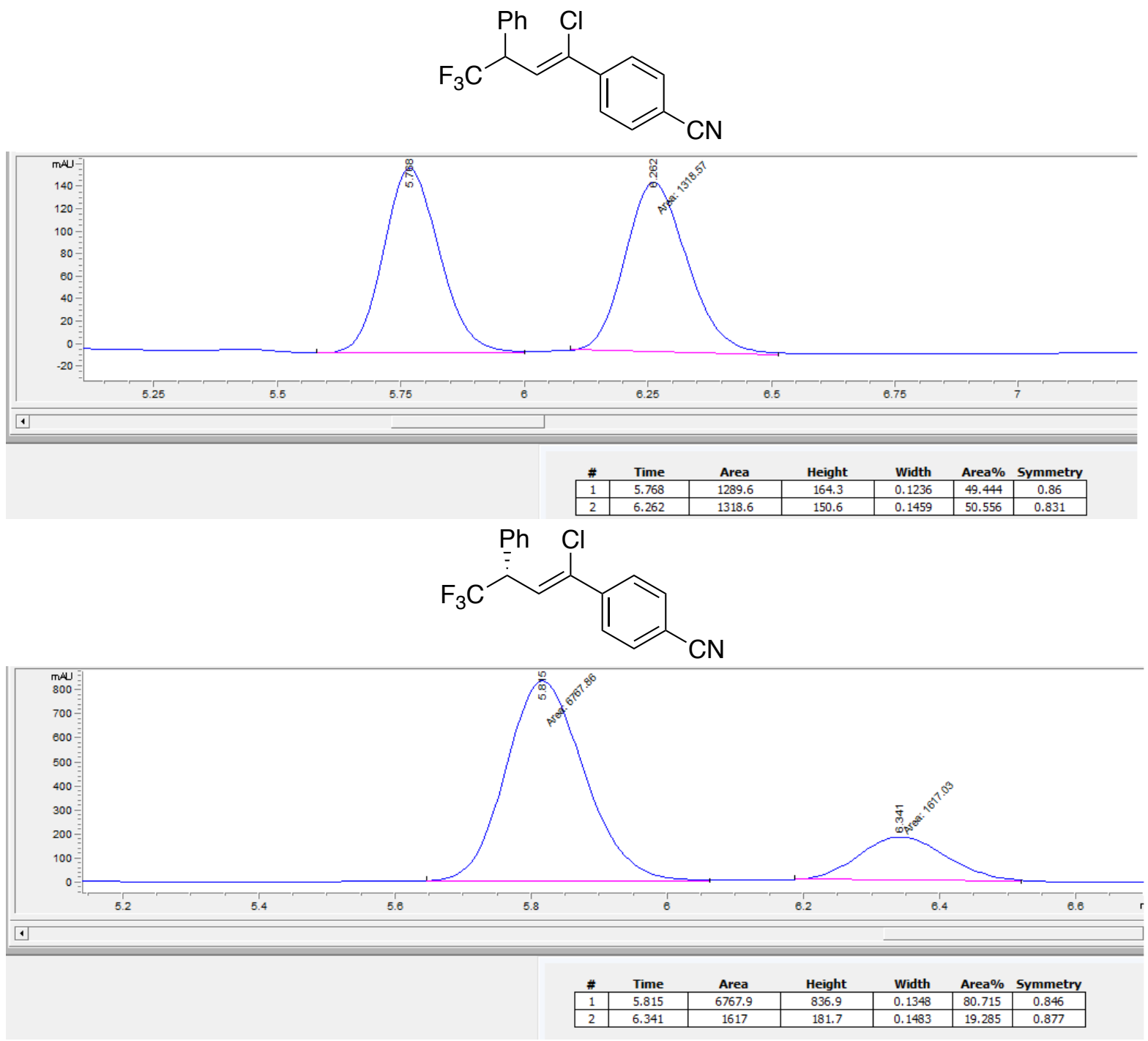

(Z)-1-(1-chloro-4,4,4-trifluoro-3-phenylbut-1-en-1-yl)-4-(methylsulfonyl)benzene (5e)

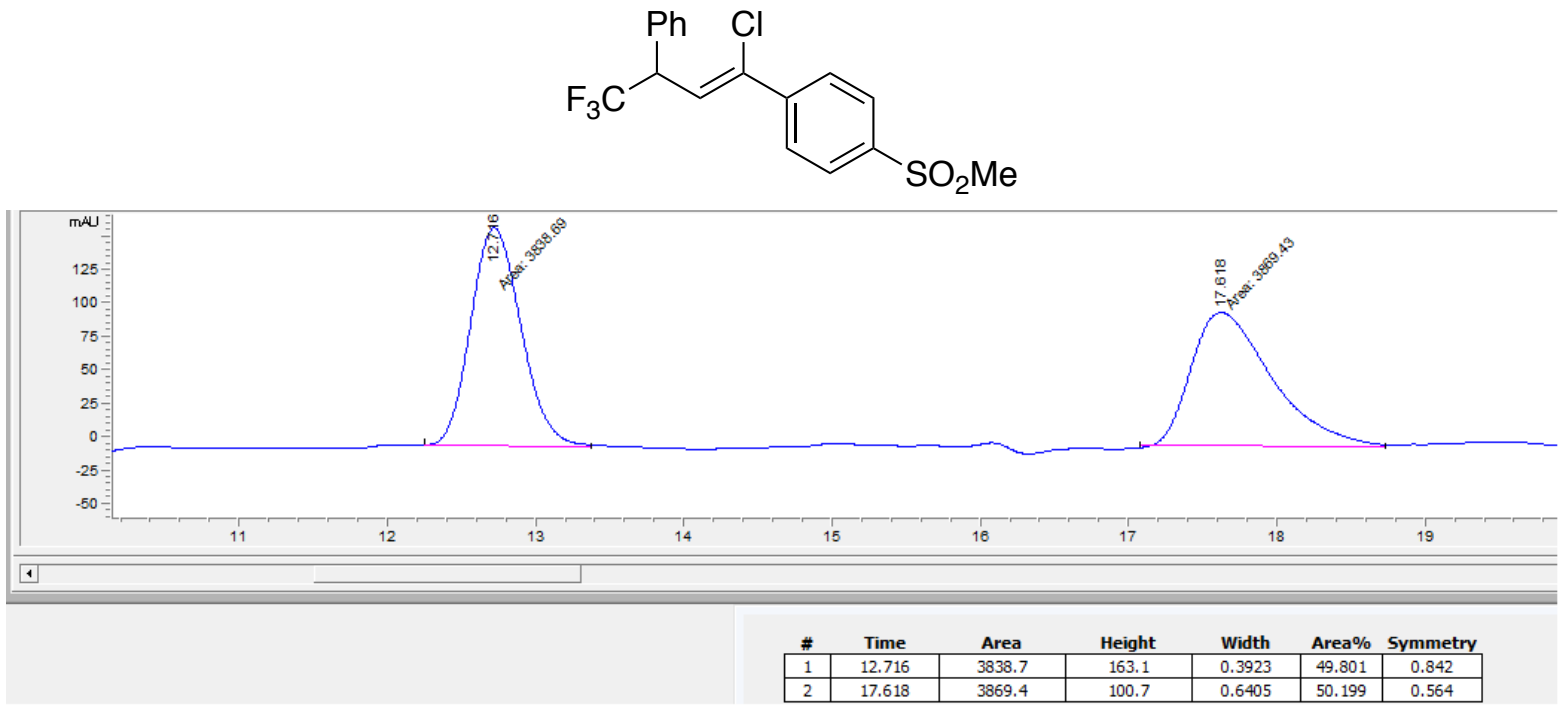


<smiles>CO[SH](C)(=O)c1ccc(/C(Cl)=C/C(c2ccccc2)C(F)(F)F)cc1</smiles>

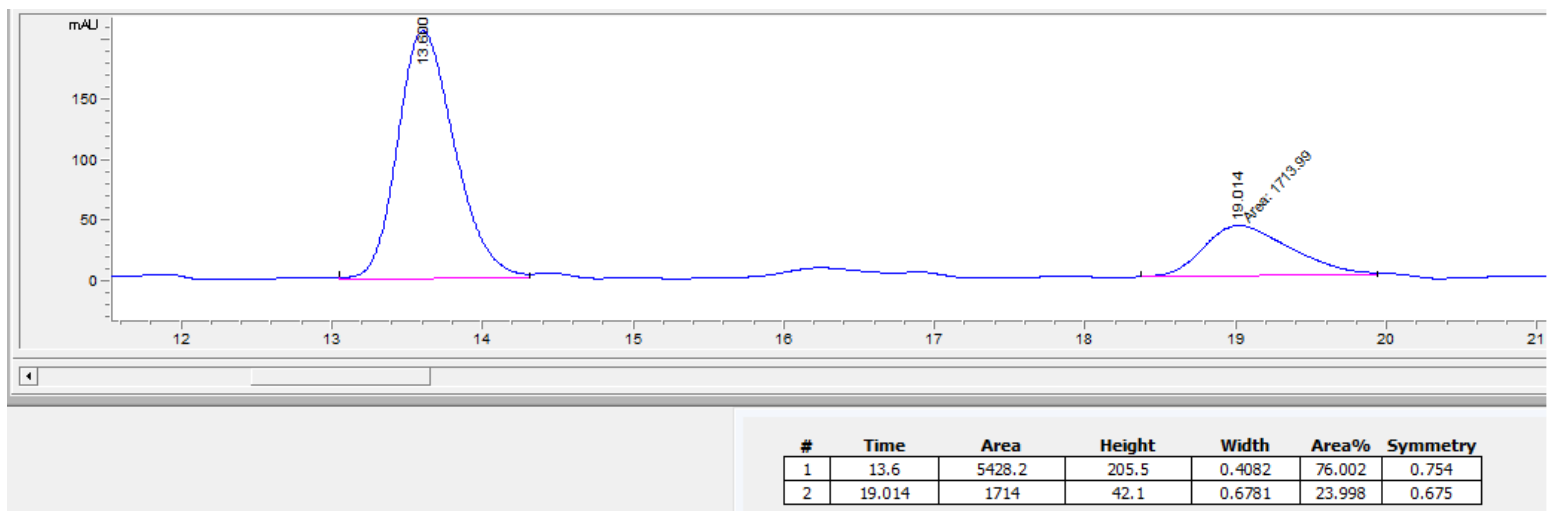

(Z)-2-(1-chloro-4,4,4-trifluoro-3-phenylbut-1-en-1-yl)naphthalene (5g)<smiles>FC(F)(F)C(/C=C(\Cl)c1ccc2ccccc2c1)c1ccccc1</smiles>
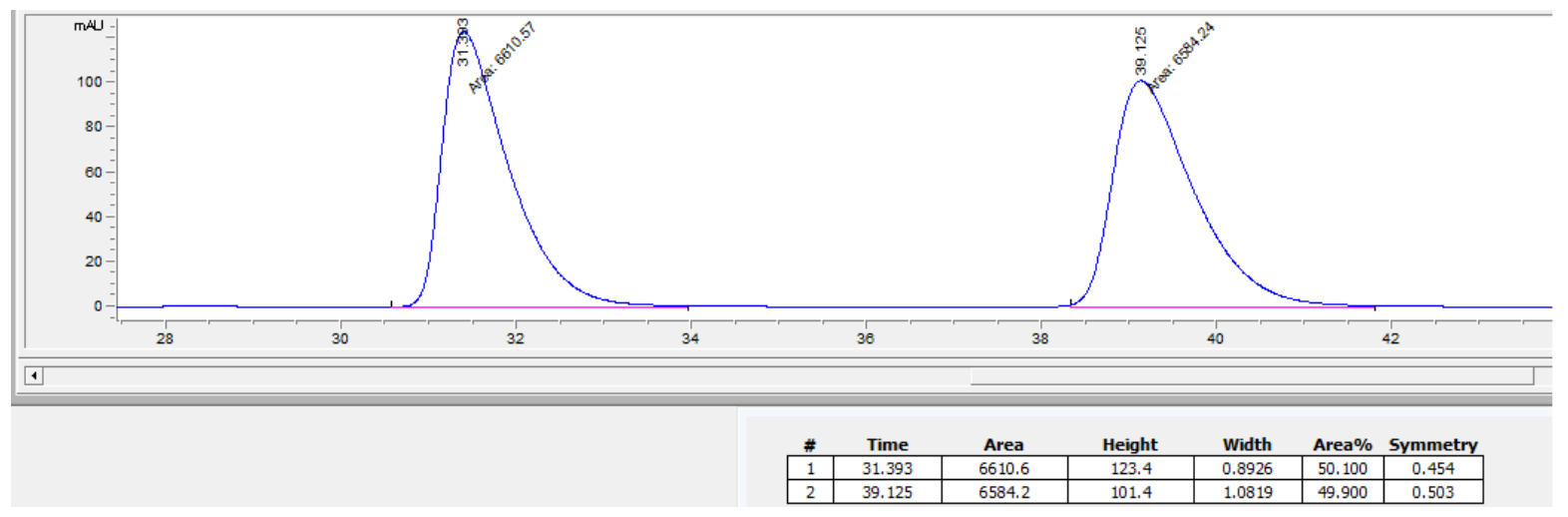<smiles>FC(F)(F)C(/C=C(\Cl)c1ccc2ccccc2c1)c1ccccc1</smiles>
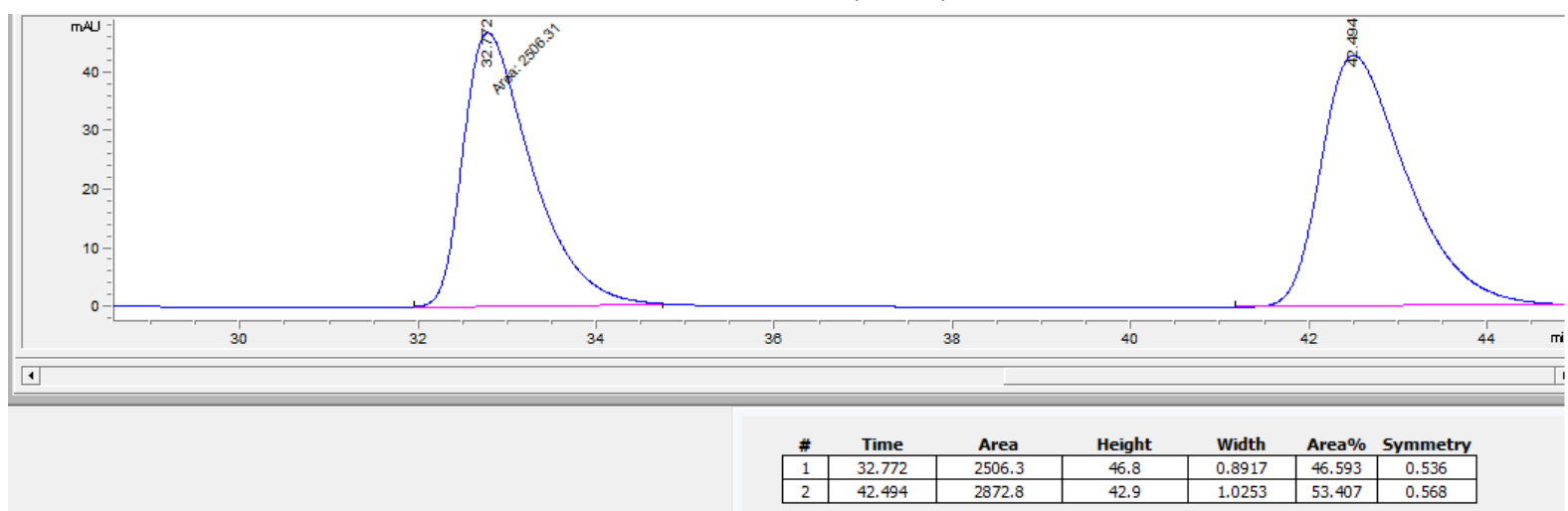

S53 

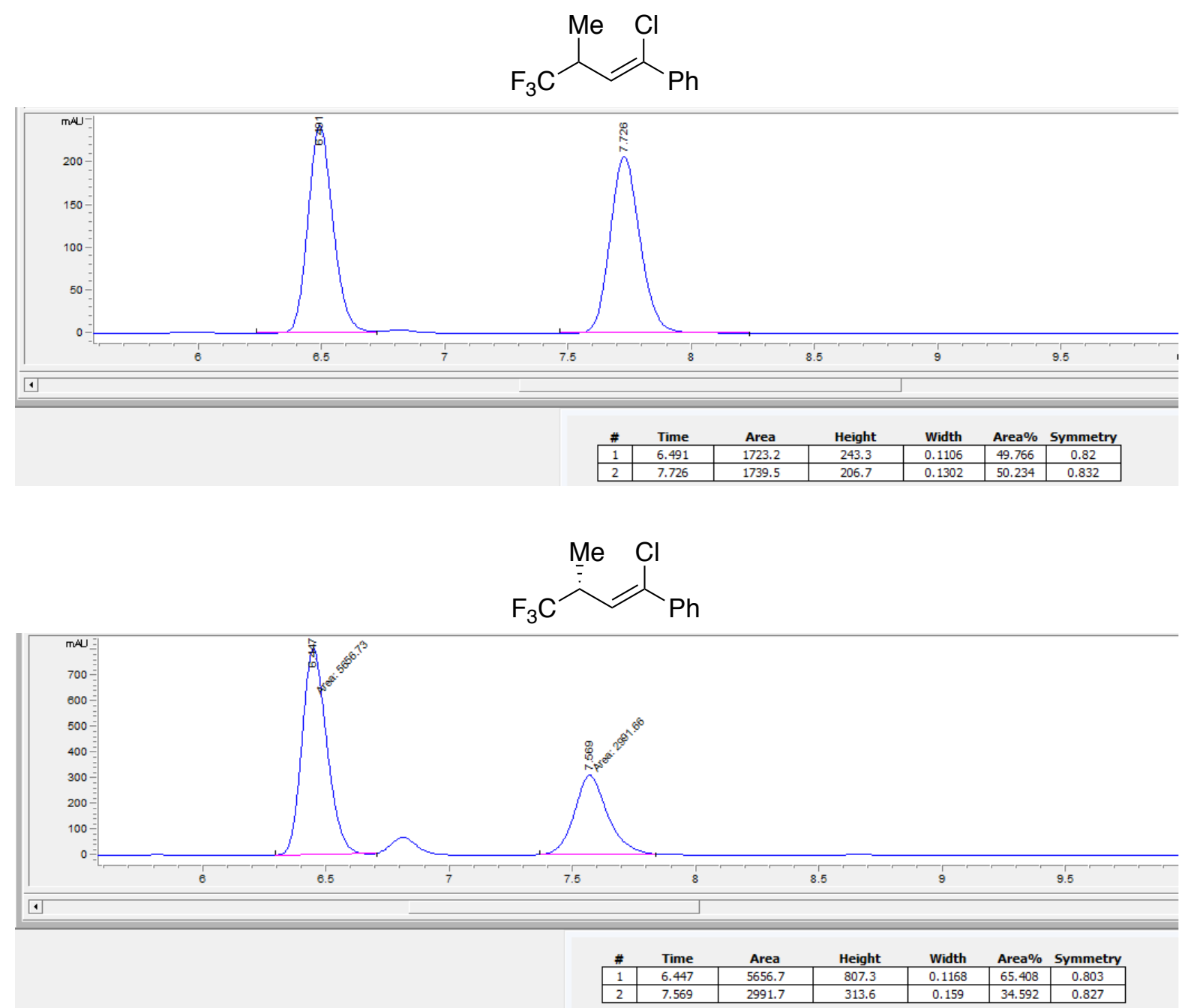